\title{
WestVirginiaUniversity
}

THE RESEARCH REPOSITORY @ WVU

Graduate Theses, Dissertations, and Problem Reports

2006

\section{Models for calculating confidence intervals for neural networks}

Ashutosh R. Nandeshwar

West Virginia University

Follow this and additional works at: https://researchrepository.wvu.edu/etd

\section{Recommended Citation}

Nandeshwar, Ashutosh R., "Models for calculating confidence intervals for neural networks" (2006). Graduate Theses, Dissertations, and Problem Reports. 1716.

https://researchrepository.wvu.edu/etd/1716

This Thesis is protected by copyright and/or related rights. It has been brought to you by the The Research Repository @ WVU with permission from the rights-holder(s). You are free to use this Thesis in any way that is permitted by the copyright and related rights legislation that applies to your use. For other uses you must obtain permission from the rights-holder(s) directly, unless additional rights are indicated by a Creative Commons license in the record and/ or on the work itself. This Thesis has been accepted for inclusion in WVU Graduate Theses, Dissertations, and Problem Reports collection by an authorized administrator of The Research Repository @ WVU. For more information, please contact researchrepository@mail.wvu.edu. 


\title{
Models for Calculating Confidence Intervals for Neural Networks
}

\author{
Ashutosh R. Nandeshwar
}

\author{
Thesis submitted to the \\ College of Engineering and Mineral Resources \\ at West Virginia University \\ in partial fulfillment of the requirements \\ for the degree of
}

\section{Master of Science \\ in}

Industrial Engineering

Robert C. Creese, Ph.D., (Chair)

Shahab D. Mohaghegh, Ph.D., Bhaskaran Gopalakrishnan, Ph.D.

Department of Industrial and Management Systems Engineering

Morgantown, West Virginia 


\title{
Models for Calculating Confidence Intervals for Neural Networks
}

\author{
Ashutosh R. Nandeshwar
}

\begin{abstract}
This research focused on coding and analyzing existing models to calculate confidence intervals on the results of neural networks. The three techniques for determining confidence intervals determination were the non-linear regression, the bootstrapping estimation, and the maximum likelihood estimation. Confidence intervals for non-linear regression, bootstrap estimation, and maximum likelihood were coded in Visual Basic. The neural network used the backpropagation algorithm with an input layer, one hidden layer and an output layer with one unit. The hidden layer had a logistic or binary sigmoidal activation function and the output layer had a linear activation function. These techniques were tested on various data sets with and without additional noise. Out of eight cases studied, non-linear regression and bootstrapping each had the four lowest values for the average coverage probability minus the nominal probability. For the average coverage probabilities minus the nominal probabilities of all data sets, the bootstrapping estimation obtained the lowest values. The ranges and standard deviations of the coverage probabilities over 15 simulations for the three techniques were computed, and it was observed that the non-linear regression obtained consistent results with the least range and standard deviation, and bootstrapping had the largest ranges and standard deviations. The bootstrapping estimation technique gave a slightly better average coverage probability (CP) minus nominal values than the non-linear regression method, but it had considerably more variation in individual simulations. The maximum likelihood estimation had the poorest results with respect to the average $\mathrm{CP}$ minus nominal values.
\end{abstract}




\section{Acknowledgments}

I would like to express my sincere gratitude towards Dr. R. C. Creese for selecting me as his graduate research assistant and for his constant guidance in everything. He has been a great inspiration and helped me to see the things with a total different perspective. I don't think it would have been possible to complete my masters this effectively had I not worked under his supervision. Thanks Dr. Creese.

I would also like to thank Dr. S. D. Mohaghegh, for his excellent idea of this research, and his constant support and guidance. His keen interest in this topic has made this thesis possible. I would like to extend my thanks towards Dr. B. Gopalakrishnan for his time and superb support throughout my masters.

I am especially thankful to Dr. R. Chandran of the Plant and Social Sciences Department for his constant motivation and guidance on both professional and personal fronts. I am also thankful to all of my friends here in the U.S.A and in India for showing me the correct path through tough times.

Above all, I would like to thank my parents and family for everything. Words cannot suffice to express what they have done for me. 


\section{Table of Contents}

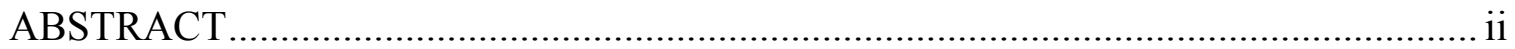

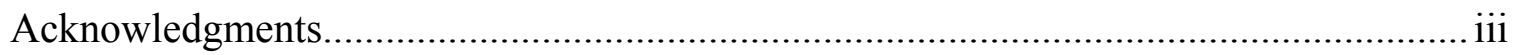

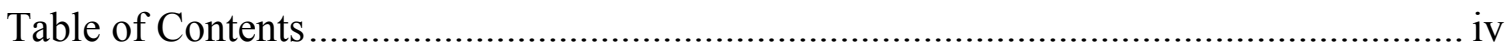

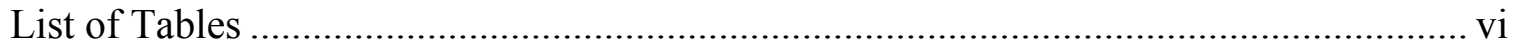

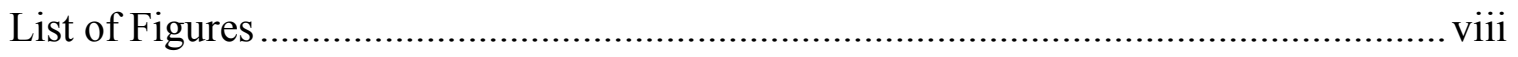

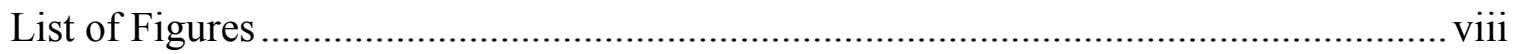

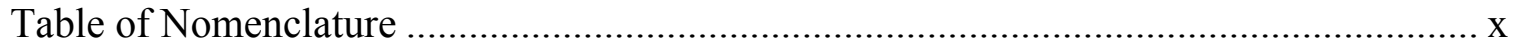

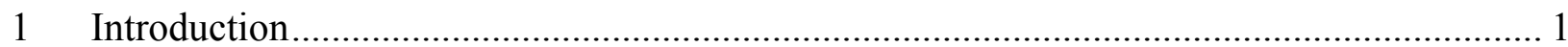

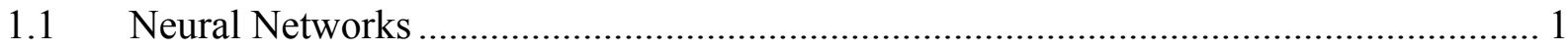

1.1.1 What is an Artificial Neural Network? .......................................................... 1

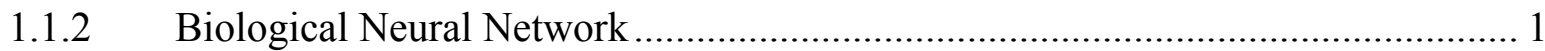

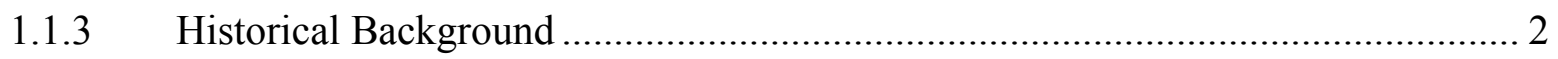

1.1.4 Architecture of a Feed-forward Network ……................................................... 3

1.1.5 Activation Function .................................................................................... 5

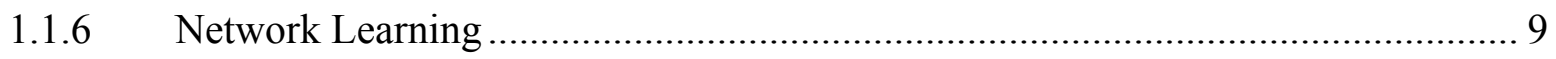

1.1.7 Backpropagation Algorithm....................................................................... 10

1.2 Need of Confidence Intervals for Neural Networks ……………............................... 12

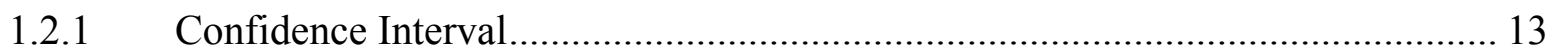

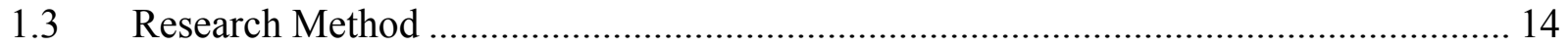

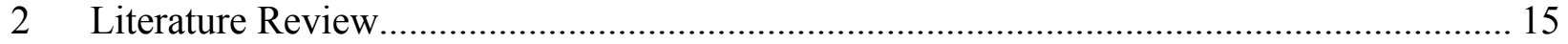

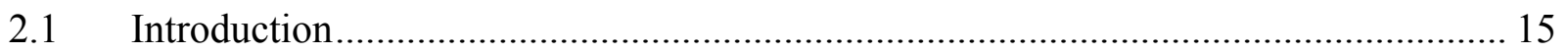

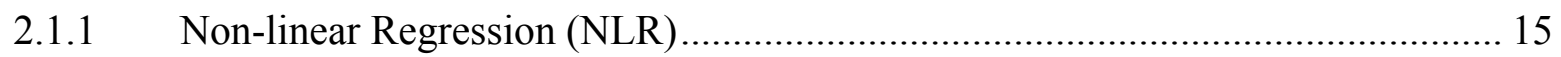

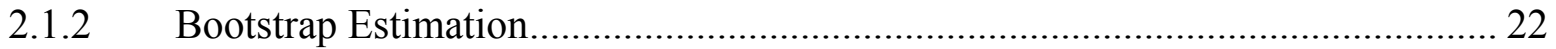

2.1.3 Maximum Likelihood (ML) Estimation …………………………………….... 25

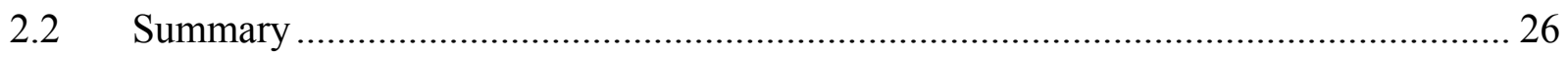

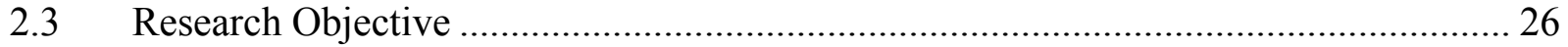

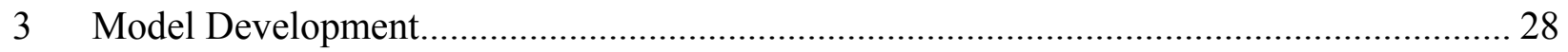

3.1 Development of an Artificial Neural Network Model .............................................. 28

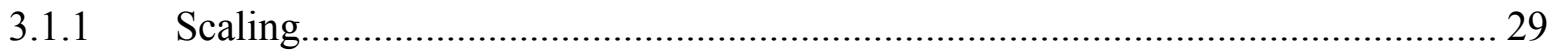

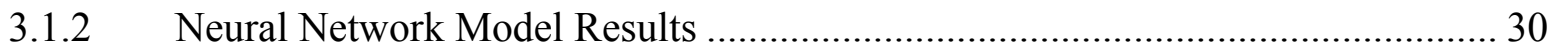


3.1.3 Random Weight Initialization ............................................................ 31

3.2 Development of Confidence Interval for Linear Regression ................................... 34

3.2.1 Linear Regression Confidence Intervals for Tree Data Set [46]........................ 35

3.3 Development of Confidence Interval via Non-linear Regression.............................. 37

3.3.1 Steps for Finding Confidence Intervals Using Non-Linear Regression .............. 39

Validation of the NLR model .................................................................... 39

3.4 Development of Confidence Interval via Bootstrap Estimation ............................... 40

3.4.1 Validation of Bootstrapping Model ............................................................ 40

3.5 Development of Confidence Interval via Maximum-likelihood Estimation (MLE) .... 42

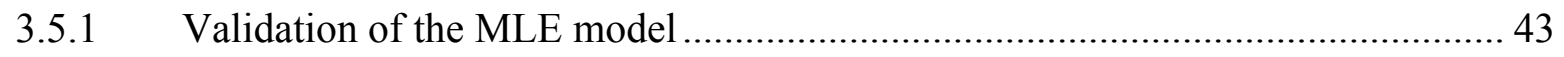

3.6 Comparison of Confidence Intervals for Linear Regression and Neural Networks ..... 43

4 Assessing Confidence Estimation Techniques ............................................................ 49

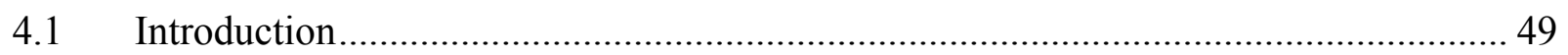

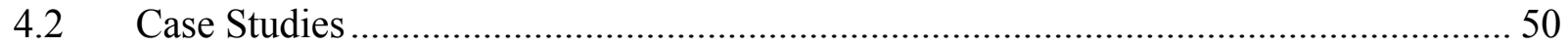

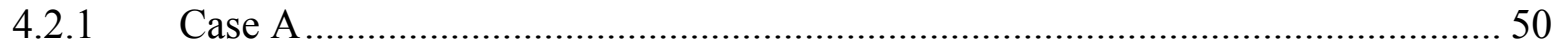

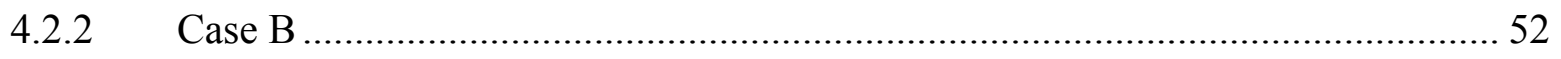

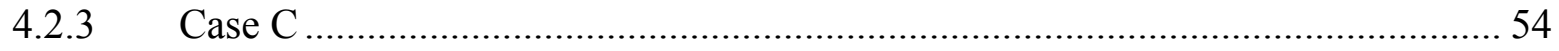

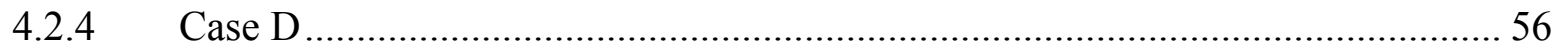

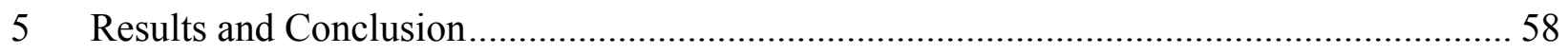

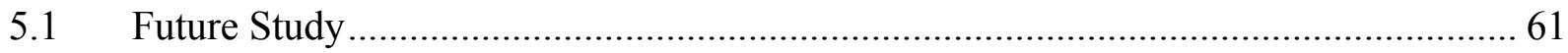

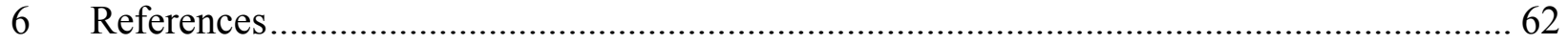

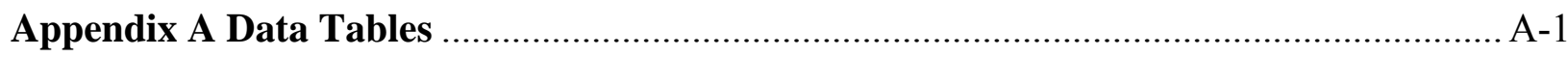

Appendix B VBA Code for the NN-CI Program............................................................ B-1

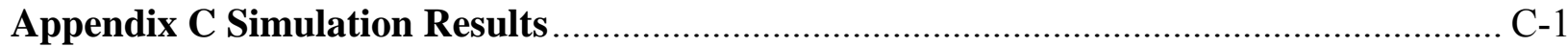

Appendix D Gradient or First Order Derivatives Calculation Code ............................ D-1

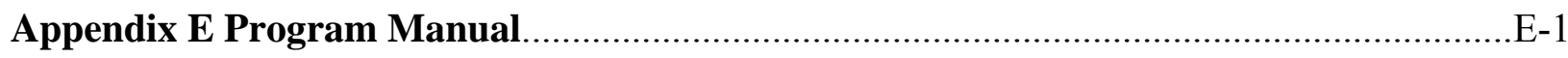

Appendix F T-test for the Means of NLR and Bootstrap.......................................F-1 


\section{List of Tables}

Table 1.1 The Truth Table for Exclusive-OR (XOR) ........................................................ 3

Table 2.1 Actual Coverage Probabilities for Nominal 95\% Confidence Intervals- L. H. Ungar et

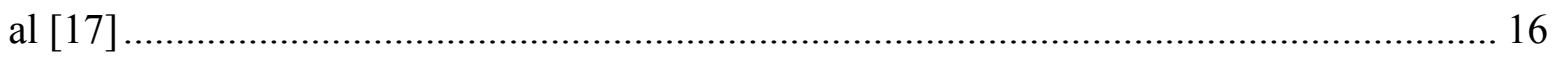

Table 2.2 Bootstrap Resampling Example [46] ............................................................. 23

Table 3.1 Parameters for Neural Network to Test Nguyen-Widrow Initialization..................... 32

Table 3.2 Mean Squared Error (MSE) with and without Nguyen-Widrow Initialization ........... 33

Table 3.3 Regression Analysis and ANOVA for Tree Data [46] ......................................... 36

Table 3.4 Median Standard Error and Standard Deviation for 25 Simulations......................... 41

Table 3.5 Neural Network and Confidence Interval Parameters for 95\% Nominal Probability . 44

Table 3.6 Results Obtained by Neural Network and Confidence Estimation Techniques with 95\%

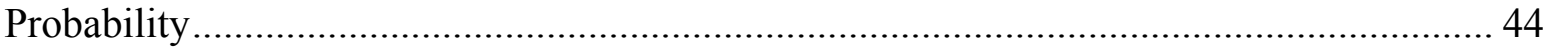

Table 3.7 Results Obtained by Neural Network and Confidence Estimation Techniques with 90\%

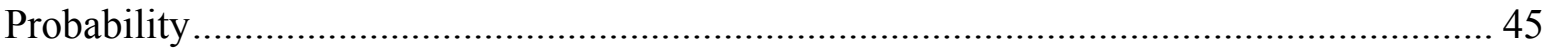

Table 4.1 Neural Network and Confidence Interval Parameters for Case A........................... 50

Table 4.2 Results for Case A: Average Taken Over 15 Simulations for 95\% Confidence Level51

Table 4.3 Results for Case A: Average Taken Over 15 Simulations for 90\% Confidence Level51

Table 4.4 Neural Network and Confidence Interval Parameters for Case B ........................... 52

Table 4.5 Results for Case B: Average Taken Over 15 Samples for 95\% Confidence Level..... 52

Table 4.6 Results for Case B: Average Taken Over 15 Samples for 90\% Confidence Level..... 53

Table 4.7 Neural Network and Confidence Interval Parameters for Case C ........................... 54

Table 4.8 Results for Case C: Average Taken Over 15 Samples for 95\% Confidence Level..... 55

Table 4.9 Results for Case C: Average Taken Over 15 Samples for 90\% Confidence Level...... 55

Table 4.10 Neural Network and Confidence Interval Parameters for Case D ......................... 57

Table 4.11 Results for Case D: Average Taken Over 15 Samples for 95\% Confidence Level.... 57

Table 4.12 Results for Case D: Average Taken Over 15 Samples for 90\% Confidence Level.... 57

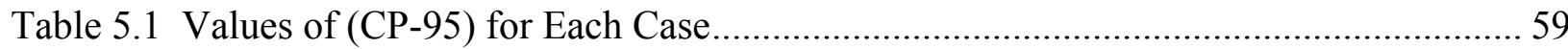

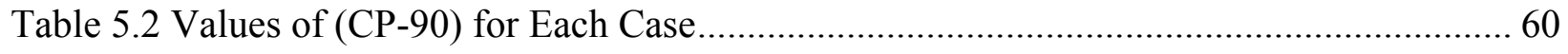

Table A-1 The Data Used for Developing Neural Network in Section 3.2.1 …..................... A-1

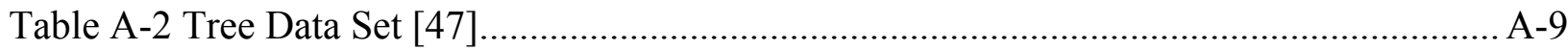

Table C-1 Simulation Results for Case A with Nominal Probability of 95\% …................... C-1 
Table C-2 Simulation Results for Case A with Nominal Probability of 90\% ........................ C-2

Table C-3 Simulation Results for Case B with Nominal Probability of 95\% ....................... C-3

Table C-4 Simulation Results for Case B with Nominal Probability of 90\% ........................ C-4

Table C-5 Simulation Results for Case C with Nominal Probability of 95\% ........................ C-5

Table C-6 Simulation Results for Case C with Nominal Probability of 90\% …..................... C-6

Table C-7 Simulation Results for Case D with Nominal Probability of 95\% ....................... C-7

Table C-8 Simulation Results for Case D with Nominal Probability of 90\% ........................ C-8

Table E-1 Inputs and the Possible Values to the Program...................................................E-2

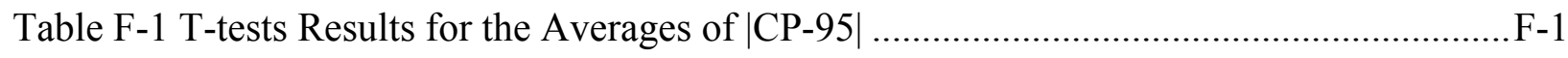

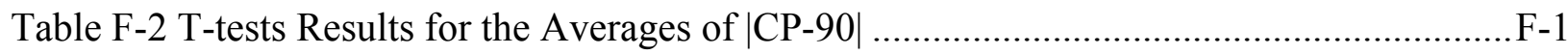




\section{List of Figures}

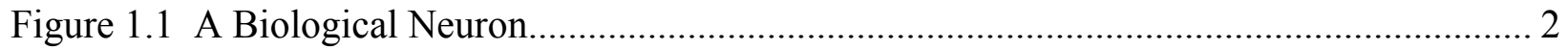

Figure 1.2 Feed-forward Network (3-2-1) Architecture ......................................................... 3

Figure 1.3 A Feed-forward Network with One Hidden Layer [5] ............................................. 5

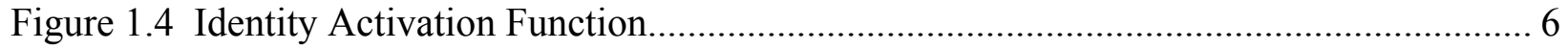

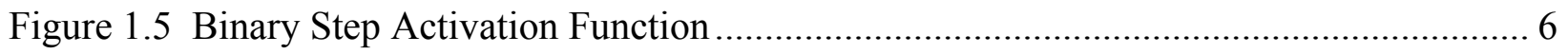

Figure 1.6 Logistic Activation Function ................................................................................ 7

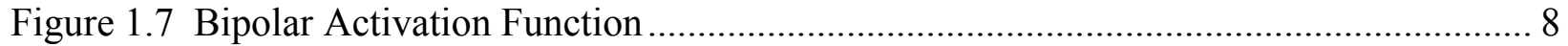

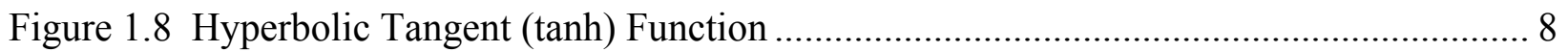

Figure 1.9 Steps of the Backpropagation Algorithm [5]......................................................... 11

Figure 3.1 Flowchart of the Backpropagation Algorithm...................................................... 28

Figure 3.2 MSE for Training and Test Data, and R-square for Training and Test Data Using 20,

30, and 40 Epochs .................................................................................................... 30

Figure 3.3 MSE and Average MSE for Neural Networks with and without NWI ...................... 33

Figure 3.4 Calculations of Confidence Intervals for Linear Regression in a Spreadsheet ........... 36

Figure 3.5 Trunk Girth vs. Weight for the Tree Data [46] using Regression Predicted Weight

Values Given By Dots, Actual Weight Values Given By Stars, and Solid Lines 95\%

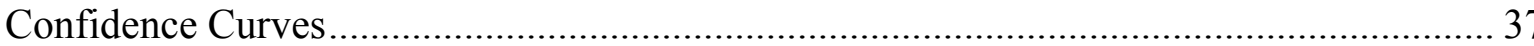

Figure 3.6 Trunk Girth vs. Weight Using Neural Networks with Confidence Curves via Non-

Linear Regression-with and without the Correction Factor (CF) with Confidence Level 95\%

Figure 3.7 Trunk Girth vs. Weight Using Neural Networks with Confidence Curves Using

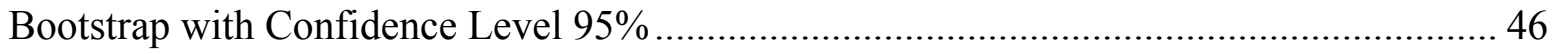

Figure 3.8 Trunk Girth vs. Weight Using Neural Networks with Confidence Curves Using MLE

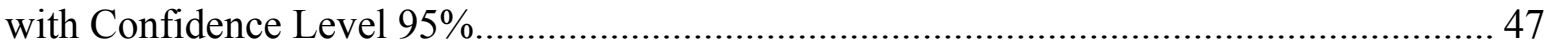

Figure 3.9 Confidence Curves Obtained via All Techniques .................................................... 48

Figure 4.1 Confidence Intervals by all the Techniques for Case B ............................................. 53

Figure 4.2 Confidence Curves Obtained by all Techniques for Case C at 95\% Confidence Level

Figure 5.1 CP - 95 Results for Each Technique for Respective Data Sets Shown in this Order:

NLR, Bootstrapping, and MLE 59 
Figure 5.2 CP - 90 Results for Each Technique for Respective Data Sets Shown in this Order:

NLR, Bootstrapping and MLE. 60

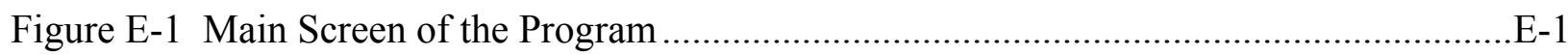

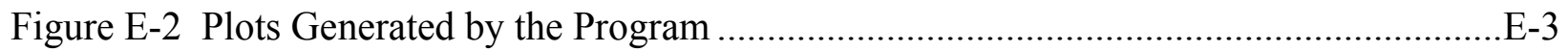

Figure E-3 Confidence Intervals Parameters Form …………………………………….........

Figure F-1 T-test for Comparing the Means ........................................................................... 


\section{Table of Nomenclature}

Notations: Also refer Figure 1.3

$n \quad$ Number of input units.

$n o b \quad$ Number of obeservations or training pairs.

$n h \quad$ Number of hidden layer neurons.

noy Number of output layer neurons.

$x_{i} \quad$ Activations of units $\mathrm{X}_{\mathrm{i}}$ :

For input units $\mathrm{X}_{\mathrm{i}}$, $x_{i}=$ input signal;

$t_{k} \quad$ Target values.

$w_{i j} \quad$ Weights between the input layer and the hidden layer;

$w_{0 j} \quad$ Bias term applied to the hidden layer.

$v_{j k} \quad$ Weights between the hidden layer and the output layer;

$v_{0 k} \quad$ Bias term applied to the output layer.

Note: Bias terms are weights to the connections whose ouput is always 1 .

$\alpha \quad$ Learning rate.

$\delta_{j}, \delta_{k} \quad$ Part of error correction weight adjustment 


\section{Introduction}

\subsection{Neural Networks}

The simple application of Artificial Neural Networks (ANNs) has made them one of the most widespread techniques to predict, forecast, or classify data. Artificial neural networks are also known as neural networks. ANN's capability to detect a pattern or relationship in the given input data is much faster and less labor intensive than conventional methods. Therefore, ANNs are being used as an alternative to traditional models for a range of applications, such as, medical $[1,35]$, cost estimation [2], ecology [3], coastal research [36], forecasting [41].

\subsubsection{What is an Artificial Neural Network?}

An artificial neural network (ANN) is an information-processing model that is influenced and motivated by the way the brain works. An ANN is an attempt to represent structure of the brain, which is composed of a large number of highly interconnected processing elements or neurons. Scientists have learned that a brain learns by adjusting the synaptic connections between the neurons. There is a visible similarity between people and ANN, for example, people learn by example and so does ANN. ANNs also adjust the connections between the neurons for learning. If trained with preprocessed data and a proper structure, ANNs may display the capability of generalizing the data and producing good results for unseen data.

There is no universally accepted definition of an ANN, but Online Encyclopædia Britannica $C$ [4] defines it as:

"Computer architecture in which a number of processors are interconnected in a manner suggestive of the connections between neurons in a human brain and which is able to learn by a process of trial and error -- called also neural network."

\subsubsection{Biological Neural Network}

A biological neuron has three components that are important in understanding an artificial neural net: dendrites, soma, and axon as mentioned by Laurene Fausett [5]. The input signals are electrical impulses that are transmitted across a synaptic gap. The many dendrites present receive signals from neurons. This incoming information is summed up by soma and then delivered along the neuron's axon to the dendrites at its end. The information will again be passed if the stimulation caused by the signals has exceeded a certain threshold. If a simulation 
fails to cross the threshold, it would not be passed further. A biological neuron can be represented as shown in Figure 1.1.

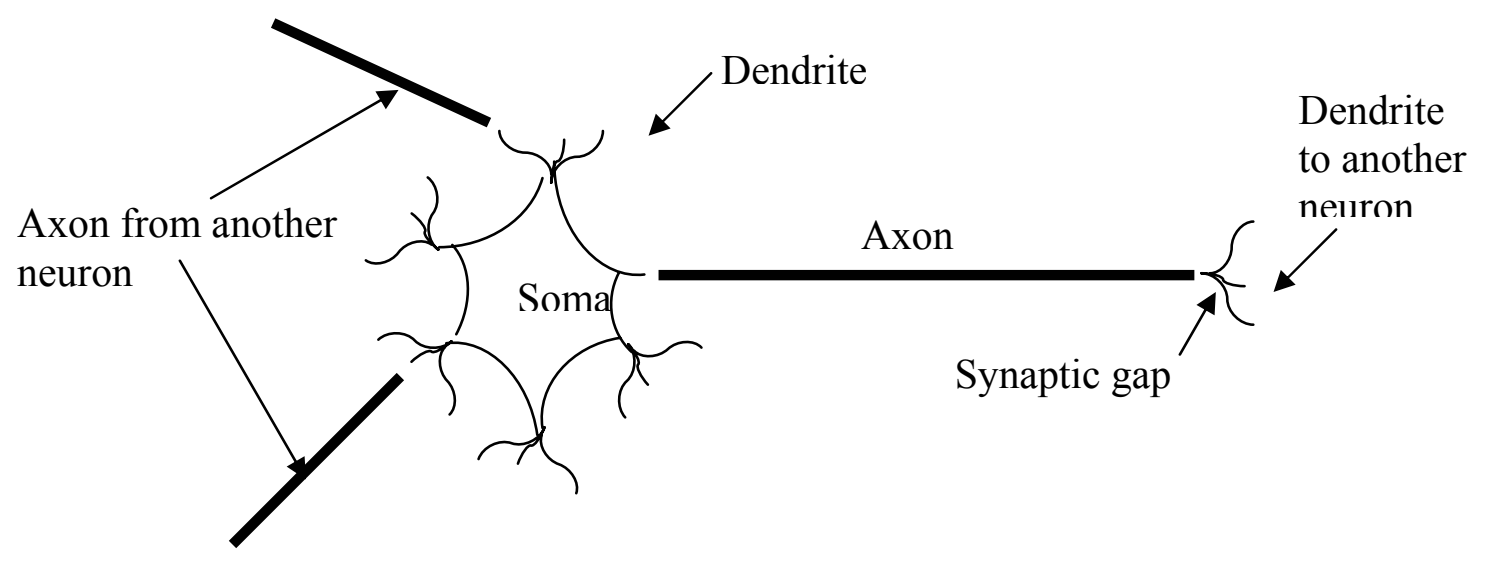

Figure 1.1 A Biological Neuron

\subsubsection{Historical Background}

With the arrival of modern electronics and computers, attempts to replicate the thinking process was inevitable. Warren McCulloch and Walter Pitts were first to recognize that combining simple neurons into neural system might increase computational power [6, 7]. McCulloch and Pitts developed a neural network for an electric circuit in 1943. Later in their research they dealt with translation and rotation invariant pattern recognition.

In 1949, Donald Hebb designed the first learning law for ANNs and wrote the book Organization of Behavior [7]. He presented a theory that the strength of the connection between two neurons should be increased every time they were used or active. Around 1958, John von Newumann, the "father of modern computing," suggested using telegraph relays or vacuum tubes to emulate simple neuron functions. Soon after that Frank Rosenblatt started working on a class of ANNs called perceptrons. The perceptron was found to be useful in classifying inputs into one class.It is the oldest neural network still in use.

In 1969, Minsky and Papert, published a book titled Perceptrons, in which they proved that perceptron was limited and unable to solve the XOR (exclusive-or) problem. The XOR is not linearly separable, while OR and AND are linearly separable. The truth table for XOR is given in Table 1.1. In 1982, John Hopfield developed a number of neural networks based on fixed weights, which brought popular attention to neural nets. Also, optical neural nets by Farhat, Psaltis et al. [1985], and VLSI implementations by Sivilatti, Mahowald, and Mead [1987] were 
developed in the 1990s. Faster computational abilities and superior hardware are the reasons for renewed interest in ANN, and today, discussions on ANNs are commonplace.

Table 1.1 The Truth Table for Exclusive-OR (XOR)

\begin{tabular}{|c|c|c|}
\hline $\mathbf{a}$ & $\mathbf{b}$ & $\mathbf{a}$ XOR b \\
\hline 0 & 0 & 0 \\
\hline 0 & 1 & 1 \\
\hline 1 & 0 & 1 \\
\hline 1 & 1 & 0 \\
\hline
\end{tabular}

\subsubsection{Architecture of a Feed-forward Network}

A feed-forward ANN is a straightforward network that sends signals from one layer to another layer, which means the output of one layer does not affect the same layer. The first layer receives the input, and each neuron in a layer is fully connected to all other neurons in the following layer. The output of the last layer is the output of the network. All layers between the input and output layers are called hidden layers. It is a common practice to use either one or two hidden layers, and a single hidden layer was selected as the problems investigated were not complex. A feed forward network with a configuration as one input layer with three inputs, one hidden layer with two nodes, and one output layer with a single output (3-2-1) can be represented as shown in Figure 1.2.

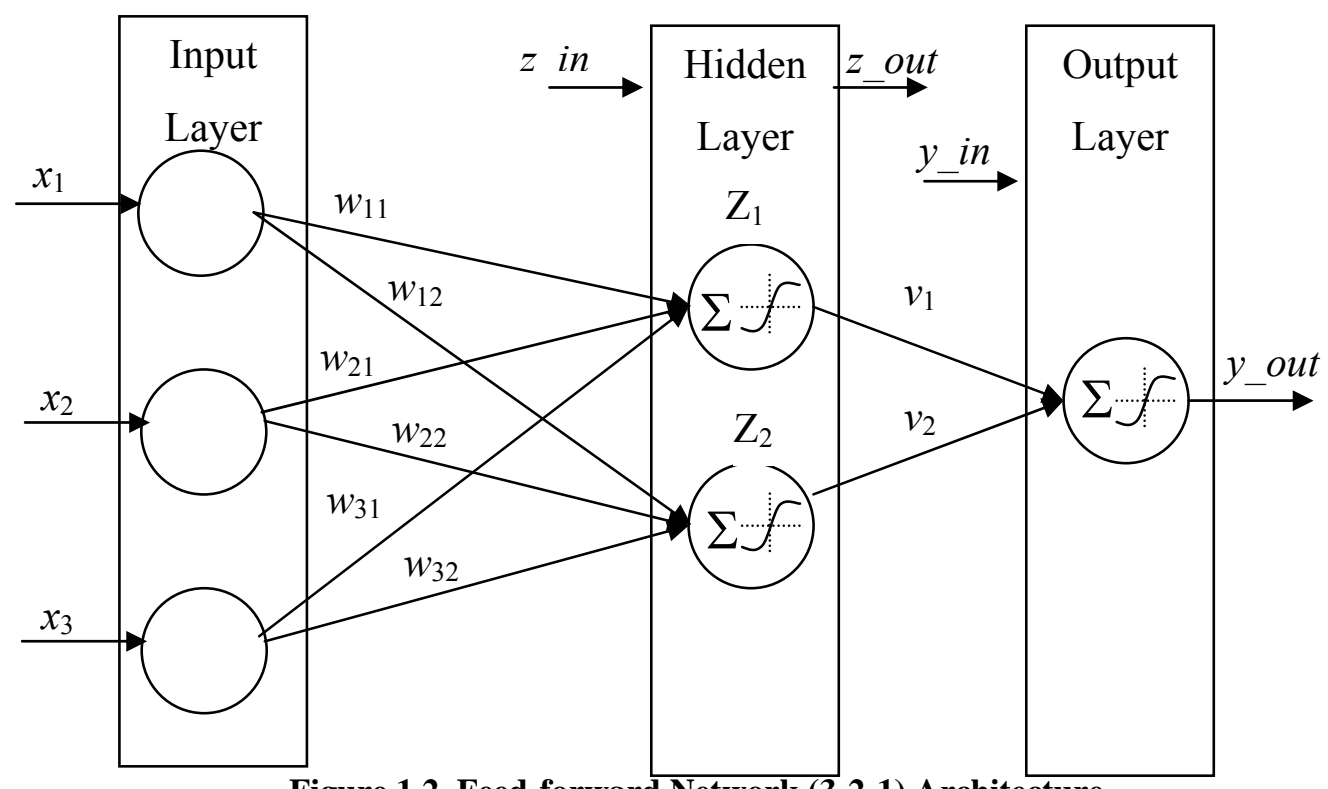

Figure 1.2 Feed-forward Network (3-2-1) Architecture 
Figure 1.2 represents a three-two-one feed-forward network structure with three input neurons, two hidden layer neurons, and one output layer neuron. The input layer receives signals as $\mathrm{X}_{1}, \mathrm{X}_{2}$, and $\mathrm{X}_{3}$. Random or fixed weights are assigned to the connections between all the neurons in all the layers. The weight matrices are shown in Equation (1). Matrix $W$ denotes the weights between the input layer and the hidden layer, and matrix $V$ denotes the weights between the hidden layer and the output layer.

$$
W=\left[\begin{array}{ll}
w_{11} & w_{12} \\
w_{21} & w_{22} \\
w_{31} & w_{32}
\end{array}\right] ; \quad V=\left[\begin{array}{l}
v_{1} \\
v_{2}
\end{array}\right]
$$

Matrix $W$ is multiplied with the input signals and then summed up in the hidden layer. An activation function, given in Equation (2), is applied to this summation to give new input signals for the next layer:

$$
y=f(x)
$$

where,

$\mathrm{y}=$ output of the function,

$f()=$ linear identity or non-linear function, and

$\mathrm{x}=$ input to the function.

This new information is again fed-forward and multiplied by the weights between hidden and output layer, i.e., matrix $V$. This multiplied signal is again sent through the activation function to give the output or result of the network. A feed-forward network for one hidden layer is depicted in Figure 1.3. 
$n \quad$ Number of input units

$n h \quad$ Number of hidden layer neurons

$x_{\mathrm{i}} \quad$ Activation of units $\mathrm{X}_{\mathrm{i}}$

For input units $X_{i}$

$x_{i}=$ input signal

$w_{i j} \quad$ Weights between the input layer and the hidden layer

$v_{j k} \quad$ Weights between the hidden layer and the output layer

$z_{-}$in $_{j}$ Input to the hidden layer:

$$
z_{-} i n_{j}=\sum_{i=1}^{n} w_{i j} \cdot x_{i}
$$

$z_{-}$out ${ }_{j}$ Output of the hidden neurons:

$$
z_{-} \text {out }_{j}=f\left(z_{-} \text {in }{ }_{j}\right)
$$

$y_{-} i n_{k}$ Input to the output layer:

$$
\mathrm{y} \_ \text {in }_{\mathrm{k}}=\sum_{j=1}^{n h} v_{j k} \cdot z_{-} \text {out }{ }_{j}
$$

Note: If there is only one output unit, then subscript $\mathrm{k}$ is removed $y_{-}$out $_{k}$ Output of the network:

$$
y_{-} \text {out }_{k}=f\left(y_{-} i n_{k}\right)
$$

Figure 1.3 A Feed-forward Network with One Hidden Layer [5]

\subsubsection{Activation Function}

Activation functions are needed to introduce nonlinearity into the network. Without nonlinearity, hidden units would not be and more powerful than just plain perceptrons (which do not have any hidden units, just input and output units).A linear function of linear functions is always a linear function. However, it is the capability to represent nonlinear functions that makes multilayer networks so powerful. For backpropagation learning, the activation function must be differentiable, and it is helpful if the function is bounded.

\section{(i) Identity Activation Function}

For the input layer identity, activation function is used as given in Equation (3) and shown in Figure 1.4. It is a common practice to use the same activation function for all the neurons in a layer. An identity activation function is typically used for the output and input layer.

$$
f(x)=x \text { for all } x
$$




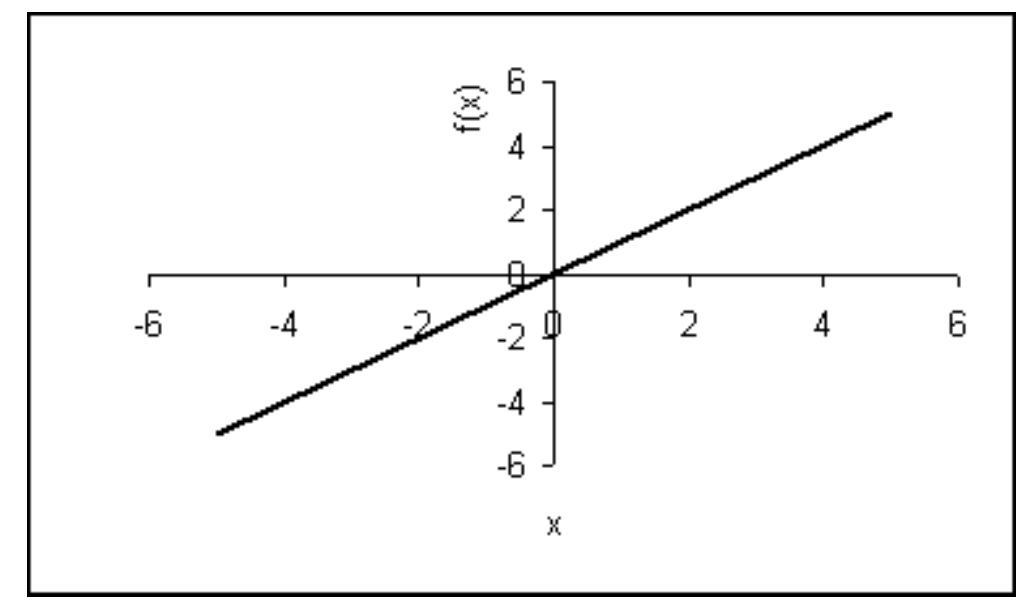

Figure 1.4 Identity Activation Function

\section{(ii) Binary Step Function}

The binary step function is also known as threshold function. The output of this function is either bipolar ( 1 or -1$)$ or binary ( 1 or 0$)$. This function applies a simple rule, that is, if the input exceeds its threshold, then the output is positive, otherwise it is negative. The binary step function is given in Equation (4) and depicted in Figure 1.5.

$$
f(x)= \begin{cases}1 & \text { if } x \geq \theta ; \\ 0 & \text { if } x<\theta .\end{cases}
$$

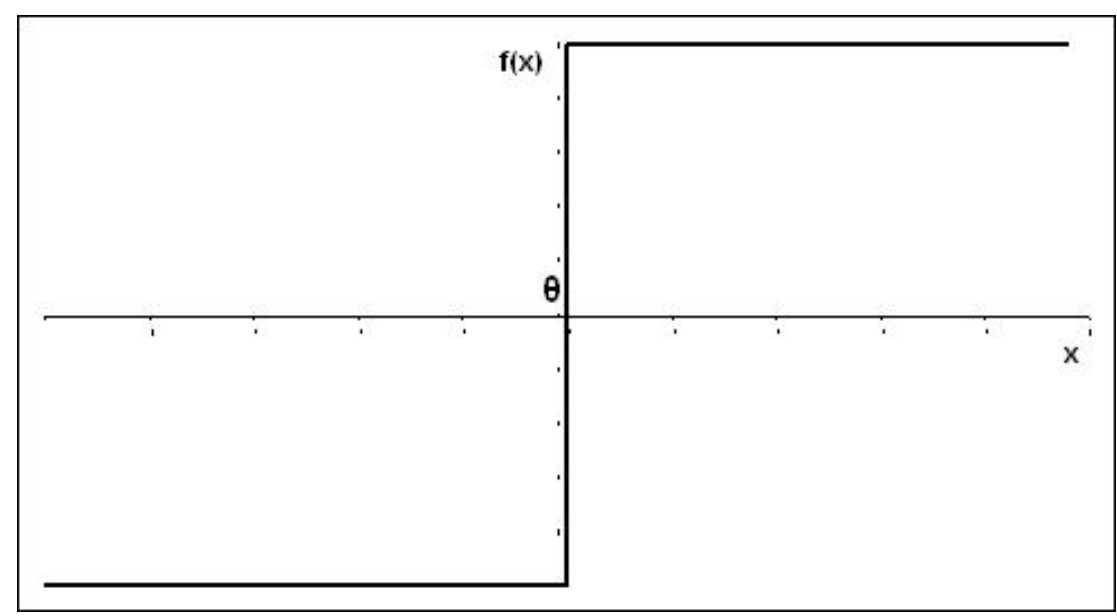

Figure 1.5 Binary Step Activation Function 


\section{(iii) Binary Sigmoid Function}

This function is also called logistic function with an output range from zero to one. This is the most common activation function used and this function was used for the hidden layer. It is in the form given in Equation (5) and is illustrated in Figure 1.6.

$$
f(x)=\frac{1}{1+e^{(-x)}}
$$

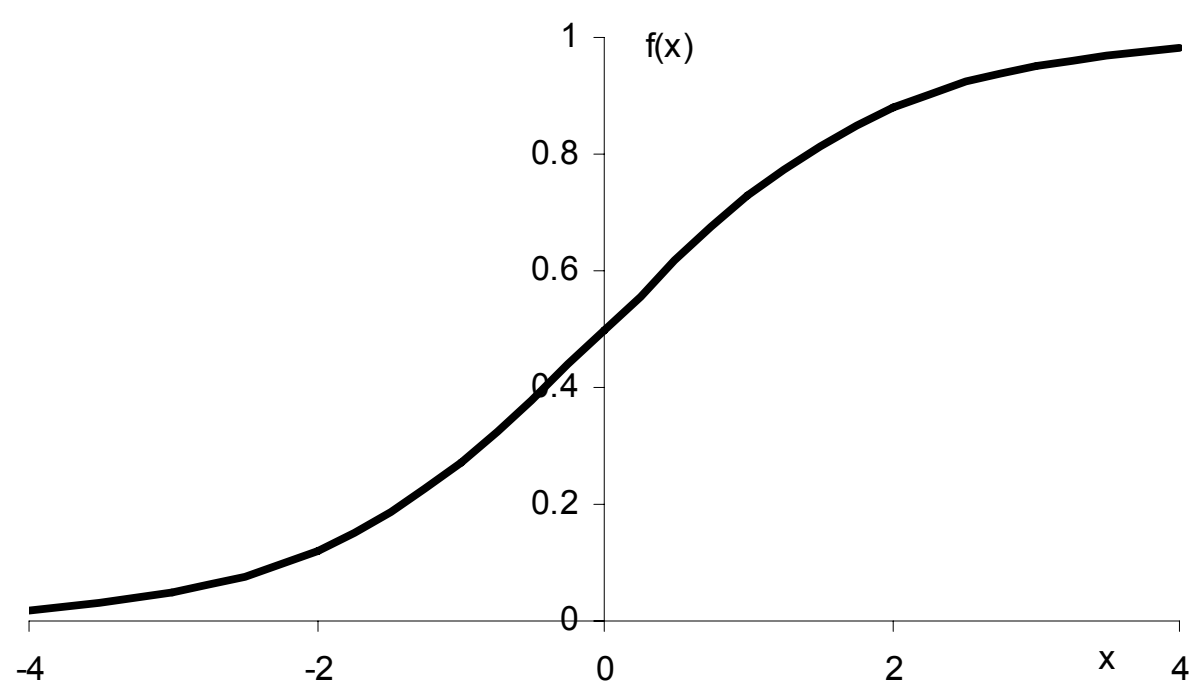

Figure 1.6 Logistic Activation Function

(iv) Bipolar Sigmoid Function

The most common form of bipolar sigmoid function is given in Equation (6) and is depicted in Figure 1.7. The range commonly used is -1 to 1 and therefore it is called a bipolar sigmoid function.

$$
f(x)=\frac{1-e^{-x}}{1+e^{-x}}
$$




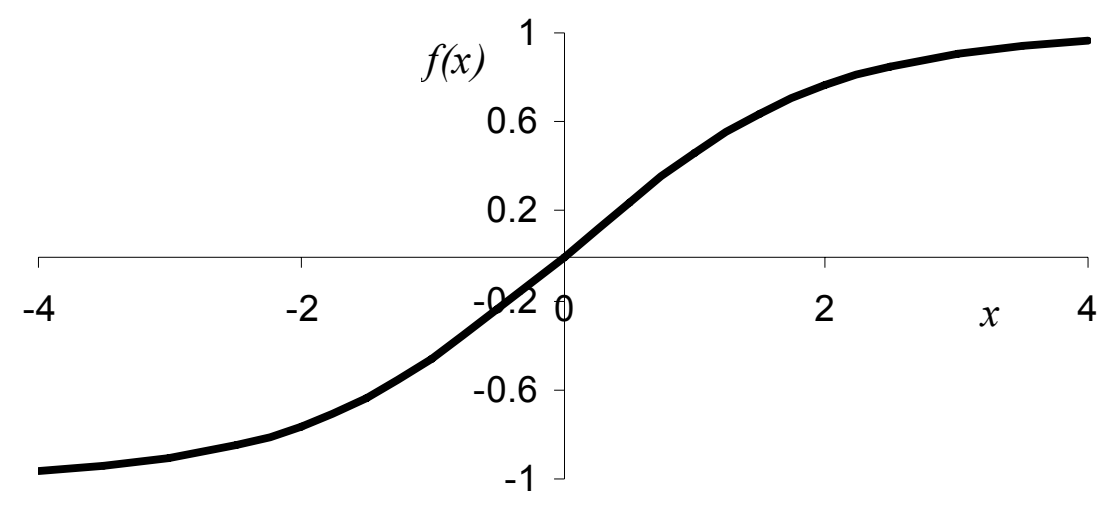

Figure 1.7 Bipolar Activation Function

(v) Hyperbolic Tangent Function

TanH has a similar sigmoid shape to the logistic function, but values are spread through the interval $[-1,1]$, rather than $[0,1]$. This function is given by Equation (7), and it takes the shape shown in Figure 1.8.

$$
f(x)=\frac{e^{x}-e^{-x}}{e^{x}+e^{-x}}
$$

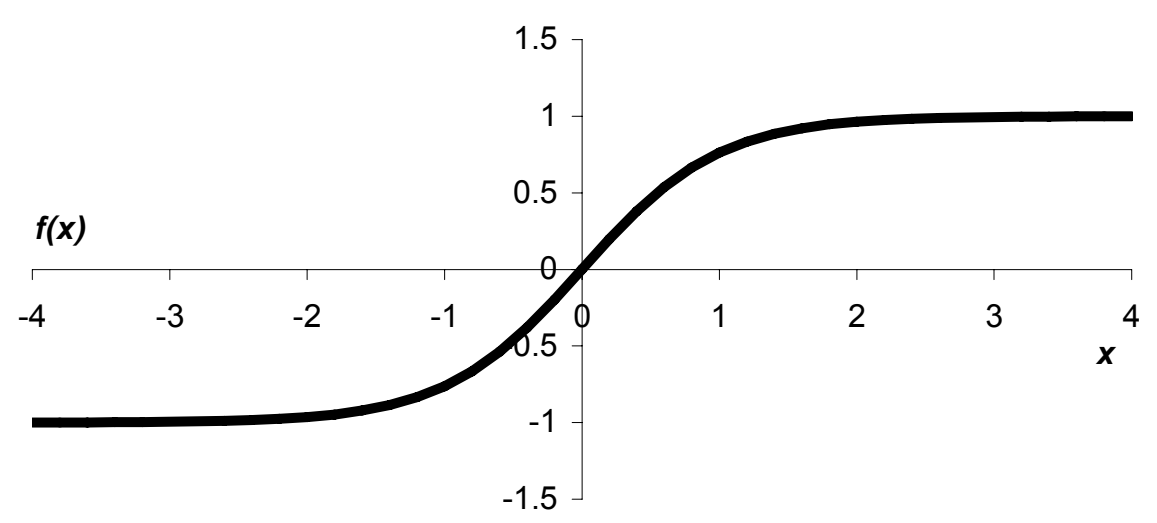

Figure 1.8 Hyperbolic Tangent (tanh) Function

The most common of activation function used in the construction of ANNs for the hidden layer is the binary sigmoid function and tanh function. With sigmoid units, a small change in the weights will usually produce a change in the outputs, which makes it possible to tell whether that change in the weights is good or bad. Also, the sigmoid function is global in the sense that it 
divides the feature space into two halves, one where the response is approaching one and another where it is approaching zero. For the output layer, usually a linear function is used [5].

\subsubsection{Network Learning}

The brain updates itself whenever it receives a new pattern. This updating helps the brain to solve a problem related to that pattern. ANNs are developed to make an attempt to imitate the brain which is the reason ANNs are sometimes called machine-learning algorithms [8]. However, in the case of ANNs, updating occurs only for the weights used in the network and not the whole network. An ANN learns new patterns by adjusting these weights connected between all the layers.

The learning procedure, also known as a training algorithm, and the architecture of an ANN determine its learning ability. The architecture and the training algorithm may vary from requirement to requirement but mostly they falls in two major categories - supervised (reinforcement learning) and unsupervised learning.

\section{(i) Supervised (Reinforcement Learning)}

In supervised learning, the learning rule is provided with the training set consisting of an input and output or target sample set as given in Equation (8). The inputs are applied to the network to calculate the network outputs, which are then compared to the targets. After completion of that step, the learning rule is used to adjust the weights of the network to decrease the error between the network outputs and the targets:

$$
\left\{X_{1}, T_{1}\right\},\left\{X_{2}, T_{2}\right\},\left\{X_{3}, T_{3}\right\}, \ldots \ldots,\left\{X_{n o b}, T_{n o b}\right\}
$$

where,

$X$ = input matrix,

$T=$ target or output matrix, and

$n o b=$ number of observation or samples.

\section{(ii) Unsupervised Learning}

In this approach, no sample targets or outputs are provided to the network against which the network can measure its performance for given inputs. The network modifies the weights in response to network inputs only. As is mentioned in the Matlab neural network 
toolbox online documentation [9], the network categorizes the input patterns into a finite number of classes.

As stated in the online documentation on neural networks by West Virginia University [10], the Hopfield net, the Hamming net, the Bidirectional Associative Memory (BAM), the Delta rule, and the backpropagation algorithm are considered to be supervised learning networks. The Adaptive Resonance Theory (ART) by Carpenter and Grossberg and Kohonen's selforganizing maps are unsupervised learning networks [10].

The backpropagation algorithm is the most known and widely used learning procedure. Two reasons for its popularity in the neural network fraternity are the robustness and stability of the network. There is a vast literature available on the backpropagation algorithm and the backpropagation algorithm was used as the learning procedure.

\subsubsection{Backpropagation Algorithm}

The limitation to solve a wide variety of problems using single-layer neural networks was an important factor in the decrease of interests in neural networks in the 1970s. The discovery of the backpropagation or the generalized delta rule training methods for multi-layer networks by D. E. Rumelhart, G. E. Hinton, and R. J. Williams [11] helped rejuvenate neural network interest.

The term backpropagation refers to the manner in which a backward pass of error to each internal neuron within the network is used to calculate weight gradients for that neuron. The learning of the network is accomplished by alternately propagating forward the activations and propagating backward the instantaneous errors. The network weights are moved along the negative of the gradient of the performance function.

The backpropagation algorithm is shown in Figure 1.9 and it consists of these important steps:

(i) initializing of the weights,

(ii) executing the feed-forward network,

(iii) back propagating of the error and adjustment of the weights, and

(iv) repeating of these steps until the difference between the target and the network output values is within an acceptable range. 
Algorithm:

Step 0. Initialize weights to small random values.

Step 1 . Repeat steps 2-8 until the stopping condition is false.

Step 2. Repeat steps 3-7 for $m=1$ to nob:

Step 3. Each hidden neuron $\left(Z_{j}, j=1, \ldots ., n h\right)$ sums its weighted input signals:

$$
z_{-} i n_{j}=w_{o j}+\sum_{i=1}^{n} w_{i j} . x_{i}
$$

Output signal of the hidden neurons:

$$
z_{-} \text {out } t_{j}=f\left(z_{-} i n_{j}\right) \text {. }
$$

Step 4. Each output neuron $\left(Y_{k}, k=1, \ldots . ., n o y\right)$ sums its weighted input signals:

$$
y_{-} i n_{k}=v_{o k}+\sum_{j=1}^{n h} v_{j k} z \text { _out } j \text {. }
$$

Note: If there is only one output unit then subscript $k$ is removed

Output of the network:

$$
y_{-} \text {out } k=f\left(y_{-} \text {in }_{k}\right) \text {. }
$$

Step 5. Each output layer neuron computes its error information: $\delta k=\left(t k-y_{-}\right.$outk $) \cdot f^{\prime}\left(y_{-}\right.$ink $)$, then it calculates its weight correction term: $\Delta v j k=\alpha . \delta k . z \_o u t j, \quad$ then it calculates its bias correction term: $\Delta v 0 k=\alpha . \delta k$, and then $\delta k$ is sent back to the hidden layer.

Step 6. Each hidden neuron sums its delta inputs:

$\delta_{-} i n_{j}=\sum_{k=1}^{n o y} \delta_{k} \cdot v_{j k}$, then it calculates its error information term:

$\delta_{j}=\delta \_i n_{j} . f^{\prime}\left(z_{-} i n_{j}\right)$, then it calculates its weight correction term: $\Delta w_{i j}=\alpha . \delta_{j . x_{i}}$, and then it calculates its bias correction term: $\Delta w_{0 j}=\alpha . \delta_{j}$.

Step 7. Each hidden layer neuron updates its bias and weights:

$w_{\mathrm{ij}}($ new $)=w_{\mathrm{ij}}($ old $)+\Delta w_{\mathrm{ij}}$

Each output layer neuron updates its bias and weights:

$v_{\mathrm{jk}}($ new $)=v_{\mathrm{jk}}($ old $)+\Delta v_{\mathrm{jk}}$

Step 8. Check the stopping condition.

Figure 1.9 Steps of the Backpropagation Algorithm [5] 
The derivation of the learning rule is adopted from Laurene Fausett's book, Fundamentals of Neural Networks [7] given as follows. The error to be minimized is $E=0.5 \sum_{k}\left[t_{k}-y_{-} \text {out } t_{k}\right]^{2}$. By using the chain rule, we have

$$
\begin{aligned}
\frac{\partial E}{\partial V_{j k}} & =\frac{\partial}{\partial V_{j k}} 0.5 \sum_{k}\left[t_{k}-y_{-} \text {out } t_{k}\right]^{2}=\frac{\partial}{\partial V_{j k}} 0.5 \sum_{k}\left[t_{k}-f\left(y_{-} \text {in } n_{k}\right)\right]^{2} \\
& =-\left[t_{k}-y_{-} \text {out } t_{k}\right] \frac{\partial}{\partial V_{j k}} f\left(y_{-} \text {in } n_{k}\right)=-\left[t_{k}-y_{-} \text {out } t_{k}\right] f^{\prime}\left(y_{-} \text {in } k\right) \frac{\partial}{\partial V_{j k}} f\left(y_{-} \text {ink }\right) \\
& =-\left[t_{k}-y_{-} \text {out } t_{k}\right] f^{\prime}\left(y_{-} \text {ink }\right) z_{-} \text {out } t_{j}
\end{aligned}
$$

Define: $\delta_{k}=\left[t_{k}-y_{-} o u t_{k}\right] f^{\prime}\left(y_{-}{ }_{i n k}\right)$

$$
\begin{aligned}
& \frac{\partial E}{\partial W_{i j}}=-\sum_{k}\left[t_{k}-y_{-} \text {out } t_{k} \frac{\partial}{\partial w_{i j}} y_{-} \text {outk }=-\sum_{k}\left[t_{k}-y_{-} \text {out } t_{k} f^{\prime}\left(y_{-} \text {ink }\right) \frac{\partial}{\partial w_{i j}} y_{-}{ }^{i n k}\right.\right. \\
& =-\sum_{k} \delta_{k} V_{j k} \frac{\partial}{\partial w_{i j}} z_{-} \text {out } t_{j} \quad=-\sum_{k} \delta_{k} V_{j k} f^{\prime}\left(z_{-} i n_{j}\right)\left[x_{i}\right]
\end{aligned}
$$

Define: $\delta_{j}=\sum_{k} \delta_{k} V_{j k} f^{\prime}\left(z_{-} i n_{j}\right)$

Using this chain rule the gradients or partial derivatives of error with respect to the weights were found. With this gradient the weight correction terms were found and the old weights were updated. This process of finding error information terms and updating of weights is called as backpropagation.

\subsection{Need of Confidence Intervals for Neural Networks}

It is a common perception that ANN is a "black box" that is able to predict an output pattern when it recognizes the input pattern. The desired output or result is entirely based on the behavior of the neither the user is never sure of the results. Nevertheless, ANNs are used widely because of their capability of picking random or sudden changes in the pattern. Results of the networks suffer, however from uncertainty due to improper preparation of data and design of a neural network. Unfortunately, ANN models do not provide assurance, accuracy, or reliability of their output.

D. Lowe and K. Zapart [28] stated that the application of neural network approaches to the real world problems has now approached to a situation where they are being considered for the functionality 0 fkey embedded components in safety-critical and mission-critical systems. 
They also noted that in critical situations the ability of ANNs to classify, forecast, or regress was not adequate. The ANNs must quantify the minimum additional knowledge to produce confidence intervals, which are necessary to quantify the accuracy or reliability of the neural network performance.

The ability to estimate the uncertainty of predictions is important for many reasons. For example, in process control, when designing controllers and verifying their performance, it is useful to know the accuracy of the model used by the controller. Model accuracy is an important factor for model selection. J. Schumann et. al. [27] mention testing the practical feasibility of a neural network-based controller for aircraft at NASA, for manned F-15 active aircraft based at Dryden Flight Research Center, as well as for a C-17 transport aircraft. On-line trained neural networks within the flight controller can adapt to sudden damage of a control surface to prevent fatal accidents.

J. Schumann, P. Gupta, and S. Nelson [27] states that the main criticism against inclusion of on-line trained neural networks in safety-critical applications has been their non-determinism. Therefore, it is very important that the predictions of the neural networks provide some information on the reliability and the confidence intervals on the output of the neural networks and can provide a precision check.

S. L. Ho et. al. [41] used ANNs for time-series forecasting of the solder paste deposition process, and they constructed confidence intervals on the output of the neural networks using non-linear regression. They noted that process control using neural networks with confidence bounds provided more quality information for better decision making and continuous improvement.

\subsubsection{Confidence Interval}

A confidence interval is a range of values that has a specified probability of containing the parameter being estimated from a given set of sample data as given in V. J. Easton et. al. [28]. The width of the confidence interval gives us some idea of how uncertain we are about the estimated or unknown parameter. A wide interval may indicate that more data should be collected before anything very definite can be said about the parameter.

The most widely used confidence intervals are the $95 \%$ and $99 \%$ confidence intervals, which have 0.95 and 0.99 probabilities of containing the parameter respectively. If the user 
selects a $95 \%$ confidence level, which can also be stated as $(1-\alpha)$ where $\alpha$ is acceptable risk of being wrong and equals to 0.05 , confidence level then it would mean that if the same population is sampled on numerous occasions and confidence interval estimates are made on each occasion, the resulting intervals would bracket the true population parameter in approximately $95 \%$ of the cases. A confidence interval has the specified probability of containing the parameter if the sample data on which it is based is the only information available about the value of the parameter.

\subsection{Research Method}

This research was carried out as given in the following steps:

(i) Literature search: There are four main techniques available to compute the confidence intervals: non-linear regression (NLR), bootstrapping (boot), maximum likelihood estimation (MLE), and Bayesian estimation. Only NLR, bootstrap, and MLE were coded in a programming language and analyzed, as Bayesian estimation was too complex to be coded.

(ii) Develop a neural network model: A basic multi-layer perceptron feed-forward network with the backpropagation algorithm for weight adjustment was developed. A model was developed that would train and test the given data. The output neuron or the output unit and the number of hidden layers were limited to one.

(iii) Coding confidence interval models: Three different models, NLR, bootstrap, and MLE, were coded in Visual Basic programming language to provide good user interface.

(iv) Detailed analysis: These models were tested and analyzed on different data sets. Additional noise was added to test the models for errors other than the errors in the original data.

(v) Conclusion: Results on the different options for calculating confidence intervals on neural networks with reliability were presented. 


\section{Literature Review}

\subsection{Introduction}

A literature search was done to analyze the existing techniques for computing the confidence intervals of the output of neural networks. Fundamentals of Neural Networks by Laurene Fausett $[5,7]$ was studied to learn more about neural networks and their applications. Computer Systems That Learn by S. M. Weiss and C. A. Kulikowski [12] gave a mathematical view on neural networks.Papers were collected from the IEEE Transactions on Neural Networks, ScienceDirect® by Elsevier, and various authors’ personal WebPages.

The techniques available to compute confidence intervals on neural networks were: nonlinear regression (NLR), bootstrapping, the maximum-likelihood estimation (MLE), multilinear regression (MLR), and the Bayesian inference. However, the application of the MLR technique was use only once [37], therefore, it was not included in this research. The Bayesian inference estimation technique was complex and excluded from the research. The results for the base case were compared with linear regression, non-linear regression, bootstrapping, and the maximumlikelihood is

\subsubsection{Non-linear Regression (NLR)}

G. Chryssolouris, M. Lee, and A. Ramsey [16] were among the first to derive an estimate of a neural network's accuracy as an empirical modeling tool. They defined confidence interval as : " a prediction of the range of the output of a model where the actual value exists". A brief background of calculating confidence interval for parameterized models with and without noisy data was presented. They have extended the confidence interval calculation for neural networks and also provided an example of the method. These data were not available for this research. The authors assumed that the error was normally distributed. The authors successfully observed that $85 \%$ of predictions by the neural network were within confidence intervals.

L. H. Ungar, R. D. D. Veaux, and E. Rosengarten [17] compared the ability of non-linear regression and Bayesian estimation to provide accurate confidence intervals. The authors also examined the coverage probabilities of the confidence intervals with computational costs and practical implementation. The paper focused on a feed-forward network and backpropagation algorithm with sigmoidal function as its activation function. This paper presented a comparison of non-linear regression and the Bayesian estimation techniques on the data generated using sine 
wave function with Gaussian noise with standard deviation of one, and a function as given in Equation (9). The authors concluded that the Bayesian method of model estimation should be tried on more data before it can be trusted. The results from this paper of actual coverage probabilities (CP) for nominal 95\% confidence intervals on 1000 test data points are given in Table 2.1. CP is the probability that the target (true value) of an input pattern lies within the confidence intervals. The observed mean CP should be near the "nominal" value of confidence level if the confidence estimation technique is performing well. The mean CP is a measure of global performance, i.e., the average quality of the uncertainty estimate. The CP can be calculated by determining whether the true parameter values lie within the confidence regions and confidence intervals.

$$
y=\left(10 \sin \left(\pi x_{1} x_{2}\right)+20\left(x_{3}-0.5\right)^{2}+10 x_{4}+5 x_{5}+\varepsilon\right) / 25
$$

Table 2.1 Actual Coverage Probabilities for Nominal 95\% Confidence Intervals- L. H. Ungar et al [17]

\begin{tabular}{|c|c|c|c|c|}
\hline & \multicolumn{2}{|c|}{ Non-linear Regression } & \multicolumn{2}{|c|}{ Bayesian } \\
\hline & $\mathrm{CP}$ & $|\mathrm{CP}-95|$ & $\mathrm{CP}$ & $|\mathrm{CP}-95|$ \\
\hline Sine wave & 98.8 & 3.8 & 98.4 & 3.4 \\
\hline Equation (9) & 100.0 & 5.0 & 100.0 & 5.00 \\
\hline
\end{tabular}

R. Shao, E. B. Martin, J. Zhang, and A. J. Morris [14] discuss and analyze non-linear regression as a tool to calculate confidence intervals. However, the authors are critical of the distribution of the training data used by G. Chryssolouris, M. Lee, and A. Ramsey. They present a new, or as they have termed it "a novel," method of computing confidence bounds on predictions from a feed-forward neural network with determined data structure. The authors [14] state that "this novel approach not only calculates the accuracy of the neural network but it also incorporates the influence of the training data density."

R.D. De Veaux, J.Schumi, J. Schweinsberg, and L.H. Ungar [13] state that it is possible to reduce the number of parameters needed for the calculation of confidence intervals by stopping the training algorithm prior to convergence of the network. The authors also claimed that the non-linear regression method for the calculation of confidence intervals is promising in theory, but it works poorly in practice. The authors presented a new technique of using weight 
decay, and they conclude by noting that using a weight decay constant as a regularizer, accurate function approximations are obtained.

L.Yang, T. Kavli, M. Carlin, S. Clausen, and P. F. M. de Groot [15] used non-linear regression and analyzed it over a 10-dimensional function with changing parameters, such as level of noise, the size of the training set, the number of training epochs, and by adding irrelevant inputs. They developed a method to estimate the bias of the coverage of confidence intervals. This bias then can be applied to correct the confidence bounds. However, they agreed that the standard non-linear regression confidence bound estimation algorithm gave satisfactory results.

S. L. Ho et. al. [41] applied a non-linear regression technique to estimate confidence bounds for time-series forecasting of a solder paste deposition process using neural networks. They ran simulation tests for two data sets with different nominal values (95\% and $90 \%)$, and they concluded that these confidence intervals gave satisfactory coverage percentages as compared to the nominal confidence levels.

The original algorithm for non-linear regression as a confidence interval estimator [16, $19,38-41]$ assumes that the distribution of the error for the neural network is normal. Neural networks can be considered as a non-linear system given as $f\left(\mathrm{x} ; \theta^{*}\right)$ with output $y$, where $\mathrm{x}$ is the set of inputs, $\theta^{*}$ represents the true values of the set of parameters, and $\theta$ for the function that models the system. The error $\varepsilon$ associated with the function in modeling the system is assumed to be independently and identically distributed with variance $\sigma^{2}$, where the distribution has the form $N\left(0, \sigma^{2}\right)$. With $n$ observations, Equation (10) represents the system:

$$
y_{i}=f\left(\mathrm{x}_{i} ; \theta^{*}\right)+\varepsilon_{i,} \quad i=1,2, \ldots, n .
$$

The least-squares estimate of $\theta^{*}$ is $\hat{\theta}$, which is obtained by minimizing the error function given in Equation (11). The backpropagation algorithm is used to minimize error function in neural networks. The predicted output from the model is $\hat{y}_{i}$, which can be represented in Equation (12).

$$
\begin{gathered}
S(\theta)=\sum_{i=1}^{n}\left[y_{i}-f\left(\mathrm{x}_{i} ; \theta\right)\right]^{2} \\
\hat{y}_{i}=f\left(\mathrm{x}_{i} ; \hat{\theta}\right)
\end{gathered}
$$


If the model is able to predict values close to the actual values, then $\hat{\theta}$ is close to the true value of the set of parameters $\theta^{*}$. A Taylor expansion is used to approximate $f(\mathrm{x} ; \hat{\theta})$ in terms of $f\left(\mathrm{x} ; \theta^{*}\right)$. This expansion is given in Equation (13).

$$
f\left(\mathrm{x}_{i} ; \hat{\theta}\right) \approx f\left(\mathrm{x}_{i} ; \theta^{*}\right)+\mathrm{f}_{0}^{T} .\left(\hat{\theta}-\theta^{*}\right)
$$

where,

$\mathrm{f}_{0}^{T}=\left(\frac{\partial f\left(\mathrm{x}_{i} ; \theta^{*}\right)}{\partial \theta_{1}^{*}}, \frac{\partial f\left(\mathrm{x}_{i} ; \theta^{*}\right)}{\partial \theta_{2}^{*}}, \ldots, \frac{\partial f\left(\mathrm{x}_{i} ; \theta^{*}\right)}{\partial \theta_{p}^{*}}\right)$ and $p$ is the number of parameters.

Using Equation (10) and (13), the difference between the true value of $y$ of the system and the predicted value $\hat{y}$ can be calculated as given in Equation (14). The expected value of this difference is given in Equation (15). The subscript value used in Equation (15) is to denote the set of points other than those used for the least-squares estimation of $\theta^{*}$.

$$
\begin{gathered}
\begin{array}{r}
y_{0}-\hat{y}_{0} \approx y_{0}-f\left(\mathrm{x}_{0} ; \theta^{2}\right)-\mathrm{f}_{0}^{T} \cdot\left(\hat{\theta}-\theta^{*}\right) \\
=\varepsilon_{0}-\mathrm{f}_{0}^{T} \cdot\left(\hat{\theta}-\theta^{*}\right)
\end{array} \\
E\left[y_{0}-\hat{y}_{0}\right] \approx E[\varepsilon 0]-\mathrm{f}_{0}^{T} E\left[\left(\hat{\theta}-\theta^{*}\right)\right] \approx 0 .
\end{gathered}
$$

The variance can be expressed as given in Equation (16) because of the statistical independence between $\hat{\theta}$ and $\varepsilon_{0}$.

$$
\operatorname{var}\left[y_{0}-\hat{y}_{0}\right] \approx \operatorname{var}\left[\varepsilon_{0}\right]+\operatorname{var}\left[\mathrm{f}_{0}^{T} \cdot\left(\hat{\theta}-\theta^{*}\right)\right] .
$$

For an error $\varepsilon_{0}$ with a normal distribution and with a mean of zero and a variance of $\sigma^{2}\left(N\left(0, \sigma^{2} \mathrm{I}_{n}\right)\right)$, the distribution of $\hat{\theta}-\theta^{*}$ can be approximated to have the distribution $N_{p}\left(0, \sigma^{2}\left[\mathrm{~F} .(\hat{\theta})^{T} \mathrm{~F} .(\hat{\theta})\right]^{-1}\right)$. The F. $(\hat{\theta})$ is the Jacobian matrix consisting of first derivatives in the form shown in Equation (17), where the single period denotes the matrix with first-order differential terms. 


$$
\mathrm{F} .(\hat{\theta})=\frac{\partial \mathrm{f}(\mathrm{x}, \hat{\theta})}{\partial \hat{\theta}}=\left[\begin{array}{cccc}
\frac{\partial \mathrm{f}_{1}\left(\mathrm{x}_{1}, \hat{\theta}\right)}{\partial \hat{\theta}_{1}} & \frac{\partial \mathrm{f}_{1}\left(\mathrm{x}_{1}, \hat{\theta}\right)}{\partial \hat{\theta}_{2}} & \cdots & \frac{\partial \mathrm{f}_{1}\left(\mathrm{x}_{1}, \hat{\theta}\right)}{\partial \hat{\theta}_{p}} \\
\frac{\partial \mathrm{f}_{2}\left(\mathrm{x}_{2}, \hat{\theta}\right)}{\partial \hat{\theta}_{1}} & \ldots & \ldots & \frac{\partial \mathrm{f}_{2}\left(\mathrm{x}_{2}, \hat{\theta}\right)}{\partial \hat{\theta}_{p}} \\
\vdots & \vdots & \vdots & \vdots \\
\frac{\partial \mathrm{f}_{n}\left(\mathrm{x}_{n}, \hat{\theta}\right)}{\partial \hat{\theta}_{1}} & \frac{\partial \mathrm{f}_{n}\left(\mathrm{x}_{n}, \hat{\theta}\right)}{\partial \hat{\theta}_{2}} & \ldots & \frac{\partial \mathrm{f}_{n}\left(\mathrm{x}_{n}, \hat{\theta}\right)}{\partial \hat{\theta}_{p}}
\end{array}\right]
$$

Equation 18 gives the unbiased estimator of $\sigma^{2}$ and using Equation (19), the Student $t$ distribution is given in Equation (20). Equation (21) gives the confidence interval $100^{*}(1-\alpha)$ for the predicted value $\hat{y}$.

$$
\begin{gathered}
s^{2}=\frac{\|\mathrm{y}-\mathrm{f}(\mathrm{x}, \hat{\theta})\|^{2}}{n-p} \\
t_{n-p} \sim \frac{y_{0}-\hat{y}_{0}}{\sqrt{\operatorname{var}\left[y_{0}-\hat{y}_{0}\right]}} \approx \frac{y_{0}-\hat{y}_{0}}{\sqrt{s^{2}+s^{2} \mathrm{f}_{0}^{T}\left(\mathrm{~F}^{T} \cdot \mathrm{F} .\right)^{-1} \mathrm{f}_{0}}} \approx \frac{y_{0}-\hat{y}_{0}}{s\left(1+\mathrm{f}_{0}^{T}\left(\mathrm{~F}^{T} \cdot \mathrm{F} .\right)^{-1} \mathrm{f}_{0}\right)^{\frac{1}{2}}} \\
\hat{y}_{0} \pm t_{n-p}^{\alpha / 2} s\left(1+\mathrm{f}_{0}^{T}\left(\mathrm{~F}^{T} \cdot \mathrm{F} .\right)^{-1} \mathrm{f}_{0}\right)^{\frac{1}{2}}
\end{gathered}
$$

Rivals and Personnaz [18, 39, and 40] use the least squares estimation term instead of NLR. The calculation of the inverse of the matrix $F^{T} F$ is a known problem in the literature, as the values of the matrix $F$ nearly become singular. Rivals and Personnaz $[39,40]$ provide an alternate solution as discussed below.

Rivals and Personnaz mention that a statement on rank deficiency of $\mathrm{F}$ cannot be made. The estimation of whether confidence interval can be estimated accurately or not should be decided on the need of inverse of $\mathrm{F}^{\mathrm{T}} \mathrm{F}$. The most accurate estimation was performed using F's singular value decomposition (SVD) $\mathrm{F}=\mathrm{U} \Sigma \mathrm{V}^{\mathrm{T}}$. They provided equations to obtain the computer version of $\left(\mathrm{F}^{\mathrm{T}} \mathrm{F}\right)^{-1}$ in the form of Equation (22). 


$$
\widehat{\left(F^{T} F\right)^{-1}}=\widehat{V}\left(\sum^{T} \sum\right)^{-1} \widehat{V}^{T} \text { with } \begin{cases}\sqrt{\left.\left(\sum^{T} \sum\right)^{-1}\right]_{i i}}=\frac{1}{\left(\widehat{\sigma_{i}}\right)^{2}} & \text { for } i=1 \text { to } q \\ {\left[\widehat{\left.\left(\sum^{T} \sum\right)^{-1}\right]_{i j}}=0\right.} & \forall i \neq j\end{cases}
$$

where $q$ ( $p$ as used in G. Chryssolouris [16]) is the number of parameters a neural network has.

The absolute error on the $i^{\text {th }}$ singular value is bounded by Equation (23).

$$
\left|\sigma_{i}-\hat{\sigma}_{i}\right| \leq \sigma_{1} \varepsilon \text { for } i=1 \text { to } q
$$

where $\varepsilon$ is the computer unit rounded off ( $\varepsilon=10^{-16}$ for usual computers).

The relative error on the smallest singular values is bounded by Equation (24).

$$
\left|\frac{\sigma_{q}-\widehat{\sigma_{q}}}{\sigma_{q}}\right| \leq \frac{\sigma_{1}}{\sigma_{q}} \varepsilon=\kappa(F) \varepsilon
$$

It can be seen that the absolute error was directly related to the condition number $\kappa(F)$ of $F$, which is the ratio of its largest to its smallest singular value. The relative error on the smallest singular value may be $100 \%$ if $\kappa(F)$ reaches $1 / \varepsilon=10^{16}$.

Therefore, Rivals and Personnaz recommend discarding neural networks with $\widehat{\kappa(F)}>$ $10^{8}$ as Equation (22) involves calculating the inverse of the squared Jacobian matrix, and hence, the inverse of the computed squared singular values. The inverse of the matrix $\left(F^{T} F\right)$ can only be calculated with precision when $\widehat{\kappa(F)}<<10^{8}$. Also, the precision of $\kappa(F)$ itself is excellent when $\widehat{\kappa(F)}$ is below $10^{8}$.

The rank of Jacobian matrix can be estimated as the number of computer singular values that are larger than the threshold of $\widehat{\sigma_{1}} \varepsilon$, as given in Equation (25).

$$
\hat{r}=\operatorname{card}\left\{\widehat{\sigma_{i}}>\widehat{\sigma_{1}} \varepsilon, i=1 \text { to } q\right\}
$$

Rivals and Personnaz suggest an alternate way to calculate the inverse than Equation(22), which does not deal with the computation of the inverse of the Jacobian matrix. When $r=q$, the diagonal elements of the $H=F\left(F^{T} F\right)^{-1} F^{T}$ can be calculated using Equation (26). 


$$
\widehat{h_{k k}}=\sum_{i=1}^{\hat{r}}\left(\widehat{u_{k i}}\right)^{2} \text { for } k=1 \text { to } N
$$

where $\hat{r}$ is calculated using Equation (25).

As Equation (26) does not require the inverse of the square of possibly inaccurate small singular values, it is less sensitive to the ill-conditioning of $F$ than $\left(F^{T} F\right)^{-1}$.

Two additional tests are performed on values which are of interest for the computation of CI's and are related to the orthogonal projection matrix $H$ on the range of $F$. If $F$ is of full rank, since $H$ is a projection matrix, Equation (27) and (28) should be satisfied.

$$
\begin{gathered}
\operatorname{trace}(H)=\sum_{k=1}^{N} h_{k k}=\operatorname{rank}(H)=q \\
\frac{1}{N} \leq h_{k k} \leq 1 \text { for } k=1 \text { to } N
\end{gathered}
$$

Rivals and Personnaz mentioned the importance of verifying Equation (27) and (28), since for an input $x^{k}$, belonging to the training set, the expression of the CI is precisely given by Equation (29), rather than Equation (21).

$$
\widehat{y_{k}} \pm t_{N-q}^{\alpha} s \sqrt{h_{k k}}
$$

They caution on the usefulness of the CIs ."If (27) or (28) are not satisfied, even if the network has not been diagnosed to overfit by a test on the singular values, the computation of the CIs is meaningless." All cases studied in this research satisfied both the conditions.

Although, this model assumes that error associated with modeling is normally distributed, Shao et. al. [14] mention the importance of including actual error in the confidence intervals, as the width of the confidence intervals is in proportion to the standard deviation of the error vector. Therefore, Shao et. al. suggest multiplying the CIs by the ratio of the prediction error to the standard deviation of the whole vector by $\lambda$. This correction factor can be calculated using Equation (30).

$$
\lambda_{i}=\frac{\left|y_{i}-\hat{y}_{i}\right|}{\operatorname{std}\left(\left|y_{i}-\hat{y}_{i}\right|\right)}, i=1,2, \cdots n
$$

where $\mathrm{n}$ is the number of predicted points. 
Thus, the modified confidence intervals can be given by Equation

$$
\hat{y}_{k} \pm t_{N-q}^{\alpha} \lambda_{k} s \sqrt{h_{k k}}
$$

Since the paper published by G. Chryssolouris [16], various researchers studied nonlinear regression to calculate the confidence intervals on the output of neural networks. Coverage probability (CP) was used as a tool to test the accuracy of this estimation by L. H. Ungar [17]. Rivals and Personnaz [18] reported a different way to estimate the calculation terms as the method reported by G. Chryssolouris [16] created problems in the calculations.

\subsubsection{Bootstrap Estimation}

A book by Efron and Tibshirani on bootstrapping [20] affirms that confidence interval can be obtained by using a resampling technique such as bootstrapping. T. Heskes [30] states that bootstrapping is based on the idea that the available data set is nothing but a particular realization of some unknown probability distribution. Instead of sampling over the true distribution, which is obviously impossible, an empirical distribution is defined, according to Heskes [30].

The bootstrap is a computer-based method for assigning measures of accuracy to statistical estimates. Bootstrapping will provide a nonparametric confidence interval for any population estimate. This process involves creating a large number of bootstrap samples by repeatedly resampling (with replacement) the original dataset in a random order, each with probability of $1 / n$, where $n$ is the number of samples. By resampling with replacement it, is indicated that the same data row can be used repeatedly to generate a bootstrap sample if a random number matching that row is selected. Each bootstrap sample is used as a training set. An illustration on bootstrap resampling is provided in Table 2.2 and the data used are taken from McKim [46] (also appeared in K. N. Lankalpalli and R. C. Creese [21]). To illustrate bootstrap sampling with replacement, two bootstrap samples are generated by creating random number between one and 23 as shown in Table 2.2. 
Table 2.2 Bootstrap Resampling Example [46]

\begin{tabular}{|c|c|c|c|c|c|c|c|c|c|c|c|}
\hline & \multicolumn{2}{|c|}{ Original Data } & \multicolumn{3}{c|}{ Bootstrap Sample Set 1 } & \multicolumn{3}{c|}{ Bootstrap Sample Set 2 } \\
\hline & Flow & Head & $\begin{array}{c}\text { Actual } \\
\text { Price }\end{array}$ & $\begin{array}{c}\text { Random } \\
\text { Number }\end{array}$ & Flow & Head & $\begin{array}{c}\text { Actual } \\
\text { Price }\end{array}$ & $\begin{array}{c}\text { Random } \\
\text { Number }\end{array}$ & Flow & Head & $\begin{array}{c}\text { Actual } \\
\text { Price }\end{array}$ \\
\hline S.N. & gpm & inches & $\mathbf{\$}$ & & gpm & inches & $\$$ & & Gpm & inches & $\$$ \\
\hline 1 & 100 & 700 & 25,584 & 9 & 300 & 2000 & 36,660 & 19 & 600 & 700 & 31,668 \\
\hline 2 & 100 & 1200 & 28,296 & 22 & 700 & 700 & 43,668 & 12 & 400 & 1200 & 31,860 \\
\hline 3 & 100 & 2000 & 32,160 & 20 & 600 & 1200 & 34,008 & 19 & 600 & 700 & 31,668 \\
\hline 4 & 200 & 700 & 25,584 & 21 & 600 & 2000 & 39,240 & 11 & 400 & 700 & 28,908 \\
\hline 5 & 200 & 1200 & 28,296 & 18 & 500 & 2400 & 39,240 & 5 & 200 & 1200 & 28,296 \\
\hline 6 & 200 & 2000 & 32,160 & 18 & 500 & 2400 & 39,240 & 11 & 400 & 700 & 28,908 \\
\hline 7 & 300 & 700 & 27,924 & 23 & 700 & 1200 & 34,008 & 12 & 400 & 1200 & 31,860 \\
\hline 8 & 300 & 1200 & 30,936 & 16 & 500 & 1200 & 31,860 & 10 & 300 & 2400 & 39,240 \\
\hline 9 & 300 & 2000 & 36,660 & 8 & 300 & 1200 & 30,936 & 2 & 100 & 1200 & 28,296 \\
\hline 10 & 300 & 2400 & 39,240 & 7 & 300 & 700 & 27,924 & 7 & 300 & 700 & 27,924 \\
\hline 11 & 400 & 700 & 28,908 & 14 & 400 & 2400 & 39,240 & 8 & 300 & 1200 & 30,936 \\
\hline 12 & 400 & 1200 & 31,860 & 14 & 400 & 2400 & 39,240 & 19 & 600 & 700 & 31,668 \\
\hline 13 & 400 & 2000 & 36,660 & 16 & 500 & 1200 & 31,860 & 17 & 500 & 2000 & 36,660 \\
\hline 14 & 400 & 2400 & 39,240 & 2 & 100 & 1200 & 28,296 & 16 & 500 & 1200 & 31,860 \\
\hline 15 & 500 & 700 & 28,908 & 5 & 200 & 1200 & 28,296 & 7 & 300 & 700 & 27,924 \\
\hline 16 & 500 & 1200 & 31,860 & 16 & 500 & 1200 & 31,860 & 18 & 500 & 2400 & 39,240 \\
\hline 17 & 500 & 2000 & 36,660 & 17 & 500 & 2000 & 36,660 & 9 & 300 & 2000 & 36,660 \\
\hline 18 & 500 & 2400 & 39,240 & 9 & 300 & 2000 & 36,660 & 13 & 400 & 2000 & 36,660 \\
\hline 19 & 600 & 700 & 31,668 & 22 & 700 & 700 & 43,668 & 2 & 100 & 1200 & 28,296 \\
\hline 20 & 600 & 1200 & 34,008 & 23 & 700 & 1200 & 34,008 & 14 & 400 & 2400 & 39,240 \\
\hline 21 & 600 & 2000 & 39,240 & 10 & 300 & 2400 & 39,240 & 22 & 700 & 700 & 43,668 \\
\hline 22 & 700 & 700 & 43,668 & 15 & 500 & 700 & 28,908 & 2 & 100 & 1200 & 28,296 \\
\hline 23 & 700 & 1200 & 34,008 & 5 & 200 & 1200 & 28,296 & 5 & 200 & 1200 & 28,296 \\
\hline
\end{tabular}

Baxt and White [22] have successfully used bootstrapping to compute confidence intervals for clinical input variable effects in a network to identify the presence of acute myocardial infarction. They took 19 input variables and one binary output variable, which would predict if a patient has myocardial infarction. The authors generated 1000 sets of 706 input-target pairs. After training these 1000 sets using backpropagation, they found the sample means and examined the network statistically. The authors claimed that they are the first to statistically validate observed relationships between the input information processed by an ANN and its target. They also claim that bootstrapping is not limited by network architecture and is applicable to most network constructs. They found that one of the indicators or input variables of infarction previously identified as statistically significant was in fact statistically insignificant. 
G. Papadopoulos et. al. [23], R. Tibshirani [24], and R. Dybowski et. al. [25] discuss the use of bootstrapping to calculate confidence intervals for neural networks. G. Papadopoulos simulated three artificial data sets 120 input-target pairs for training) and one real data set (369 input-target pairs for training) [23]. Moreover, the authors added noisy data, which had been treated as a function of the inputs. The number of bootstrap samples used in G. Papadopoulos et.al. [23] was 20. Tibshirani [24] states that the number of bootstrap samples or number for retraining the network is typically between 20 and 200, but he used 20 samples. The data set used in [24] had 111 input-target pairs or observations. The author concluded that the bootstrap methods provided the most accurate estimates of the standard errors of the predicted value.

Bhide and Piovoso [42] studied the bootstrap technique for generating appropriate statistic for the confidence interval on the output of the neural networks. Also, W. Y. Goh et.al. [43] used the bootstrap estimation technique to calculate the confidence intervals using recurrent neural networks. The bootstrap pairs sampling algorithm [24] is given as follows:

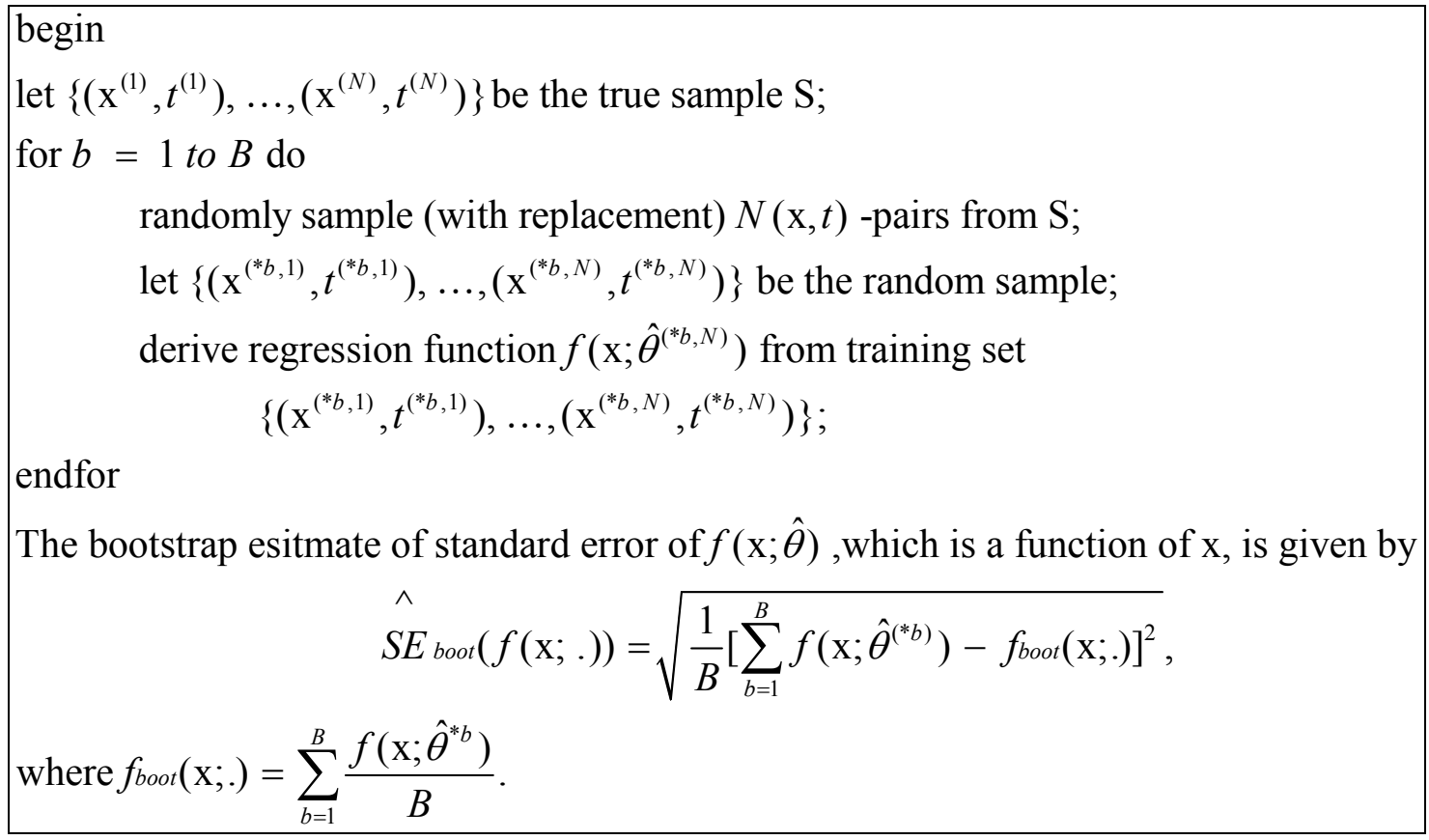

Assuming a normal distribution for $f(\mathrm{x} ; \hat{\theta})$ over the space of all possible $\hat{\theta}$, the $95 \%$ bootstrap confidence interval for $f(\mathrm{x})$ can be given in Equation (32).

$$
f(\mathrm{x} ; \hat{\theta}) \pm t_{0.025,[B]} \cdot \widehat{S E}_{\text {boot }}(f(\mathrm{x} ; .))
$$


Using bootstrapping, a realization of some unknown probability distribution of the data and a nonparametric confidence interval for any population estimate was found.

\subsubsection{Maximum Likelihood (ML) Estimation}

Dybowski and Roberts in [25] discuss the idea of using maximum likelihood estimators (MLE) for the calculation of confidence bounds. The definition of maximum likelihood estimation as given in R.E. Walpole et.al. [26] as follows.

"Given independent observations $x_{1}, x_{2}, \ldots, x_{n}$ from a probability density function (continuous case) or probability mass function (discrete case) $f(x, \theta)$ the maximum likelihood estimator $\hat{\theta}$ is that which maximizes the likelihood function

$$
L\left(x_{1}, x_{2}, \ldots, x_{n}, \theta\right)=f\left(x_{1}, \theta\right) \cdot f\left(x_{2}, \theta\right) \cdot \ldots \cdot f\left(x_{n}, \theta\right) "
$$

For discrete cases, the maximum likelihood estimator is one that results in a maximum value of joint probability or maximizes the likelihood of the sample. The notion of maximum likelihood extends to the estimation of parameters of continuous distribution too. Differentiating the probability density or mass function and setting the derivative to zero should give the maximum value.

G. Papadopoulos et. al.[25], state the methodology for incorporating the maximum likelihood estimation for neural networks. They say if a feed-forward neural network $f(\mathrm{x} ; \hat{\theta})$ is trained on a dataset $\left\{\mathrm{x}^{(1)}, t^{(1)}, \ldots, \mathrm{x}^{(N)}, t^{(N)}\right\}$ by minimizing $\operatorname{Err}(\theta)$ (given in Equation (34)), where $\theta$ are the network weights, it can be shown that the resulting network approximates the mean value for $t$ conditioned $\mathrm{x}$,

$$
f(\mathrm{x} ; \hat{\theta}) \approx E[t \mid \mathrm{x}]
$$

the approximation becoming equality if $N$ goes to infinity and $f(\mathrm{x} ; \hat{\theta})$ has unlimited flexibility. Therefore, a feed-forward network trained via $\operatorname{Err}(\theta)$ can be regarded as a regression function.

$$
\operatorname{Err}(\theta)=\frac{1}{2} \sum_{n=1}^{N}\left[f\left(\mathrm{x}^{(n)} ; \hat{\theta}\right)-t^{(n)}\right]^{2}
$$

The variance of $t$ associated with output $f(\mathrm{x} ; \hat{\theta})$ is given, as in Equation (35). A second neural network $\hat{\sigma}^{2}(\mathrm{x} ; \mathrm{u})$ using squared residuals $(f(\mathrm{x} ; \hat{\theta})-t)^{2}$ as the target values for the sum-ofsquares function given in Equation (36) so that $\operatorname{Var}[t \mid \mathrm{x}] \approx \hat{\sigma}^{2}(\mathrm{x} ; \hat{\mathrm{u}})$. 


$$
\begin{gathered}
\operatorname{Var}[t \mid \mathrm{x}]=E\left[(f(\mathrm{x} ; \hat{\theta})-t)^{2} \mid \mathrm{x}\right] . \\
\frac{1}{2} \sum_{n=1}^{N}\left\{\hat{\sigma}^{2}\left(\mathrm{x}^{(n)} ; \mathrm{u}\right)-\left[f\left(\mathrm{x}^{(n)} ; \hat{\theta}\right)-t^{(n)}\right]^{2}\right\}^{2}
\end{gathered}
$$

By assuming a Gaussian distribution for $t$, given in Equation (37), the confidence interval for $t$ about $f(\mathrm{x} ; \hat{\theta})$ can be obtained.

$$
p(t \mid \mathrm{x})=\frac{1}{\sqrt{2 \pi \hat{\sigma}(\mathrm{x} ; \hat{\mathrm{u}})}} \exp \left\{\frac{-(f(\mathrm{x} ; \hat{\theta})-t)^{2}}{2 \hat{\sigma}^{2}(\mathrm{x} ; \hat{\mathrm{u}})}\right\}
$$

By training a separate neural network for the error terms, the variance on the predicted output was estimated and hence the confidence intervals were computed.

\subsection{Summary}

The literature search provided important views in the existing research in the area of confidence interval computation. It was noted that even though this area is relatively new, various researchers are attempting to develop with better techniques or modifications. The primary techniques for constructing confidence intervals existing in the literature were found to be non-linear regression, bootstrap algorithm, maximum-likelihood, and approximate Bayesian approach.

The researchers used different approaches with various data sets and parameters, leaving little ground for direct comparisons. Although some researchers were able to tell which techniques were actually better than others in some aspects, no author was able to recommend a single approach applicable to any condition. Artificial neural networks are commonly known as "black boxes" and are not able to explain the results and give assurances on the results. However, many authors in the literature claim that confidence intervals can be constructed on the results of neural networks under specified assumptions.

\subsection{Research Objective}

The primary objective of the research was to model, compare, and analyze non-linear regression, bootstrapping, and MLE in the literature on constructing confidence intervals for the outputs of neural networks. As mentioned earlier, it is very important to have confidence intervals on the outputs of the "black box" model. However, this field of calculating confidence 
intervals on the outputs of neural networks is relatively new and research is an ongoing process. Therefore, it is imperative that the existing methods of calculating the confidence intervals should be tested and analyzed as much as possible on different scenarios.

This research was conducted to explore different options on constructing the confidence intervals available in the literature. Even though the literature provides some insight about these techniques, a more detailed and organized study was required. This research attempted to provide an easy-to-use neural network model for any application with confidence intervals on the output. 


\section{Model Development}

\subsection{Development of an Artificial Neural Network Model}

An artificial neural network model with feed-forward architecture was developed using backpropagation algorithm for updating the weights developed by Laurene Fausett [5]. The backpropagation algorithm can be depicted in Figure 3.1. The number of hidden layers and output neurons were limited to one for ease of calculation.

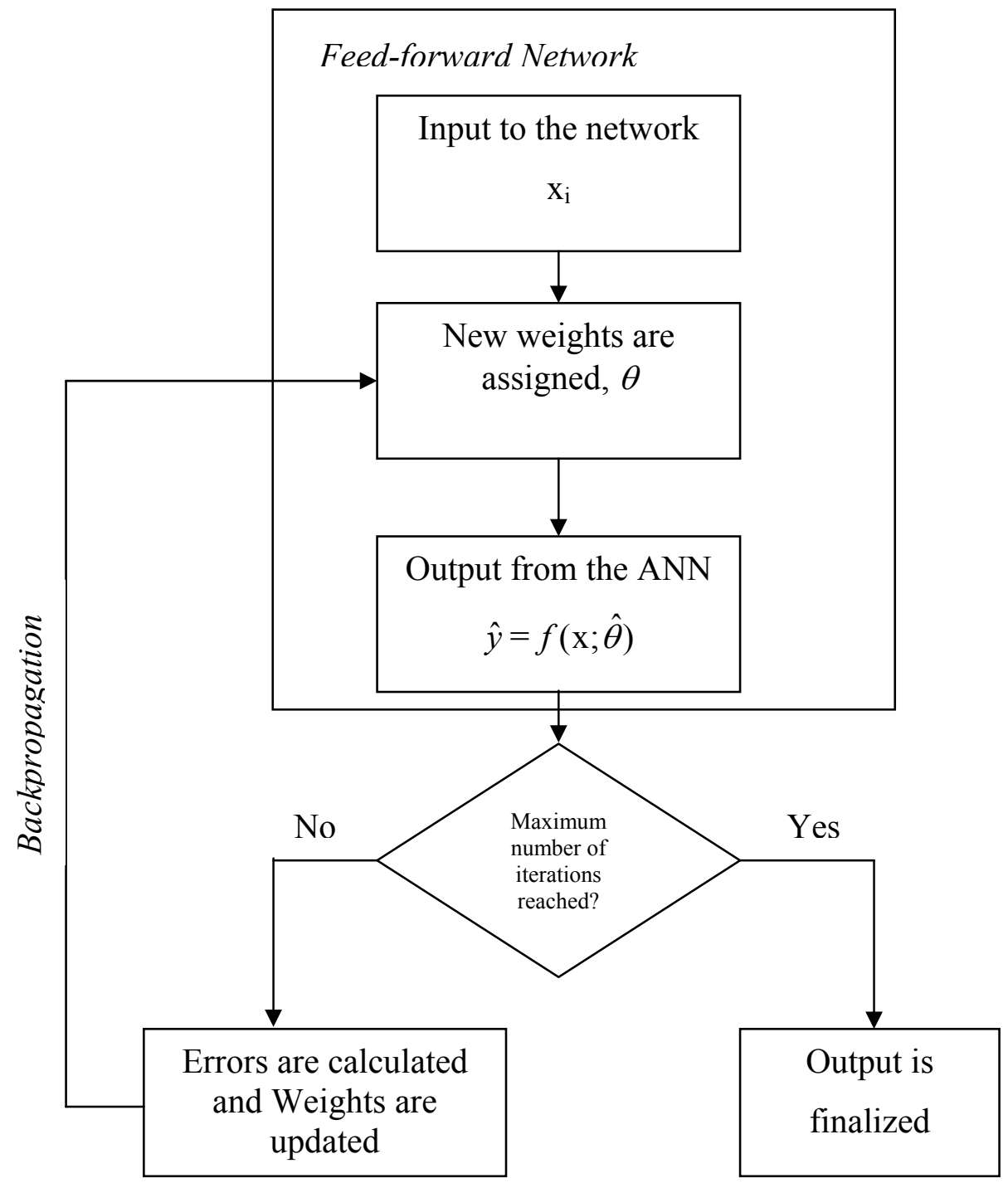

Figure 3.1 Flowchart of the Backpropagation Algorithm 
Equation (38) was used to generate a data set of 250 points with $\mathrm{X}$ being randomly selected between 0 and 1 . There is no recommended rule for selecting the number of neurons in the hidden layer, but they are generally selected more than the number of input parameters. Too many hidden neurons may result in a failure to generalize properly, and too few hidden neurons may result in a failure to achieve a low average error. Therefore, the number of hidden neurons should be chosen after some trial and error. For this specific problem, the number of neurons in the hidden layer was selected as two because the problem was not complex.

$$
y=2 X_{1}^{2}+3 X_{2}^{3}+4 X_{3}+X_{4}^{1 / 2}+4 X_{5}
$$

The stopping condition generally used is either based on the Mean Square Error (MSE) or the number of iterations. The number of iterations, also called as epochs, is the number of times the error is back propagated and weights are updated. The flexible number of iterations helps to avoid the problem of overfitting, which means that the model has become too powerful to model the associated noise with the data. The scaling of the input and output variables was necessary as the sigmoidal activation function was used which limits the values between zero and one.

\subsubsection{Scaling}

The binary sigmoidal activation function, $y=1 /\left(1+\exp \left(-x_{i}\right)\right)$, ensures that the output of any neuron is between 0 and 1, which causes the problem of restricting the output to between 0 and 1, when the actual output may not be in that range. Researchers came up with this idea of scaling the input data set that can be used by the neural network and the output must be scaled back to give the output in the original comparable form. The data used in this research were scaled between 0.1 and 0.9 using Equation (39), as given in [21] and scaling back of the data was done by using Equation (40).

$$
v_{\text {acaled }}=\frac{0.8 * \text { val }_{\text {org }}+0.1 * v a l_{\max }-0.9 * v a l_{\text {min }}}{v a l_{\max }-v a l_{\text {min }}}
$$

where,

$v a l_{\text {scaled }}=$ value to be scaled

$v a l_{\text {org }}=$ original value

$v a l_{\max }=$ maximum value of the same data row of the value to be scaled

$v a l_{\min }=$ minimum value of the same data row of the value to be scaled 


$$
v a l_{\text {new }}=\frac{0.9 * v a l_{\min }-0.1 * v a l_{\max }+v a l_{\text {saled }} *\left(v a l_{\max }-v a l_{\min }\right)}{0.8}
$$

where,

$v a l_{\text {new }}=$ Value to be scaled back

For example, if we have to scale the first value (100) from Table 2.2, it would be done as given in Equation (41). The maximum and minimum values in the data set are 700 and 100 respectively. If the value to be scaled is the minimum value of that data set, the scaled value is 0.1 , which is the minimum value of the scale $(0.1,0.9)$.

$$
\text { val }_{\text {saled }}=\frac{0.8 * 100+0.1 * 700-0.9 * 100}{700-100}=0.1
$$

\subsubsection{Neural Network Model Results}

From the 250 data sets generated using Equation(38), 175 were used for training and the remaining 75 were used for testing the network. The data used are presented in Appendix A. Using two neurons in the hidden layer and 20, 30, and 40 epochs, the network was trained and the results are shown in Figure 3.2. The $\mathrm{R}^{2}$ between the actual and model output for training data obtained were $0.31,0.63$, and 0.96 for 20,30 , and 40 iterations, respectively. It can be seen that the $\mathrm{R}^{2}$ value increases with an increase in the number of iterations or epochs, and therefore, there is a decrease in MSE with an increase in the number of iterations. However, it should be noted that the number of epochs may not necessarily reduce the error in the predicted values as it also depends on the starting random weights.

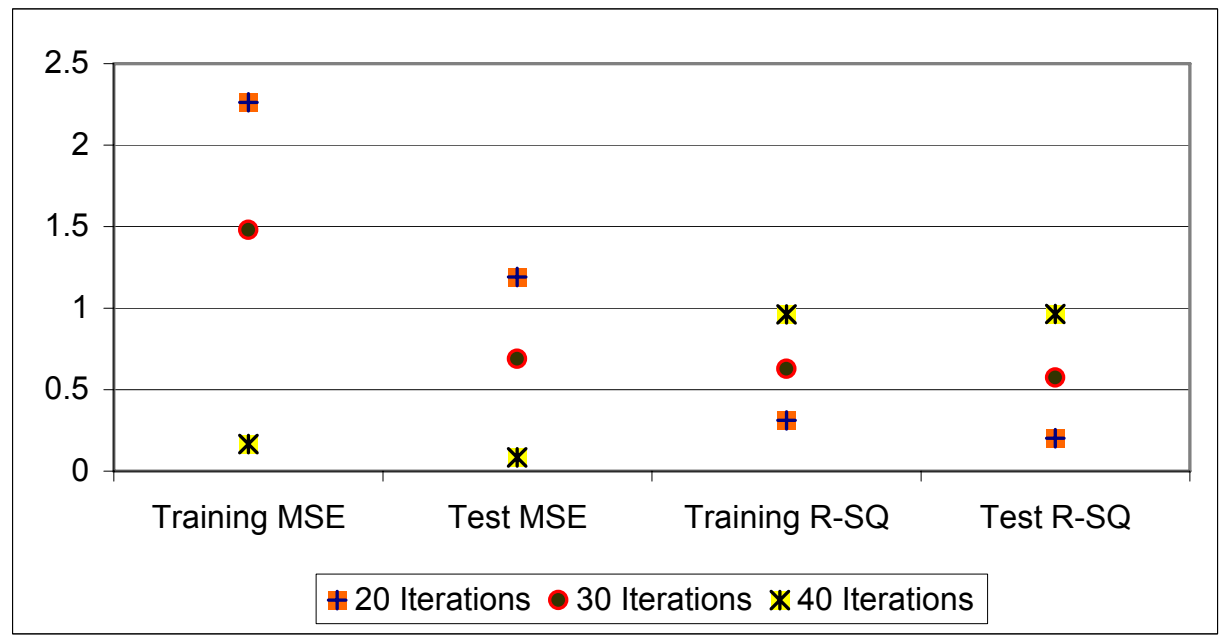

Figure 3.2 MSE for Training and Test Data, and R-square for Training and Test Data Using 20, 30, and 40 Epochs 


\subsubsection{Random Weight Initialization}

Although the usual practice is to assign small random numbers as starting weights, D. Nguyen and B. Widrow [44] developed a new algorithm, known as the Nguyen-Widrow initialization (NWI) method. It is a simple modification of the common random weight initialization, which allows for gives much faster learning by the neural network. This initialization of weights was used throughout the research.

The approach is based on a geometrical analysis of the response of the hidden neurons to a single input; the analysis is extended to the case of several inputs by using Fourier transformations. The weights from hidden units to the output units and biases on the output units are initialized to random values between -0.5 and 0.5 .as is commonly the case.

This initialization of the weights from input to output units is done to improve the hidden units' ability to learn. This is accomplished by distributing the initial weights and biases so that for each input pattern, it is likely that the net input to one of the hidden units will be in the range in which that hidden neuron will learn most readily. This method is described as follows:

$n \quad$ number of input units

$p \quad$ number of hidden units

$\beta \quad$ scale factor, given as:

$$
\beta=0.7(p)^{1 / n}=0.7 \sqrt[n]{p}
$$

The procedure consists of the following steps:

For each hidden unit $(j=1, \ldots, p)$ :

Initialize its weight vector (from the input units):

(old) $=$ random number between -0.5 and 0.5 (or between $-\gamma$ and $\gamma$ ).

Compute $\quad \| V_{i j}($ old $) \|=\sqrt{V_{1 j}(\text { old })^{2}+V_{2 j}(\text { old })^{2}+\ldots+V_{n j}(\text { old })^{2}}$

Reinitialize weights:

$$
v_{i j}=\frac{\beta v_{i j}(\text { old })}{\| V_{i j}(\text { old }) \|}
$$

set- bias: 


$$
v_{0 j}=\text { random number between }-\beta \text { and } \beta
$$

A neural network was tested by using the Nguyen-Widrow initialization method and without using it. Data from McKim [46] was used for this test. Ten simulations were run with the parameters given in Table 3.1. Mean squared errors (MSE) for these simulations are shown in Table 3.2 and Figure 3.3.

Table 3.1 Parameters for Neural Network to Test Nguyen-Widrow Initialization

\begin{tabular}{|l|c|}
\hline Hidden Neurons & 2 \\
\hline Iterations & 50 \\
\hline Activation function (Input-Hidden) & Sigmoid \\
\hline Activation function (Hidden-Output) & Linear \\
\hline Learning rate & 0.2 \\
\hline Observations for training & 19 \\
\hline Observations for test & 4 \\
\hline
\end{tabular}


Table 3.2 Mean Squared Error (MSE) with and without Nguyen-Widrow Initialization

\begin{tabular}{|c|c|c|}
\hline $\begin{array}{c}\text { Simulation } \\
\text { Number }\end{array}$ & $\begin{array}{c}\text { MSE with-NWI } \\
\text { (Millions) }\end{array}$ & $\begin{array}{c}\text { MSE without-NWI } \\
\text { (Millions) }\end{array}$ \\
\hline 1 & 161 & 566 \\
\hline 2 & 220 & 340 \\
\hline 3 & 172 & 480 \\
\hline 4 & 183 & 540 \\
\hline 5 & 137 & 355 \\
\hline 6 & 348 & 290 \\
\hline 7 & 161 & 434 \\
\hline 8 & 307 & 362 \\
\hline 9 & 210 & 431 \\
\hline 10 & 359 & $\mathbf{4 1 4}$ \\
\hline Average & $\mathbf{2 2 6}$ & $\mathbf{2 9 0 - 5 6 6}$ \\
\hline Range & $\mathbf{1 3 7 - 3 5 9}$ & \\
\hline
\end{tabular}

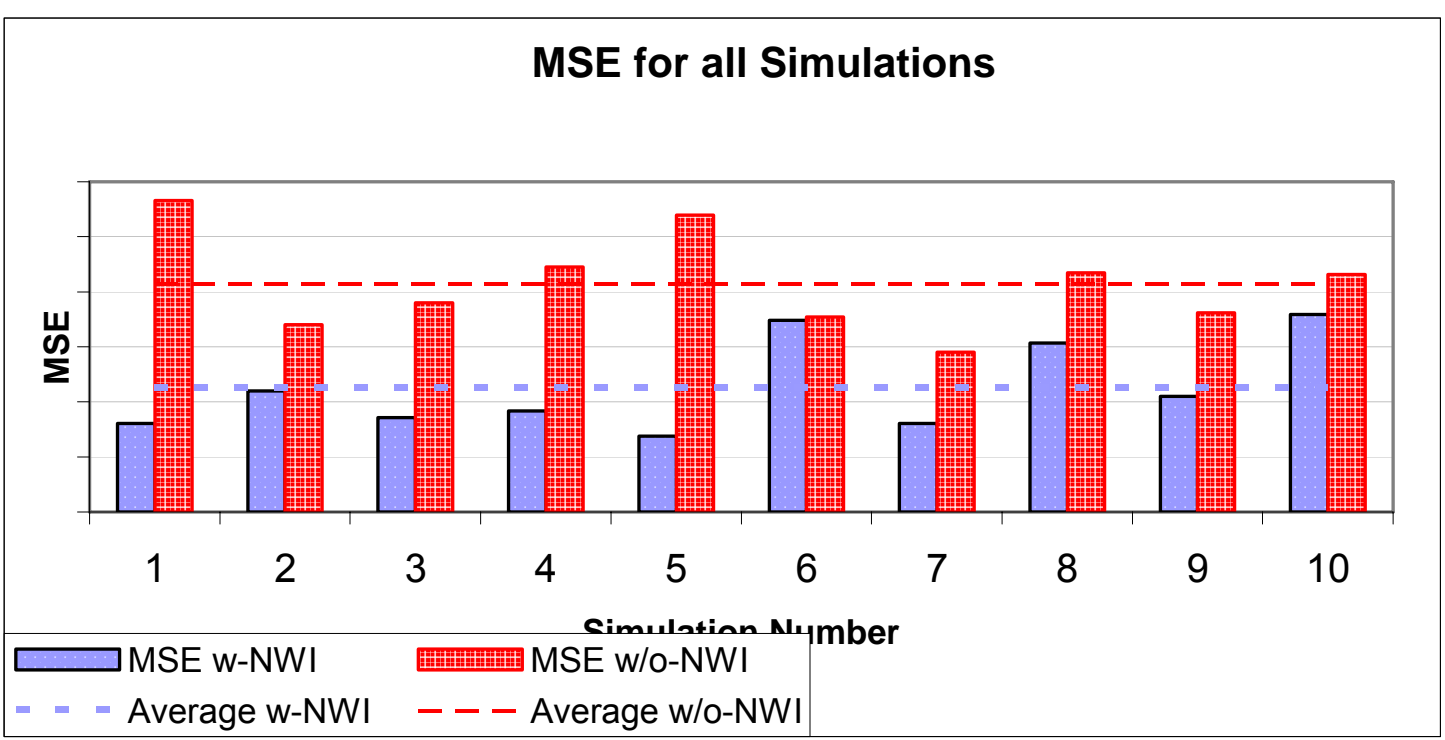

Figure 3.3 MSE and Average MSE for Neural Networks with and without NWI 
It can be seen from Table 3.2 and Figure 3.3 that neural network with NWI outperform the neural network without NWI. In only one of the ten cases were the MSE values approximately equal. The average MSE for the neural networks with NWI is $45 \%$ lower than the average MSE for the neural networks without NWI. A brief manual of this NN program is given in Appendix E.

\subsection{Development of Confidence Interval for Linear Regression}

A simple linear regression model is given in Equation (42).

$$
Y_{i}=\beta_{0}+\beta_{1} X_{i}+\varepsilon_{i}
$$

where,

$Y_{i}$ is the dependent or response variable in the $i^{t h}$ trial $(i=1, \ldots, \mathrm{n})$

$X_{i}$ is the independent variable

$\beta_{0}$ and $\beta_{1}$ are parameters to be estimated

$\varepsilon_{i}$ is the normally distributed error term with expected value to be zero and variance $\sigma^{2}$

The parameters $\beta_{0}$ and $\beta_{1}$ are estimated by minimizing the sum of the squared

residuals as given in Equation (43).

$$
\sum_{i=1}^{n} e_{i}^{2}=\sum_{i=1}^{n}\left(Y_{i}-\hat{Y}_{i}\right)^{2}
$$

where $\widehat{Y}_{i}$ is the predicted value using estimated parameters $\beta_{0}$ and $\beta_{1}$. The MSE or error mean square given by Equation (44) is an unbiased indicator of $\sigma^{2}$.

$$
M S E=\frac{\sum_{i=1}^{n} e_{i}^{2}}{n-2}
$$

Confidence intervals for linear regression are based on the estimated variance of $\hat{Y}_{h}$, the predicted value for observation $h$ [45]. This variance is given by Equation (45).

$$
\sigma^{2}\left\{\widehat{Y}_{h}\right\}=\sigma^{2}\left[\frac{1}{n}+\frac{\left(X_{h}-\bar{X}\right)^{2}}{\sum\left(X_{i}-\bar{X}\right)^{2}}\right]
$$

When MSE is substituted for $\sigma^{2}$, the estimated variance of $\hat{Y}_{h}, \mathrm{~s}^{2}\left\{\hat{Y}_{h}\right\}$, can be calculated by Equation (46). 


$$
s^{2}\left\{\widehat{Y}_{h}\right\}=\operatorname{MSE}\left[\frac{1}{n}+\frac{\left(X_{h}-\bar{X}\right)^{2}}{\sum\left(X_{i}-\bar{X}\right)^{2}}\right]
$$

Confidence intervals with 1- $\alpha$ confidence for $\mathrm{E}\left\{\hat{Y}_{h}\right\}$ can be calculated by using Equation(47), and the confidence interval for individual observation is given by Equation (48).

$$
\begin{gathered}
\widehat{Y}_{h} \pm t_{1-\alpha / 2}^{n-2} S\left\{\widehat{Y}_{h}\right\} \\
\widehat{Y}_{h} \pm t_{1-\alpha / 2}^{n-2}\left[S^{2}\left\{\widehat{Y}_{h}\right\}+M S E\right]^{1 / 2}
\end{gathered}
$$

\subsubsection{Linear Regression Confidence Intervals for Tree Data Set [46]}

The tree data set obtained from SAS's website [47] had 104 rows and three columns. The three columns (variables) were rootstock, trunk girth, and weight of the tree, and the data is in Table A-2. In the 1930's, the weights and trunk girths were measured for eight specimens from each of thirteen rootstocks for a total of 104 tree specimens. These data were collected to determine if the girth and/or rootstock of the trees were useful in predicting the weight of trees. As the rootstock was not a continuous variable, it was excluded from this research. This data table can be found in Table A-2. Out of 104 observations, 80 were used for training and 24 for testing. Regression summary and the ANOVA table on these data are given in Table 3.3.

The estimated variance $s^{2}\left\{\widehat{Y}_{h}\right\}$ for the first observation $\left(X_{\mathrm{h}}=406\right)$ in e Figure 3.2 was calculated using Equation 46. Substituting $\mathrm{MSE}=29,644, \mathrm{n}=80, \bar{X}=395$ and $\sum\left(X_{i}-\bar{X}\right)^{2}=376,758$ in Equation(46), $s^{2}\left\{\widehat{Y}_{h}\right\}=380.22$ was obtained. The confidence intervals for mean and confidence intervals for the individual observations were calculated using Equation (47) and (48) respectively, as shown in Figure 3.2 with $t_{0.975}^{78}=1.665$.

Using the coefficients found during the regression analysis, predicted values were calculated. The predicted values and calculations of confidence intervals are shown in Figure 3.4. The trunk girth vs. weight plotted with the confidence curves is shown in Figure 3.5. The coverage probability for this confidence interval (individual) was $92.31 \%$, covering 96 points for a nominal probability of $95 \%$. For $90 \%$ probability the coverage probability, was $82.69 \%$ covering 86 points, with $|\mathrm{CP}-90| \%$ of $7.31 \%$. 
Table 3.3 Regression Analysis and ANOVA for Tree Data [46]

\begin{tabular}{|c|c|c|c|}
\hline \multicolumn{2}{|c|}{ Regression Statistics } & & \\
\hline Multiple R & 0.92 & & \\
\hline R Square & 0.85 & & \\
\hline Adjusted R Square & 0.85 & & \\
\hline Standard Error & 172.18 & & \\
\hline Observations & 80.00 & & \\
\hline \multicolumn{4}{|l|}{ ANOVA } \\
\hline & $d f$ & $S S$ & $M S$ \\
\hline Regression & 1 & 13412097 & 13412097 \\
\hline Residual & 78 & 2312255 & 29644.29 \\
\hline \multirow[t]{2}{*}{ Total } & 79 & 15724351 & \\
\hline & Coefficients & Standard Error & \\
\hline Intercept & -1270.16 & 112.44 & \\
\hline $\mathrm{X}$ Variable 1 & 5.97 & 0.28 & \\
\hline
\end{tabular}

\begin{tabular}{|c|c|c|c|c|c|c|c|c|c|c|c|}
\hline & A & $B$ & C & D & $E$ & $\mathrm{~F}$ & G & $\mathrm{H}$ & 1 & $\mathrm{~J}$ & K \\
\hline 1 & TrunkGirth & Weight & PredictedWeight & Smean & Sind & & & LCImean & UCImean & LCIInd & UCIInd \\
\hline 2 & 406 & 1094 & 1152.25 & 19.50 & 173 & & & 1120 & 1185 & 864 & 1441 \\
\hline 3 & 405 & 1241 & 1146.29 & 19.46 & 173 & & & 1114 & 1179 & 858 & 1435 \\
\hline 4 & 385 & 1017 & 1026.96 & 19.45 & 173 & & & 995 & 1059 & 739 & 1315 \\
\hline 5 & 351 & 726 & 824.09 & 22.85 & 174 & & & 786 & 862 & 535 & 1113 \\
\hline 6 & 465 & 1658 & 1504.28 & 27.51 & 174 & & & 1458 & 1550 & 1214 & 1795 \\
\hline 7 & 450 & 1288 & 1414.78 & 24.68 & 174 & & & 1374 & 1456 & 1125 & 1704 \\
\hline 8 & 375 & 790 & 967.29 & 20.04 & 173 & & & 934 & 1001 & 679 & 1256 \\
\hline 9 & 460 & 1751 & 1474.45 & 26.53 & 174 & & & 1430 & 1519 & 1184 & 1764 \\
\hline 10 & 452 & 1499 & 1426.71 & 25.04 & 174 & & & 1385 & 1468 & 1137 & 1716 \\
\hline 11 & 478 & 1571 & 1581.84 & 30.23 & 175 & Total & 104 & 1532 & 1632 & 1291 & 1873 \\
\hline 12 & 405 & 1023 & 1146.29 & 19.46 & 173 & obs used Training & 80 & 1114 & 1179 & 858 & 1435 \\
\hline 13 & 424 & 1177 & 1259.65 & 20.91 & 173 & mean & 395 & 1225 & 1294 & 971 & 1548 \\
\hline 14 & 205 & 251 & -47.02 & 56.64 & 181 & SSETR & $2,312,255$ & -141 & 47 & -349 & 255 \\
\hline 15 & 425 & 1488 & 1265.62 & 21.02 & 173 & dof & 78 & 1231 & 1301 & 977 & 1554 \\
\hline 16 & 390 & 944 & 1056.79 & 19.30 & 173 & MSETR & 29,644 & 1025 & 1089 & 768 & 1345 \\
\hline 17 & 343 & 733 & 776.36 & 24.14 & 174 & Clevel & 95 & 736 & 817 & 487 & 1066 \\
\hline 18 & 404 & 1084 & 1140.32 & 19.42 & 173 & Tdist & 1.665 & 1108 & 1173 & 852 & 1429 \\
\hline 19 & 395 & 952 & 1086.62 & 19.25 & 173 & RsqTR & 0.853 & 1055 & 1119 & 798 & 1375 \\
\hline 20 & 226 & 278 & 78.28 & 51.14 & 180 & RsqTe & 0.806 & -7 & 163 & -221 & 377 \\
\hline 21 & 299 & 506 & 513.84 & 33.08 & 175 & $\begin{array}{l}\text { Should've } \\
\text { covered }\end{array}$ & 99 & 459 & 569 & 222 & 806 \\
\hline 22 & 463 & 1838 & 1492.35 & 27.12 & 174 & covered [indiv] & 96 & 1447 & 1537 & 1202 & 1782 \\
\hline 23 & 334 & 610 & 722.66 & 25.74 & 174 & CP [indiv] & $92.31 \%$ & 680 & 766 & 433 & 1012 \\
\hline 24 & 438 & 1197 & 1343.18 & 22.73 & 174 & & & 1305 & 1381 & 1054 & 1632 \\
\hline 25 & 410 & 1035 & 1176.12 & 19.71 & 173 & & & 1143 & 1209 & 888 & 1465 \\
\hline
\end{tabular}

Figure 3.4 Calculations of Confidence Intervals for Linear Regression in a Spreadsheet 


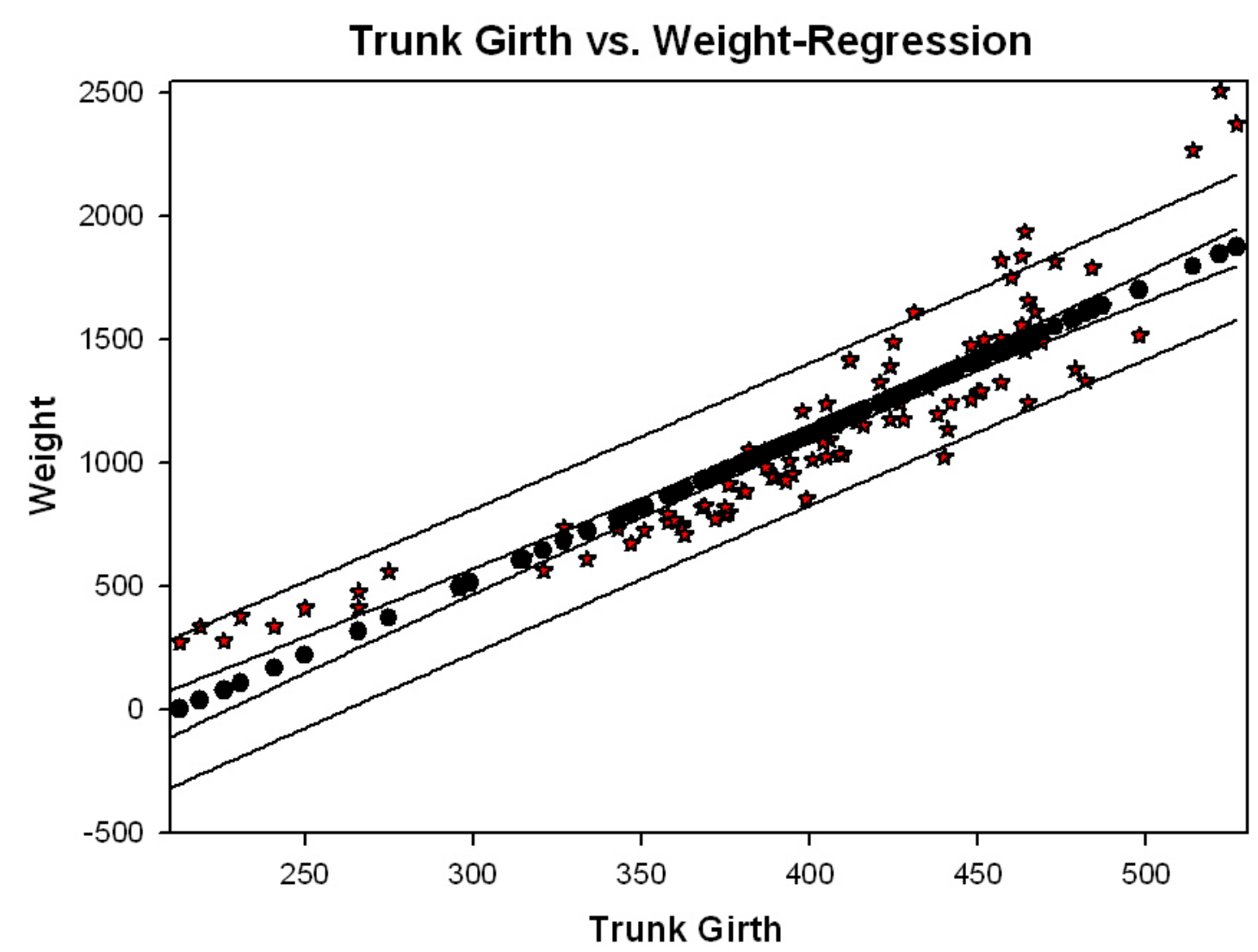

Figure 3.5 Trunk Girth vs. Weight for the Tree Data [46] using Regression Predicted Weight Values Given By Dots, Actual Weight Values Given By Stars, and Solid Lines 95\% Confidence Curves

\subsection{Development of Confidence Interval via Non-linear Regression}

Constructing confidence interval via non-linear regression is a straightforward process except for obtaining values for the elements in the Jacobian matrix, $\mathrm{F}$ (Equation 16) and $\mathrm{f}_{0}{ }^{T}$ matrices. The $\mathrm{F}$ and $\mathrm{f}_{0}{ }^{T}$ contain the derivatives of the output with respect to the parameters used to estimate $y$. This calculation of the elements of $\mathrm{F}$ and $\mathrm{f}_{0}{ }^{T}$ matrices was adopted from [16]. Two terms defined by the authors in [16] are net ${ }_{j}^{[\beta]}$ and layer ${ }^{[\beta]}$ given in Equation (49) and (50) respectively. The net ${ }_{j}^{[\beta]}$ is summation of the outputs from the nodes of layer $\beta-1$ entering node $j$ in layer $\beta$. The layer ${ }^{[\beta]}$ is the response of layer $\beta$ for a given set of inputs, which is the summation of $\operatorname{net}_{j}^{[\beta]}$ functions for layer $\beta$.

$$
\operatorname{net}_{j}^{[\beta]}=\left(\sum_{i=1}^{n} w_{i}^{[\beta-1]} \operatorname{zout}_{i}^{[\beta-1]}\right)-b_{j}
$$


where,

$n=$ Number of nodes in layer $\beta-1$, and

$b_{j}=$ Bias term of node $\mathrm{j}$

$$
\operatorname{layer}^{[\beta]}=\sum_{i=1}^{m} \operatorname{net}_{j}^{[\beta]}
$$

where,

$\mathrm{m}=$ Number of nodes in layer $\beta$

Equation (51) expresses the derivative of the output $y$ with respect to the weights and the biases, $\frac{\partial y}{\partial w_{\gamma}^{[\alpha]}}$, where $\gamma$ is the specific weight value of layer $\alpha$. The terms used in Equation (51), $\frac{\partial y}{\partial \text { net }^{[m]}}, \frac{\partial \text { net }^{[\mathrm{m}]}}{\partial \operatorname{layer}^{[m-1]}}, \frac{\partial \text { layer }^{[\mathrm{m}-1]}}{\partial \operatorname{layer}^{[m-2]}}, \frac{\partial \text { layer }^{[\alpha+2]}}{\partial \text { net }_{\gamma}^{[\alpha+1]}}, \frac{\partial \text { net }^{[\alpha+1]}}{\partial w_{\gamma}^{[\alpha]}}$ are given in Equation (52), (53), (54),(55)

and (56) respectively.

$$
\begin{gathered}
\frac{\partial y}{\partial w_{\gamma}^{[\alpha]}}=\frac{\partial y}{\partial \text { net }^{[m]}} \frac{\partial \text { net }^{[\mathrm{m}]}}{\partial \text { layer }^{[m-1]}} \frac{\text { layer }^{[\mathrm{m}-1]}}{\partial \text { layer }^{[m-2]}} \cdots \frac{\partial \text { layer }^{[\alpha+3]}}{\partial \text { layer }^{[\alpha+2]}} \frac{\partial \text { layer }^{[\alpha+2]}}{\partial \text { net }_{\gamma}^{[\alpha+1]}} \frac{\partial \mathrm{net}^{[\alpha+1]}}{\partial w_{\gamma}^{[\alpha]}} \\
\frac{\partial y}{\partial \text { net }^{[m]}}= \begin{cases}1 & \text { if the output neuron has linear activation function, } \\
y(1-y) & \text { if the output neuron has sigmoidal activation function. }\end{cases} \\
\frac{\partial \text { net }^{[\mathrm{m}]}}{\partial \text { layer }^{[m-1]}}=\sum_{i=1}^{n} \frac{\partial \text { net }^{[\mathrm{m}]}}{\partial \text { net }_{i}^{[m-1]}} \\
\frac{\partial \text { layer }^{[\mathrm{m}-1]}}{\partial \text { layer }^{[m-2]}}=\sum_{j=1}^{p}\left(\sum_{i=1}^{n} \frac{\partial \text { net }_{j}^{[m-1]}}{\partial \text { net }_{i}^{[m-2]}}\right)
\end{gathered}
$$

Where,

$p=$ Number of nodes in layer $m-1$.

$$
\begin{gathered}
\frac{\partial_{\text {layer }}^{[\alpha+2]}}{\partial \operatorname{net}_{\gamma}^{[\alpha+1]}}=\sum_{i=1}^{p} \frac{\partial \operatorname{net}_{j}^{[\alpha+2]}}{\partial \operatorname{net}_{\gamma}^{[\alpha+1]}} \\
\frac{\partial \operatorname{net}^{[\alpha+1]}}{\partial w_{\gamma}^{[\alpha]}}=\operatorname{zout}_{\gamma}^{[\alpha]}
\end{gathered}
$$


A general form of $\frac{\partial \text { net }_{\lambda}^{[\phi+1]}}{\partial \operatorname{net}_{\zeta}^{[\phi]}}$ is given in Equation(57), where $\phi$ is an arbitrary layer, $\lambda$ is an arbitrary node in layer $\phi+1, \zeta$ is an arbitrary node in layer $\phi$, and $A$ is the number of nodes in layer $\phi$.

$$
\frac{\partial \mathrm{net}_{\lambda}^{[\phi+1]}}{\partial \operatorname{net}_{\zeta}^{[\phi]}}=\sum_{a=1}^{A}\left\{w_{a}^{[\phi]} \operatorname{zout}_{a}^{[\phi]}\left(1-\operatorname{zout}_{a}^{[\phi]}\right)\right\}
$$

\subsubsection{Steps for Finding Confidence Intervals Using Non-Linear Regression}

The steps to find confidence interval using non-linear regression are given as follows:

1. Split the data into training and test data sets.

2. Scale the data.

3. Train the network using the training data set.

4. Calculate $s^{2}$ using Equation (19) for the training data. (Page 19)

5. Predict the test data.

6. Find first order partial derivatives of output with respect to the parameters (weights), i.e., matrices $\mathrm{F}$ and $\mathrm{f}_{0}$ using Equations (49) to(57). (Pages 33-34-Code given in Appendix C)

7. Calculate the SVD of F and diagonal elements of the $H$ matrix as given on pages 19 and 20 .

8. Select appropriate confidence level and respective t-distribution value.

9. Calculate the correction factor $\lambda$ given by Equation (30).

10. Calculate confidence intervals for the training and test data using Equation (31). (Page 21)

\subsubsection{Validation of the NLR model}

This model was developed on the information presented by Chryssolouris et al [16]. However, calculating the matrix is a complex process and calculating its inverse is often impossible if the matrix becomes singular. Therefore, the method described in Rivals and Personnaz [39, 40] was used. The data presented in Chryssolouris et.al. [16] could not be found after a thorough search and hence this model was not able to be validated.

The major problem with the technique presented in Chryssolouris et.al. [16] is the values in the $\mathrm{F}^{T}$.F matrix. Sometimes the values are so small that the matrix becomes singular and the inverse of the matrix cannot be calculated. Therefore, the confidence interval cannot be 
predicted or instability occurs, which can make the confidence intervals several orders of magnitude too wide in extreme cases [13]. As noted in R.D. De Veaux et.al .[13], non-linear regression methods are prone to cause several problems when applying to small samples. The ratio of the number of weights vs. training data is very important.

One more problem with the NLR technique is the number of degrees of freedom, which is the (number of observations - number of parameters). The number of parameters, total number of weights and biases, can exceed the number of observations, and that is not appropriate for these calculations. However, the advantage of this method is that it gives results quickly, and it does not require higher computational powers.

\subsection{Development of Confidence Interval via Bootstrap Estimation}

The development of a confidence interval using bootstrap estimation is a straightforward process. The main problem with this method is that it is computationally expensive. The steps involved in this technique were given in Section 2.1.2. The bootstrap estimation algorithm requires that the model be retrained $B$ times, which is the number of bootstrap samples. Typically $B$ is in the range $20 \leq B \leq 200$ [24]. This method doesn't require matrix inversion or the existence of derivatives, and it can be shown that [24] the bootstrap pairs sampling estimate (as $B \rightarrow \infty$ ) agrees with the standard least squares formula $\left[\mathrm{x}_{i}^{T}\left(X^{T} X\right)^{-1} \mathrm{x} i \hat{\sigma}^{2}\right]^{1 / 2}, X$ denoting the design matrix having rows $\mathrm{x}_{i}$.

\subsubsection{Validation of Bootstrapping Model}

For validation purposes, the method given in R. Tibshirani [24] was used. This method requires calculating the standard deviation estimators for 25 simulations. In this example, four input Gaussian variables with mean zero and variance one were used. This predictor set was generated once and then fixed for all 25 simulations. The value $y$ was generated as given in Equation (58). There were 100 observations in each training set. The architecture used for training was 4-2-1.

$$
y=3 f\left(2 x_{1}+4 x_{2}+3 x_{3}+3 x_{4}\right)+3 f\left(2 x_{1}+4 x_{2}-3 x_{3}+3 x_{4}\right)+\varepsilon
$$

where $f()$ is sigmoidal function given as $f(x)=\frac{1}{1+\exp (-x)}$ and $\varepsilon$ is Gaussian with a mean of zero and standard deviation of 0.7 . 
Table 3.4 Median Standard Error and Standard Deviation for 25 Simulations.

\begin{tabular}{|c|c|c|c|}
\hline $\begin{array}{c}\text { Simulation } \\
\text { Number }\end{array}$ & $\begin{array}{c}\text { Median of } \\
\text { Standard error }\end{array}$ & $\begin{array}{c}\text { Standard Deviation of } \\
\text { Predicted }\end{array}$ & $\begin{array}{c}\text { MSE } \\
\text { Training }\end{array}$ \\
\hline 1 & 1.69 & 1.67 & 1.30 \\
\hline 2 & 1.66 & 1.67 & 1.32 \\
\hline 3 & 1.76 & 1.68 & 1.29 \\
\hline 4 & 1.73 & 1.68 & 1.37 \\
\hline 5 & 1.81 & 1.57 & 1.50 \\
\hline 6 & 1.65 & 1.66 & 1.43 \\
\hline 7 & 1.82 & 1.42 & 1.93 \\
\hline 8 & 1.70 & 1.54 & 1.61 \\
\hline 9 & 1.70 & 1.45 & 1.89 \\
\hline 10 & 1.65 & 1.66 & 1.35 \\
\hline 11 & 1.73 & 1.67 & 1.34 \\
\hline 12 & 1.72 & 1.66 & 1.30 \\
\hline 13 & 1.74 & 1.68 & 1.29 \\
\hline 14 & 1.65 & 1.66 & 1.30 \\
\hline 15 & 1.74 & 1.65 & 1.38 \\
\hline 16 & 1.64 & 1.53 & 1.71 \\
\hline 17 & 1.74 & 1.39 & 1.96 \\
\hline 18 & 1.69 & 1.58 & 1.51 \\
\hline 19 & 1.83 & 1.59 & 1.51 \\
\hline 20 & 1.71 & 1.47 & 1.79 \\
\hline 21 & 1.80 & 1.65 & 1.32 \\
\hline 22 & 1.74 & 1.56 & 1.59 \\
\hline 23 & 1.75 & 1.59 & 1.50 \\
\hline 24 & 1.71 & 1.66 & 1.30 \\
\hline 25 & 1.75 & 1.55 & 1.51 \\
\hline Median & 1.73 & 1.65 & \\
\hline
\end{tabular}

Let $\hat{s}_{i k}$ be the estimated standard deviation of $f(\mathrm{x} ; \hat{\theta})$ for the $k^{\text {th }}$ simulated sample. The median over the training cases of the estimated standard deviation of $\hat{y}_{i}$ for the $k^{\text {th }}$ simulated sample is given by $s e_{k} \equiv \operatorname{median}_{i}\left(\hat{s}_{i k}\right)$. Let $s_{i}$ be the actual standard deviation of $f(\mathrm{x} ; \hat{\theta})$. The 
median of the actual values of the standard deviation was found to be 1.65 and $s e_{k}$ calculated over 25 simulated samples was 1.73, which is very close to the actual standard deviation. For the example given by R. Tibshirani [24], the actual standard deviation was found to be 0.86 , and for the bootstrap sampling $\left(s e_{k}\right)$, it was found to be 0.96 . Since the values of standard deviation were close, the developed model was treated as a valid model. The median of the standard errors and actual standard deviation values are given in Table 3.4.

\subsection{Development of Confidence Interval via Maximum-likelihood Estimation (MLE)}

As mentioned by Papadopoulos et. al. [23] there are two sources of uncertainty - noise variance $\sigma_{v}^{2}$ and uncertainty variance $\sigma_{m}^{2}$. The data noise is modeled as additive Gaussian independently, identically distributed (i.i.d.) errors with zero mean and variance $\sigma_{v}^{2}$. The independently, identically distributed are those variables that have the same probability distribution as any of the others and are mutually independent. The later uncertainty variance, $\sigma_{m}^{2}$, is referred to as the model uncertainty variance. In case of the ANNs, the model certainty is due to weight misspecification.

As further explained by Papadopoulos et.al.[23], these two sources of uncertainties are assumed to be independent, and the total prediction variance is calculated as $\sigma_{\text {total }}^{2}=\sigma_{v}^{2}+\sigma_{m}^{2}$. Under maximum-likelihood training, a separate network is used to obtain the input independent

estimate of $\sigma_{m}^{2}$. The data noise variance $\sigma_{v}^{2}$ is calculated using the standard formula to calculate the variance in the data. The steps to calculate the confidence interval are given as follows:

1. Split the data into training and test data.

2. Scale the data.

3. Train the network.

4. Calculate $\sigma_{v}^{2}$ for the data used to predict the output.

5. Scale the values back.

6. Calculate the errors $\left(\mathrm{y}_{\text {out }}-\right.$ target$)$ for each data set.

7. Scale the data again. 
8. Train a separate network using the same inputs used in step three with target values as $\left(\mathrm{y}_{\text {out }}-\text { target }\right)^{2}$.

9. Approximating the target values of the network from step six as $\sigma_{m}^{2}$, the total noise variance $\sigma_{\text {total }}^{2}$ can be calculated.

10. Scale the data back.

11. Select an appropriate confidence level and respective student t-distribution value.

12. Use $y_{-}$out $\mathrm{t}_{\mathrm{k}}-\mathrm{t} \sigma_{\text {total }}^{2} \leq \mathrm{f}(\mathrm{x}) \leq y_{-}$out $\mathrm{k}+\mathrm{t} \sigma_{\text {total }}^{2}$ to find confidence intervals for the training data [31].

13. Repeat steps two to 12 for the test data.

\subsubsection{Validation of the MLE model}

The MLE model was developed based on the information mentioned by J. G. Carney, P. Cunningham, and U. Bhagwan [31] and R. Dybowski [32]. Carney et.al.[31] provided important insight over the information for predicting confidence intervals using MLE. The authors test the models using Equation (60). However, Carney et al. [31] did not have sufficient information to validate this model. The authors provided information only on the efficiency of the model. Dybowski [32] provided the basic information needed to construct the confidence intervals, but did not have information on the validation of the model nor does the give any example for comparison.

\subsection{Comparison of Confidence Intervals for Linear Regression and Neural Networks}

Computations of confidence intervals for linear regression were discussed in Section 3.2. Tree data [47] were used to compute the confidence intervals, and to recall the coverage probability for individual observations was $92.31 \%$ for linear regression with $95 \%$ nominal probability and $82.69 \% \mathrm{CP}$ with $90 \%$ nominal probability. The same data were used to compute confidence intervals using the above discussed techniques. The neural network and confidence interval parameters are given in Table 3.5. 
Table 3.5 Neural Network and Confidence Interval Parameters for 95\% Nominal Probability

\begin{tabular}{|l|c|}
\hline Number of Observations For Training & 80 \\
\hline Number of Observations For Testing & 24 \\
\hline Number of Hidden Neurons & 2 \\
\hline Number of Iterations & 700 \\
\hline Total Number of Parameters & 73 \\
\hline Degrees of Freedom & 1.99 \\
\hline t-Distribution Value & Sigmoid \\
\hline Activation Function Between Input and Hidden & Linear \\
\hline Activation Function Between Hidden and Output & 7 \\
\hline
\end{tabular}

The details of the results obtained from neural network and the confidence estimating techniques is listed in Table 3.6 and Table 3.7. According to the CP-95 values, linear regression has the least difference from the nominal probability. Confidence curves obtained via all the techniques and by linear regression are superimposed on one graph, shown in Figure 3.9. Confidence curves obtained via non-linear regression (NLR) with (NLR-wCF) and without the correction factor for neural networks are shown in Figure 3.6. Although linear regression had the best CP-95 value among all the techniques, bootstrapping was the best CP-90 value.

Table 3.6 Results Obtained by Neural Network and Confidence Estimation Techniques with 95\% Probability

\begin{tabular}{|c|c|c|c|c|c|}
\hline Total Observations & 104 & \multicolumn{2}{|c|}{ Observations for Training } & 80 & \\
\hline Training MSE & $1,416,605$ & \multicolumn{2}{|c|}{ Test MSE } & 883,801 & \\
\hline Training R-sq & 0.91 & \multicolumn{2}{|c|}{ Test R-sq } & 0.85 & \\
\hline \multirow[t]{2}{*}{ Nominal (\%) } & 95 & \multicolumn{2}{|c|}{ Points should've covered } & 99 & \\
\hline & NLR & NLR-wCF & Boot & MLE & LR \\
\hline Points Covered by CIs & 89 & 104 & 102 & 88 & 96 \\
\hline Points Not covered by CIs & 15 & 0 & 2 & 16 & 8 \\
\hline CP (\%) & $85.58 \%$ & $100.00 \%$ & $98.08 \%$ & $84.62 \%$ & $92.31 \%$ \\
\hline CP - Nominal (\%) & $9.42 \%$ & $-5.00 \%$ & $-3.08 \%$ & $10.38 \%$ & $2.69 \%$ \\
\hline
\end{tabular}


Table 3.7 Results Obtained by Neural Network and Confidence Estimation Techniques with 90\% Probability

\begin{tabular}{|c|c|c|c|c|c|}
\hline Total Observations & 104 & \multicolumn{2}{|c|}{ Observations for Training } & 80 & \\
\hline Training MSE & $1,416,605$ & \multicolumn{2}{|c|}{ Test MSE } & 883,801 & \\
\hline Training R-sq & 0.91 & \multicolumn{2}{|c|}{ Test R-sq } & 0.85 & \\
\hline \multirow[t]{2}{*}{ Nominal (\%) } & 90 & \multicolumn{2}{|c|}{ Points should've covered } & 94 & \\
\hline & NLR & NLR-wCF & Boot & MLE & LR \\
\hline Points Covered by CIs & 85 & 104 & 93 & 85 & 86 \\
\hline Points Not covered by CIs & 19 & 0 & 11 & 19 & 18 \\
\hline CP (\%) & $81.73 \%$ & $100.00 \%$ & $89.42 \%$ & $81.73 \%$ & $82.69 \%$ \\
\hline CP - Nominal (\%) & $8.27 \%$ & $-10.00 \%$ & $0.58 \%$ & $8.27 \%$ & $-7.31 \%$ \\
\hline
\end{tabular}

\section{Trunk Girth vs Weight}

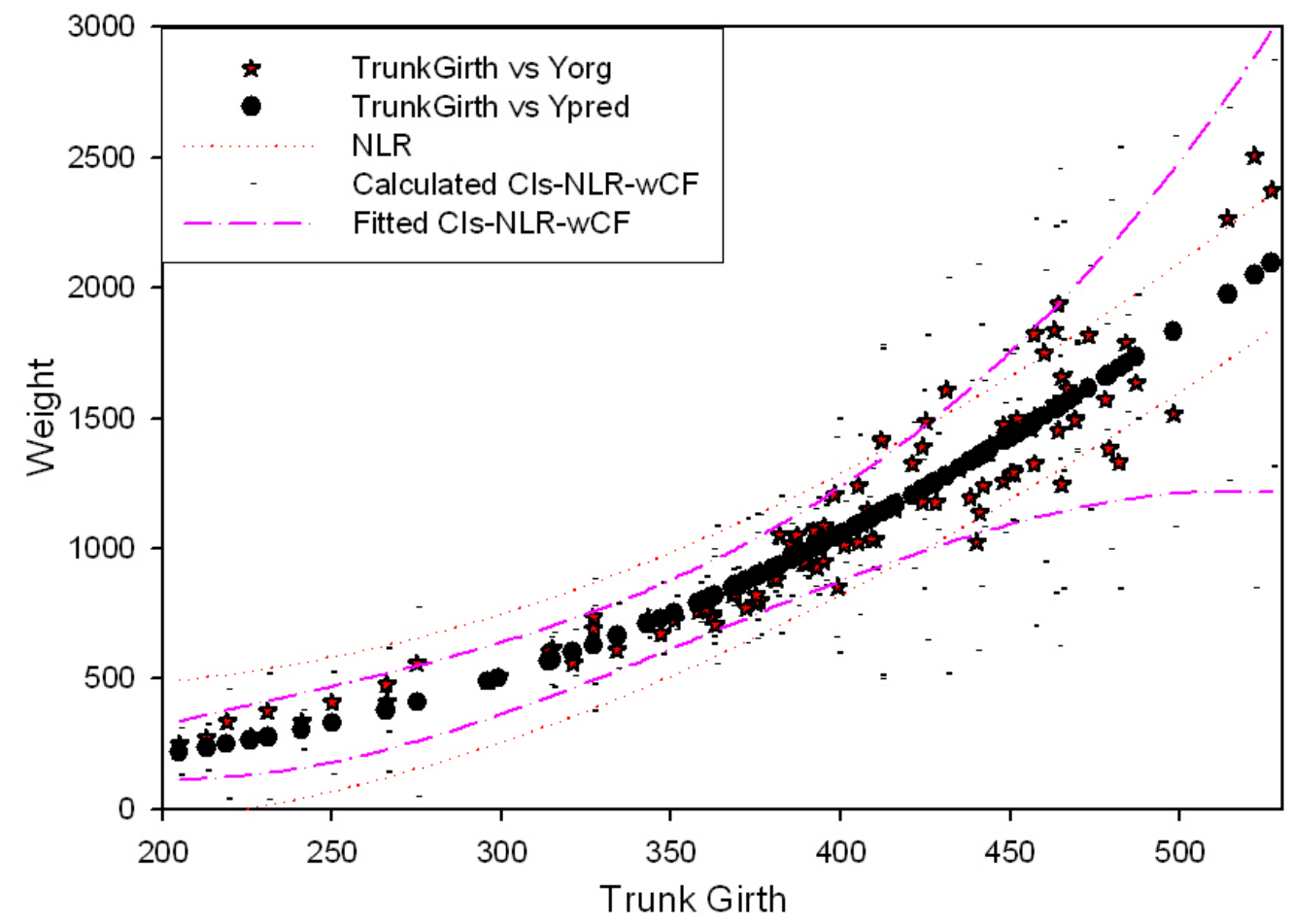

Figure 3.6 Trunk Girth vs. Weight Using Neural Networks with Confidence Curves via Non-Linear Regression-with and without the Correction Factor (CF) with Confidence Level 95\%

Both fitted line and actual data points for NLR with the CF are shown in Figure 3.6. Confidence intervals obtained without the $\mathrm{CF}$ are wide for initial trunk girth values, then narrow around the high data density area. Confidence intervals obtained using the CF cover all the points, hence, obtaining coverage probability of $100 \%$. Figure 3.7 shows the confidence curves 
obtained using the bootstrap estimation technique. Bootstrap spreads out when the data density is low and narrow when data density is high.

Confidence curves obtained using the maximum likelihood estimation (MLE) technique are shown in Figure 3.8. Results obtained by MLE were quite close to results obtained by NLR without the CF with CP of $84.62 \%$. MLE failed to obtain spread like NLR and bootstrapping but it obtained CP similar to NLR. The spread was constant similar to the spread obtained by linear regression.

It should be noted that all these techniques assume that the predicted output is very close to the actual values, which was not a case in this example. All the curves are superimposed in one graph and shown in Figure 3.9. The spread obtained by bootstrapping was very close to the expectation. Linear regression and MLE obtained a constant spread for all data points. For 90\% probability, linear regression obtained $|\mathrm{CP}-90|$ of $7.31 \%$, which was close to the CP-90 obtained by NLR and MLE.

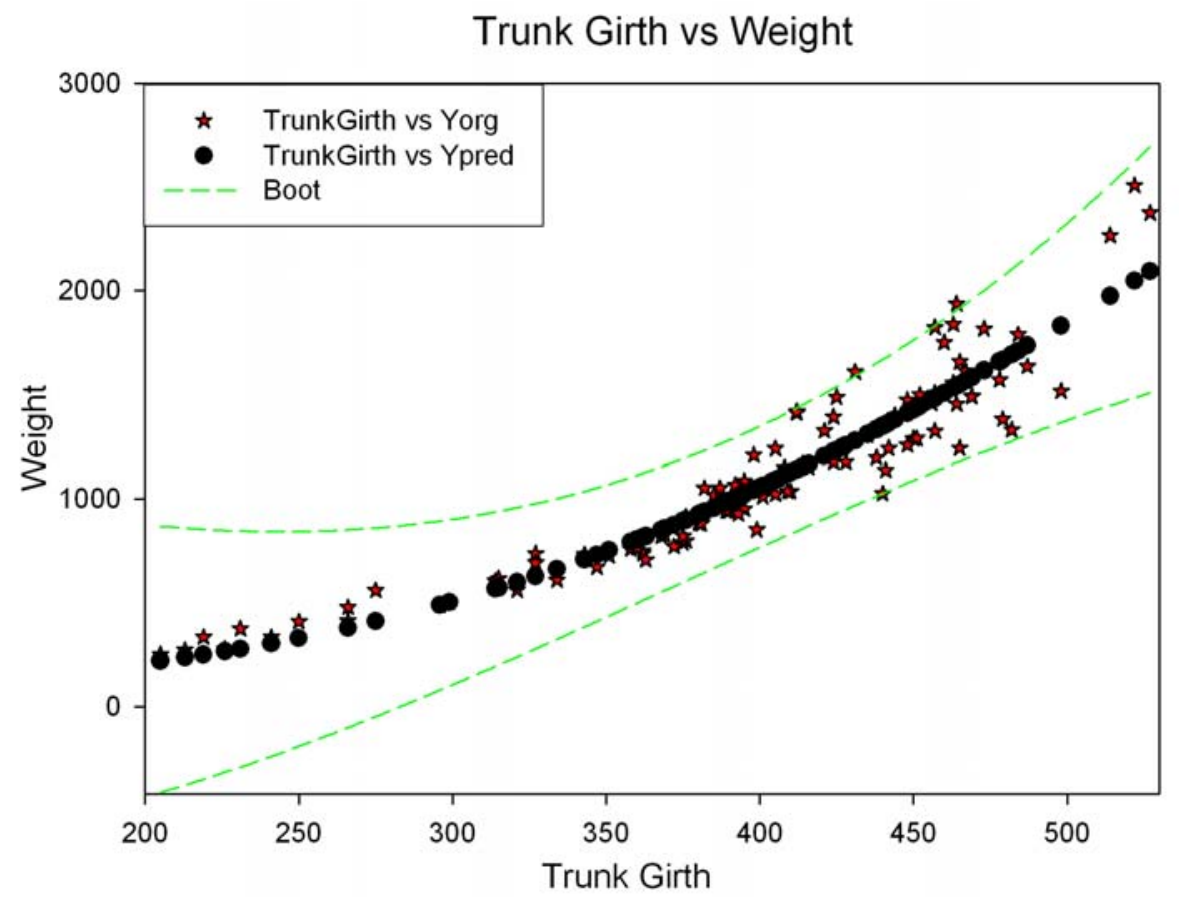

Figure 3.7 Trunk Girth vs. Weight Using Neural Networks with Confidence Curves Using Bootstrap with Confidence Level 95\% 


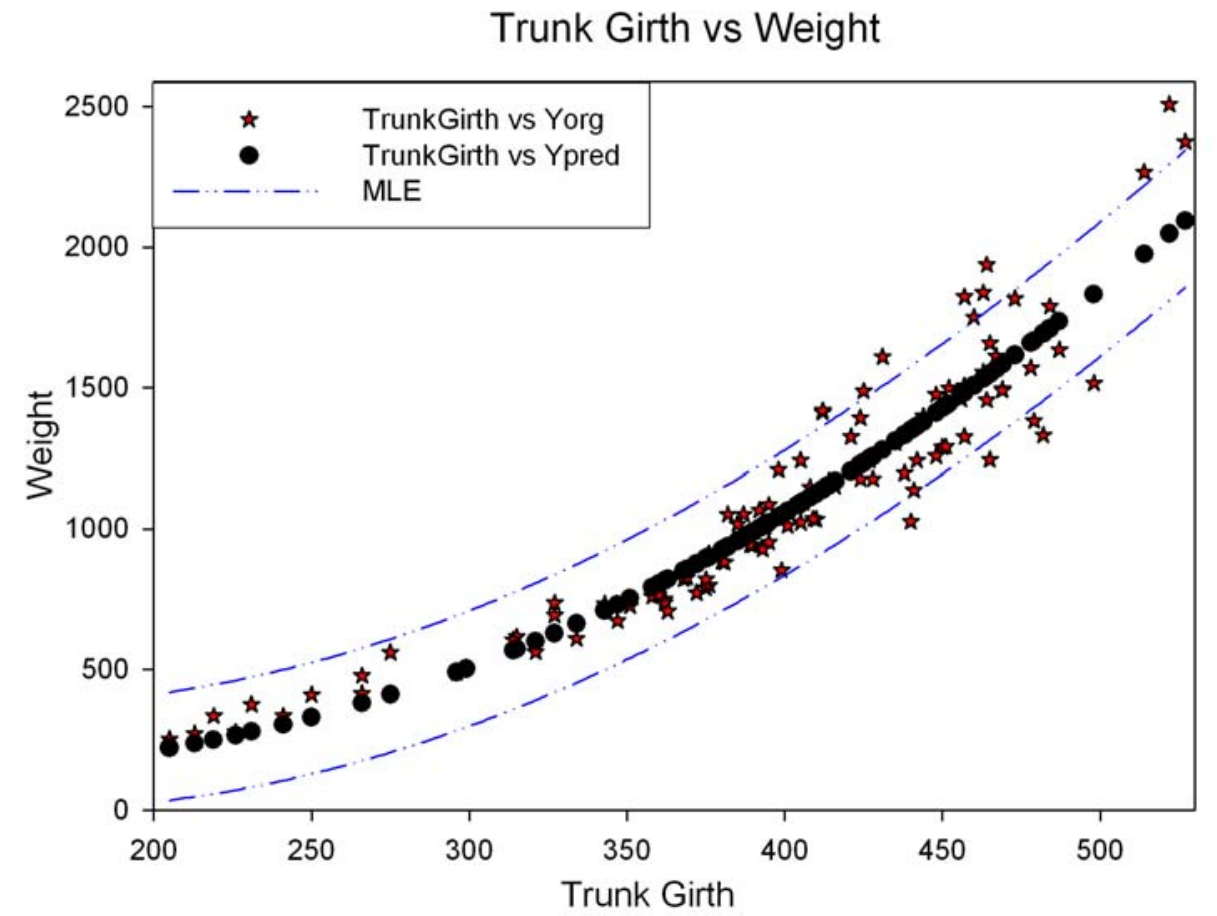

Figure 3.8 Trunk Girth vs. Weight Using Neural Networks with Confidence Curves Using MLE with Confidence Level 95\% 


\section{Trunk Girth vs Weight}

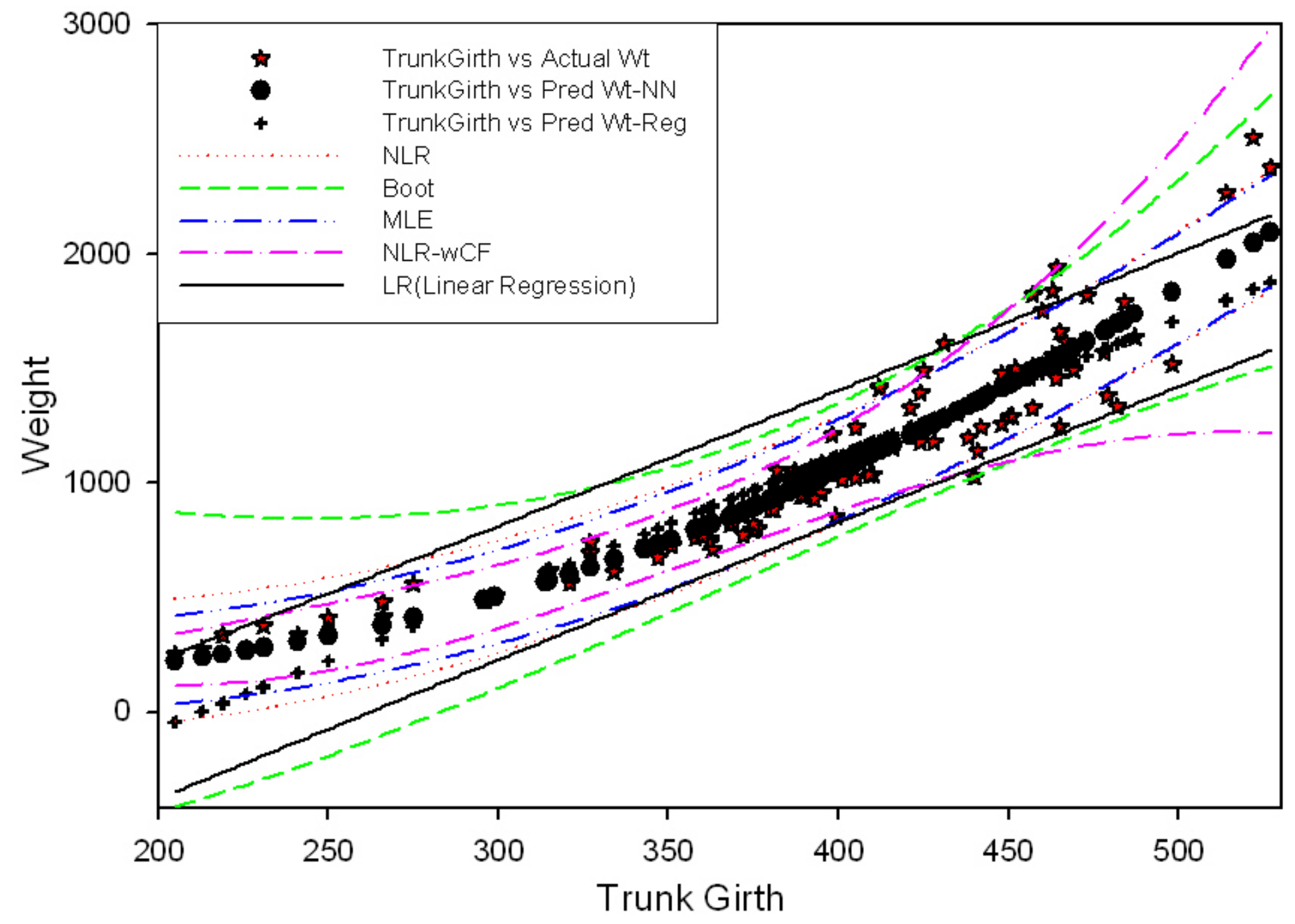

Figure 3.9 Confidence Curves Obtained via All Techniques 


\section{Assessing Confidence Estimation Techniques}

\subsection{Introduction}

The assessment of the confidence estimation techniques was done by following the methods suggested by R.D. De Veaux et.al.[13], G. Papadopoulos et.al.[23], J. R. Donaldson et. al.[33], and J. T. G. Hwang et.al.[34]. The estimation techniques were the non-linear regression, bootstrapping, and maximum likelihood estimation. The coverage probability (CP) of the confidence intervals was computed to assess the confidence estimation techniques. $\mathrm{CP}$ is evaluated by calculating the percentage of target (original y) data points that lie within the confidence interval range on the predicted data. Therefore, $\mathrm{CP}$ is the probability that the true value lies within the confidence interval [23]. The performance of a confidence measure can be assessed in practice by estimating the observed $\mathrm{CP}$ and comparing it with the nominal value. Also, a confidence estimation technique will be good if the $\mathrm{CP}$ value is close to the nominal value.

It should be noted that for the maximum likelihood estimation technique, the results could vary depending on the number of epochs used for training the residuals as target values. The literature does not provide any information on how long the residuals are trained. Moreover, the number of hidden layer neurons can also be changed for the residual training, and the literature again fails to provide the information.

Various data sets from the literature were used to assess the estimation techniques. Fifteen simulations were taken for each estimation technique and then mean CP was calculated. All the techniques assume that the error or noise in the data or in the weights is Gaussian distributed, except NLR, which uses the correction factor as explained in Section 2.1.1. If the correction factor is used, it results in coverage of all the actual points, hence, obtaining a CP of $100 \%$. However, the comparing criterion of this research was based on coverage probabilities, therefore, the NLR estimation technique without the correction factor was used.

Four different cases were studied for the assessment of these confidence estimation techniques. For Case A, a synthetic data set was created using a polynomial equation with five input variables, one output variable, and it had 250 data points. Case B was taken from McKim [46], which had two input and one output variables. The input variables were flow of pumps in gpm and head in inches and the output variable was the actual cost of the pump. This data set had 23 observations. 
Synthetic data presented in J. G. Carney [31], which had 1000 observations for training and 1000 observations for testing with one input and output variable, was for Case C. Tree data [46] used for Case D was the same data set previously used to compare the techniques. This data set had trunk girth of the tree as an input variable, weight of the tree as an output variable, and had 104 observations.

\subsection{Case Studies}

\subsubsection{Case A}

A data set with 250 rows with five input variables using Equation (59) was generated. This data set was without any noise, and it was easy to train. Out of 250 data points, 175 were used for training and 75 were used for testing. The data set used is given in Table A-1 of Appendix A. The values of $\mathrm{X}$ were generated randomly, ranging from zero to one. The neural net architecture was 5-2-1. The learning rate was kept constant, as 0.2 and 100 epochs were sufficient for a good fit for the training and test data. Usually preferred, sigmoid activation function for the hidden layer and linear activation function for the output layer were selected. The details of training and confidence interval calculation parameters are given in Table 4.1. The number of bootstrap samples used was 20, which is the lower limit of samples recommended [24] but good enough for complicated problems such as neural networks.

$$
y=2 x_{1}^{2}+3 x_{2}^{3}+4 x_{3}+x_{4}^{1 / 2}+4 x_{5}
$$

Table 4.1 Neural Network and Confidence Interval Parameters for Case A

\begin{tabular}{|l|c|}
\hline Number of Observations For Training & 175 \\
\hline Number of Observations For Testing & 75 \\
\hline Number of Inputs & 5 \\
\hline Number of Hidden Neurons & 2 \\
\hline Learning Rate & 0.2 \\
\hline Number of Iterations & 100 \\
\hline Total Number of Parameters & 15 \\
\hline Degrees of Freedom & 160 \\
\hline Activation Function Between Input And Hidden & Sigmoid \\
\hline Activation Function Between Hidden And Output & Linear \\
\hline
\end{tabular}


For Case A at the 95\% confidence interval, NLR obtained the best average $|\mathrm{CP}-95|$ of $0.76 \%$. Bootstrap estimation obtained an average $|\mathrm{CP}-95|$ of $3.29 \%$ over 15 simulations, whereas MLE obtained, average $|\mathrm{CP}-95|$ of $3.45 \%$ with the coverage of 232 points inside the confidence intervals. Table 4.2 gives the summary for this case study. These programs were run on a computer with a Pentium 4, $2.20 \mathrm{GHz}$ processor and $640 \mathrm{MB}$ RAM. Results for all the 15 simulations for nominal probability of $95 \%$ are given in Table C-1 of Appendix C. Results for $90 \%$ nominal probability are summarized in Table 4.3 . When the confidence level or nominal probability was $90 \%$, bootstrap was found to be the best of the three techniques with the average CP-90 of $6.91 \%$. Results for this confidence level are given in Table C-2. Standard deviation (SD) of all the CPs obtained over 15 simulations was also computed and given in Table 4.2 and Table 4.3. It can be seen that bootstrapping had the highest SD over 15 simulations and NLR had the lowest SD.

Table 4.2 Results for Case A: Average Taken Over 15 Simulations for $95 \%$ Confidence Level

\begin{tabular}{|c|l|c|c|c|}
\hline & & Non-linear Regression & Bootstrapping & MLE \\
\hline 1 & Total Points Used & 250 & 250 & 250 \\
\hline 2 & Nominal & $95 \%$ & $95 \%$ & $95 \%$ \\
\hline 3 & Expected Coverage & 238 & 238 & 238 \\
\hline 4 & Covered Points & 239 & 241 & 232 \\
\hline 5 & Average CP $(\%)$ & $95.63 \%$ & $96.37 \%$ & $92.88 \%$ \\
\hline 6 & Average $\mid \mathrm{CP}-95(\%)$ & $0.76 \%$ & $3.29 \%$ & $3.45 \%$ \\
\hline 7 & Range & $94.00-96.80$ & $90.00-100.00$ & $89.20-100.00$ \\
\hline 8 & SD of CPs & 0.61 & 3.57 & 3.14 \\
\hline
\end{tabular}

Table 4.3 Results for Case A: Average Taken Over 15 Simulations for 90\% Confidence Level

\begin{tabular}{|c|l|c|c|c|}
\hline & & Non-linear Regression & Bootstrapping & MLE \\
\hline 1 & Total Points Used & 250 & 250 & 250 \\
\hline 2 & Nominal & $90 \%$ & $90 \%$ & $90 \%$ \\
\hline 3 & Expected Coverage & 225 & 225 & 225 \\
\hline 4 & Covered Points & 205 & 220 & 200 \\
\hline 5 & Average CP $(\%)$ & $82.13 \%$ & $88.16 \%$ & $80.16 \%$ \\
\hline 6 & Average $\mid \mathrm{CP}-90(\%)$ & $7.87 \%$ & $6.91 \%$ & $12.51 \%$ \\
\hline 7 & Range & $80.80-86.80$ & $73.20-99.20$ & $74.40-100.00$ \\
\hline 8 & SD of CPs & 1.43 & 8.48 & 8.27 \\
\hline
\end{tabular}




\subsubsection{Case B}

For case B, cost data were taken from McKim [46], which are also given in Table 2-1. These data contained two input variables and one output variable with 23 data rows. Out of 23 data rows, the first 19 were used for training and the remaining four for testing. The number of hidden layer neurons was two with 10 degrees of freedom, which were used in calculations of non-linear regression. The net architecture was 2-2-1, and no excess noise was added. Detailed training and confidence interval parameters are given in Table 4.4. Summary of the results obtained for Case B are given in Table 4.5.

Table 4.4 Neural Network and Confidence Interval Parameters for Case B

\begin{tabular}{|l|c|}
\hline Number of Observations For Training & 19 \\
\hline Number of Observations For Testing & 4 \\
\hline Number of Inputs & 2 \\
\hline Number of Hidden Neurons & 0.2 \\
\hline Learning Rate & 200 \\
\hline Number of Iterations & 9 \\
\hline Total Number of Parameters & 10 \\
\hline Degrees of Freedom & Sigmoid \\
\hline Activation Function Between Input and Hidden & Linear \\
\hline Activation Function Between Hidden and Output & \\
\hline
\end{tabular}

Table 4.5 Results for Case B: Average Taken Over 15 Samples for 95\% Confidence Level

\begin{tabular}{|c|l|c|c|c|}
\hline & & Non-linear Regression & Bootstrap & MLE \\
\hline 1 & Total Points Used & 23 & 23 & 23 \\
\hline 2 & Nominal & $95 \%$ & $95 \%$ & $95 \%$ \\
\hline 3 & Expected Coverage & 22 & 22 & 22 \\
\hline 4 & Covered Points & 22 & 22 & 21 \\
\hline 5 & Average CP (\%) & $95.65 \%$ & $94.20 \%$ & $90.72 \%$ \\
\hline 6 & Average $\mid \mathrm{CP}-95(\%)$ & $0.65 \%$ & $1.67 \%$ & $4.62 \%$ \\
\hline 7 & Range & $95.65-95.65$ & $91.30-95.65$ & $82.61-95.65$ \\
\hline 8 & SD of CPs & 0.00 & 2.12 & 3.98 \\
\hline
\end{tabular}


For Case B, bootstrapping and NLR obtained average |CP-95| of $0.65 \%$ and $1.67 \%$ respectively, whereas MLE had average $|\mathrm{CP}-95|$ of $4.62 \%$. Figure 4.1 show the obtained confidence intervals by all the techniques for the nominal probability of $95 \%$. The results for all simulations are given in Table C-3 of Appendix C. Results obtained for Case B with nominal probability of $90 \%$ are given in Table 4.6. For $90 \%$ nominal probability, NLE and bootstrap obtained average $|\mathrm{CP}-95|$ of $2.17 \%$ and $2.35 \%$, respectively. MLE obtained the worst average |CP-95| of $8.55 \%$. The results for all simulations are given in Table C-4.

Table 4.6 Results for Case B: Average Taken Over 15 Samples for $\mathbf{9 0 \%}$ Confidence Level

\begin{tabular}{|c|l|c|c|c|}
\hline & & Non-linear Regression & Bootstrap & MLE \\
\hline 1 & Total Points Used & 23 & 23 & 23 \\
\hline 2 & Nominal & $90 \%$ & $90 \%$ & $90 \%$ \\
\hline 3 & Expected Coverage & 21 & 21 & 21 \\
\hline 4 & Covered Points & 21 & 21 & 19 \\
\hline 5 & Average CP (\%) & $92.17 \%$ & $90.14 \%$ & $84.64 \%$ \\
\hline 6 & Average $\mid \mathrm{CP}-90(\%)$ & $2.17 \%$ & $2.35 \%$ & $8.55 \%$ \\
\hline 7 & Range & $91.30-95.65$ & $82.61-95.65$ & $73.91-95.65$ \\
\hline 8 & SD of CPs & 1.80 & 3.06 & 7.86 \\
\hline
\end{tabular}

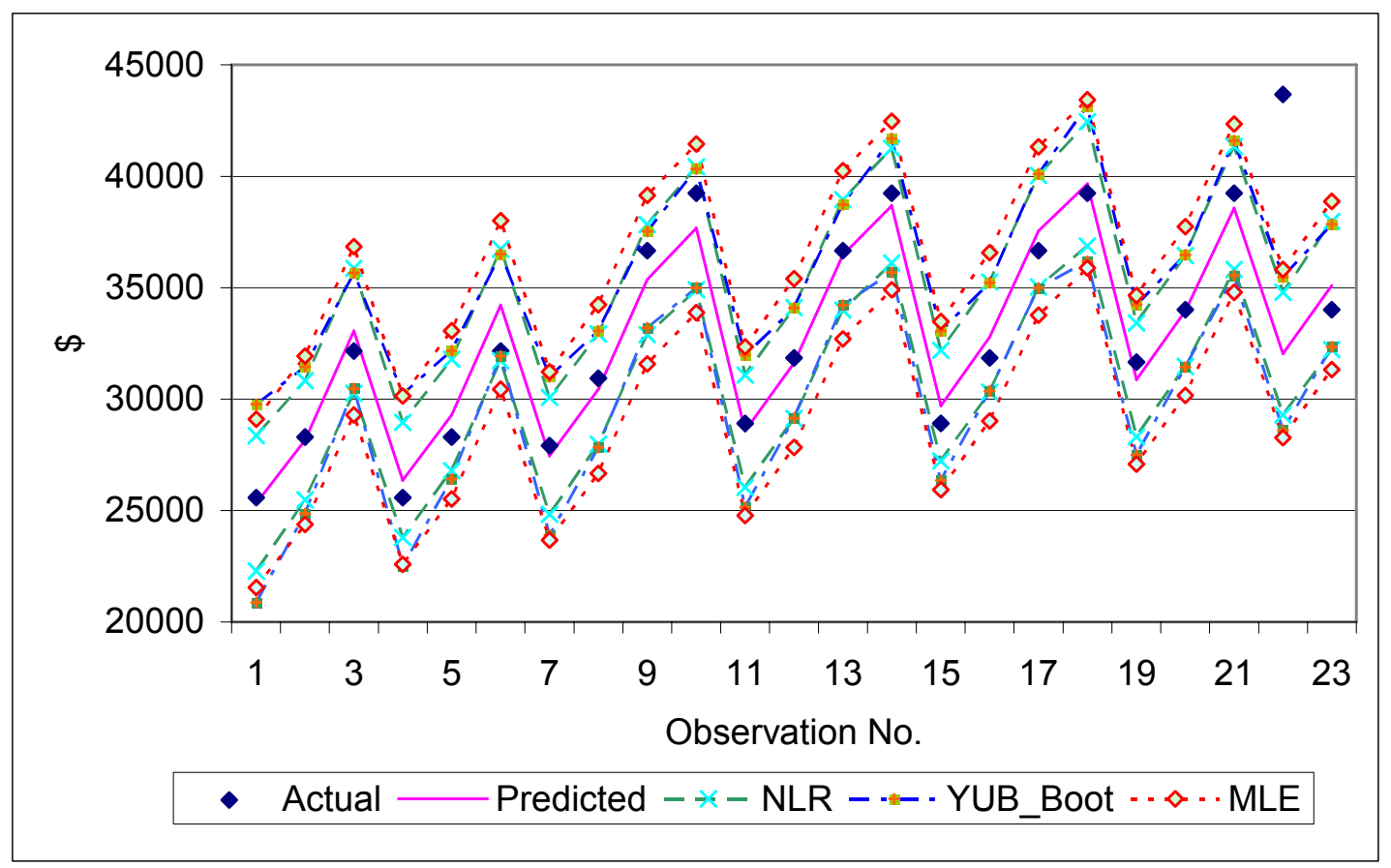

Figure 4.1 Confidence Intervals by all the Techniques for Case B 


\subsubsection{Case C}

The data for Case C were taken from J. G. Carney et.al.[31]. Here, the input vector contains only a single value, and the inputs were uniformly drawn at random from the interval [0, 2]. The target values were generated using (60) and (61). One thousand data were used for training and 1000 were used for testing the models. The net architecture was 1-3-1, which was trained using 200 epochs. This case was complex compared to the other cases; hence an additional hidden neuron was used. Training and confidence interval parameters are given in Table 4.7, and Table 4.8 summarizes the results for the models.

$$
\begin{gathered}
y=4.26\left(\mathrm{e}^{-\mathrm{x}}-4 \mathrm{e}^{-2 \mathrm{x}}+3 \mathrm{e}^{-3 \mathrm{x}}\right)+\varepsilon(\mathrm{x}) \\
\left\langle\varepsilon^{2}(\mathrm{x})\right\rangle=-0.1\left(\mathrm{e}^{-18 \mathrm{x}}-\mathrm{e}^{-4 \mathrm{x}}\right)
\end{gathered}
$$

As it is shown in Table 4.8, bootstrapping calculated better confidence intervals than the NLR and MLE, with average $|\mathrm{CP}-95|$ of $3.80 \%$. The results obtained by the MLE and NLR technique were almost the same with average $|\mathrm{CP}-95|$ of $5.02 \%$ and $4.98 \%$ respectively. Although, bootstrapping provided the best results of the three techniques, it should be noted that bootstrapping requires high computational powers for large data sets, as in this case, which it took approximately 45 minutes to predict confidence intervals for 15 simulations. However, time taken by other techniques is minimal. Results for all the 15 simulations are given in Table C-5 of Appendix C. Results obtained for 90\% nominal probability are given in Table 4.9. When the intervals were calculated using $90 \%$, bootstrap failed to achieve good results with average $\mid$ CP-90| of 7.09\%, and NLR obtained much better average $\mid$ CP-90| of 3.38\%. The results for all simulations are given in Table C- 6 . Confidence curves obtained by all the techniques for the $11^{\text {th }}$ simulation are shown in Figure 4.2.

Table 4.7 Neural Network and Confidence Interval Parameters for Case C

\begin{tabular}{|l|c|}
\hline Number of Observations for Training & 1000 \\
\hline Number of observations for testing & 1000 \\
\hline Number of inputs & 1 \\
\hline Number of Hidden Neurons & 3 \\
\hline Learning Rate & 0.2 \\
\hline Number of iterations & 200 \\
\hline Total Number of parameters & 10 \\
\hline Degrees of Freedom & 990 \\
\hline Activation function between input and hidden & Sigmoid \\
\hline Activation Function Between Hidden And Output & Linear \\
\hline
\end{tabular}


Table 4.8 Results for Case C: Average Taken Over 15 Samples for 95\% Confidence Level

\begin{tabular}{|c|l|c|c|c|}
\hline & & Non-linear Regression & Bootstrap & MLE \\
\hline 1 & Total Points Used & 2000 & 2000 & 2000 \\
\hline 2 & Nominal & $95 \%$ & $95 \%$ & $95 \%$ \\
\hline 3 & Expected Coverage & 1900 & 1900 & 1900 \\
\hline 4 & Covered Points & 1800 & 1973 & 1986 \\
\hline 5 & Average CP $(\%)$ & $90.02 \%$ & $98.64 \%$ & $99.32 \%$ \\
\hline 6 & Average $|\mathrm{CP}-95(\%)|$ & $4.98 \%$ & $3.80 \%$ & $5.02 \%$ \\
\hline 7 & Range & $89.75-90.35$ & $93.85-100.00$ & $89.75-100.00$ \\
\hline 8 & SD of CPs & 0.19 & 1.84 & 2.65 \\
\hline
\end{tabular}

Table 4.9 Results for Case C: Average Taken Over 15 Samples for $90 \%$ Confidence Level

\begin{tabular}{|c|l|c|c|c|}
\hline & & Non-linear Regression & Bootstrap & MLE \\
\hline 1 & Total Points Used & 2000 & 2000 & 2000 \\
\hline 2 & Nominal & $90 \%$ & $90 \%$ & $90 \%$ \\
\hline 3 & Expected Coverage & 1800 & 1800 & 1800 \\
\hline 4 & Covered Points & 1732 & 1942 & 1982 \\
\hline 5 & Average CP $(\%)$ & $86.62 \%$ & $97.09 \%$ & $99.11 \%$ \\
\hline 6 & Average $|\mathrm{CP}-90(\%)|$ & $3.38 \%$ & $7.09 \%$ & $9.55 \%$ \\
\hline 7 & Range & $86.00-87.05$ & $91.05-99.90$ & $86.70-100.00$ \\
\hline 8 & SD of CPs & 0.28 & 2.60 & 3.43 \\
\hline
\end{tabular}




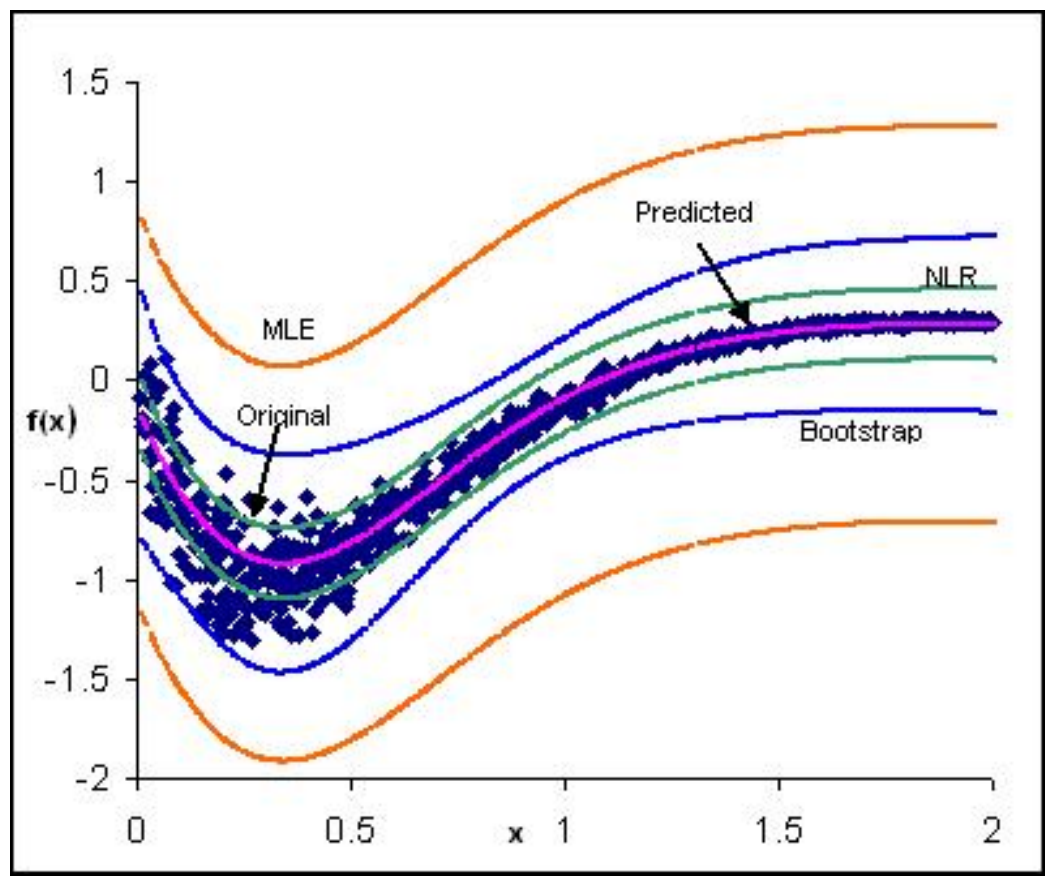

Figure 4.2 Confidence Curves Obtained by all Techniques for Case C at 95\% Confidence Level

It clearly can be seen from Figure 4.2 that MLE greatly overestimated variance and obtained very wide intervals. Bootstrapping also overestimated the confidence intervals, in particular for the values of $\mathrm{x}$ greater than 0.5 , whereas NLR underestimated the confidence level for the values of $\mathrm{x}$ less than 0.8 .

\subsubsection{Case D}

The data for case D was taken from SAS's Website [45] as explained in Section 3.2. There were 104 observations, one output variable, and one input variable in the dataset. The neural network and confidence interval parameters are given in Table 4.10, and the results are summarized in Table 4.11 (95\%) and Table 4.12 (90\%). In this case, bootstrap was better than the NLR and the MLE techniques with $|\mathrm{CP}-95|$ of $4.18 \%$. Results from this case are given in Table C-7 and Table C- 8 of Appendix C. For 90\% nominal probability, again bootstrap was better than NLR and the MLE with |CP-90| of 5.12\%. All of the technique gave relatively poor results for case D compared to the other three cases. 
Table 4.10 Neural Network and Confidence Interval Parameters for Case D

\begin{tabular}{|l|c|}
\hline Number of Observations For Training & 80 \\
\hline Number of Observations For Testing & 24 \\
\hline Number of Inputs & 1 \\
\hline Number of Hidden Neurons & 2 \\
\hline Learning Rate & 0.2 \\
\hline Number of Iterations & 300 \\
\hline Total Number of Parameters & 7 \\
\hline Degrees of Freedom & 73 \\
\hline Activation Function Between Input And Hidden & Sigmoid \\
\hline Activation Function Between Hidden And Output & Linear \\
\hline
\end{tabular}

Table 4.11 Results for Case D: Average Taken Over 15 Samples for 95\% Confidence Level

\begin{tabular}{|c|l|c|c|c|}
\hline & & Non-linear Regression & Bootstrap & MLE \\
\hline 1 & Total Points Used & 104 & 104 & 104 \\
\hline 2 & Nominal & $95 \%$ & $95 \%$ & $95 \%$ \\
\hline 3 & Expected Coverage & 99 & 99 & 99 \\
\hline 4 & Covered Points & 90 & 102 & 97 \\
\hline 5 & Average CP $(\%)$ & $86.73 \%$ & $97.69 \%$ & $93.53 \%$ \\
\hline 6 & Average $\mid \mathrm{CP}-95(\%)$ & $8.27 \%$ & $4.18 \%$ & $6.81 \%$ \\
\hline 7 & Range & $85.58-91.35$ & $88.46-100.00$ & $83.65-100.00$ \\
\hline 8 & SD of CPs & 1.59 & 3.77 & 7.26 \\
\hline
\end{tabular}

Table 4.12 Results for Case D: Average Taken Over 15 Samples for $90 \%$ Confidence Level

\begin{tabular}{|c|l|c|c|c|}
\hline & & Non-linear Regression & Bootstrap & MLE \\
\hline 1 & Total Points Used & 104 & 104 & 104 \\
\hline 2 & Nominal & $90 \%$ & $90 \%$ & $90 \%$ \\
\hline 3 & Expected Coverage & 94 & 94 & 94 \\
\hline 4 & Covered Points & 85 & 97 & 95 \\
\hline 5 & Average CP $(\%)$ & $81.86 \%$ & $93.14 \%$ & $90.96 \%$ \\
\hline 6 & Average $\mid \mathrm{CP}-90(\%)$ & $8.14 \%$ & $5.12 \%$ & $9.71 \%$ \\
\hline 7 & Range & $81.73-83.65$ & $83.65-100.00$ & $77.88-100.00$ \\
\hline 8 & SD of CPs & 0.50 & 4.97 & 10.04 \\
\hline
\end{tabular}




\section{Results and Conclusion}

The non-linear regression, the bootstrapping estimation, and the maximum likelihood estimation were identified as techniques for predicting confidence intervals on the output of the neural networks. These techniques were modeled in Visual Basic to analyze them further by testing on various data sets. All three techniques were tested on artificial and real data sets.

To assess these techniques, the coverage probability was calculated over 15 samples and the average was taken. The average of the absolute values of (CP-95) for all cases are given in Table 5.1 and Figure 5.1. For a nominal probability of $95 \%$, the bootstrapping estimation (3.23\%) was slightly better than the NLR technique (3.67\%), and the NLR was better than the MLE (4.98\%). However the bootstrapping method had significantly higher standard deviations in its estimates than the NLR method. Table 5.2 and Figure 5.2 indicated slightly better results for the bootstrapping method at $|\mathrm{CP}-90 \%|$ when comparing averages but worse results when comparing standard deviations.

On the basis of average coverage probability minus nominal probability, out of the eight cases (95 and 90), NLR and bootstrap were better for four cases. Also, bootstrapping was never the worst of all the techniques in the eight cases. However, when the ranges and standard deviation (SD) of the $\mathrm{CP}$ for all simulations were considered, NLR had the least range SD and was consistent with the coverage probability. Coverage probability ranges for bootstrapping were not consistent, as bootstrapping involves the training of the bootstrap samples, and the coverage probabilities depend upon the training of each sample. The random weight initialization for each training session was always different and caused the variation in the training of each bootstrap sample.

After examining these results, recommending which technique should be used depends on the need of the user. If consistency is the primary criterion, the non-linear regression technique should be used as it had the smallest ranges, and its average $\mathrm{CP}$ values were within one-half percentage point of the bootstrapping method, and this difference was statistically not significant. If coverage probability is the primary criterion, bootstrapping can be used in the instances where the difference between non-linear regression and bootstrap is statistically significant and bootstrap has better $\mathrm{CP}$-nominal probability. T-tests were conducted to test if the average $\mid \mathrm{CP}$ 95| and average |CP-90| obtained by NLR and bootstrap were statistically different, given in Appendix F. For average $|\mathrm{CP}-95|$, for all the cases, the t-statistics were significant at the 0.05 
and 0.1 critical alpha level. Therefore, the null hypothesis for all cases and critical alpha level of 0.05 and 0.1 was rejected, and it was concluded that there was significant difference between the average |CP-95| obtained by NLR and bootstrap, as given in Table F-1. For the average |CP-90|, the null hypothesis was accepted for Case A and Case B and it was rejected for Case $\mathrm{C}$ and Case $\mathrm{D}$, at critical alpha level of 0.05 and 0.1 . Therefore, it was concluded that there was no significant difference in the average |CP-90| obtained by NLR and bootstrap for Case A and Case $\mathrm{B}$, as given Table F-2.

Table 5.1 Values of (CP-95) for Each Case

\begin{tabular}{|c|c|c|c|c|c|c|c|c|}
\hline & \multicolumn{7}{|c|}{ Average |CP-95 (\%)| } \\
\cline { 2 - 7 } & \multicolumn{2}{|c|}{ NLR } & \multicolumn{2}{c|}{ Bootstrap } & \multicolumn{2}{c|}{ MLE } & Lowest & Lowest \\
& Avg & SD & Avg & SD & Avg & SD & CP-95| & SD \\
\hline Case A & $0.76 \%$ & 0.61 & $3.29 \%$ & 3.57 & $3.45 \%$ & 3.14 & NLR & NLR \\
\hline Case B & $0.65 \%$ & 0.00 & $1.67 \%$ & 2.12 & $4.62 \%$ & 3.98 & NLR & NLR \\
\hline Case C & $4.98 \%$ & 0.19 & $3.80 \%$ & 1.84 & $5.02 \%$ & 2.65 & Boot & NLR \\
\hline Case D & $8.27 \%$ & 1.59 & $4.18 \%$ & 3.77 & $6.81 \%$ & 7.26 & Boot & NLR \\
\hline Average & $\mathbf{3 . 6 7 \%}$ & $\mathbf{0 . 6 0}$ & $\mathbf{3 . 2 3} \%$ & $\mathbf{2 . 8 2}$ & $\mathbf{4 . 9 8 \%}$ & $\mathbf{4 . 2 6}$ & Boot & NLR \\
\hline
\end{tabular}

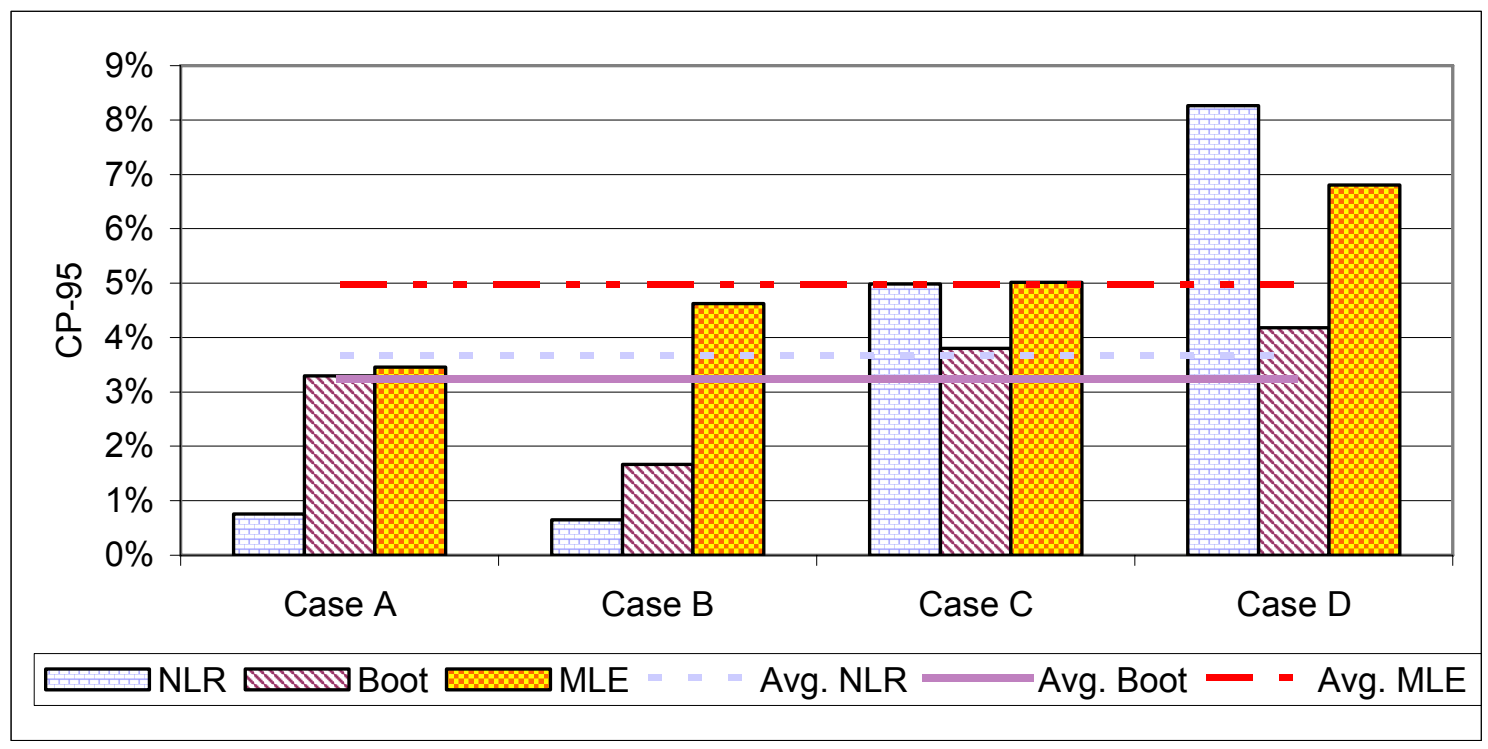

Figure 5.1 CP - 95 Results for Each Technique for Respective Data Sets Shown in this Order: NLR, Bootstrapping, and MLE 
Table 5.2 Values of (CP-90) for Each Case

\begin{tabular}{|c|c|c|c|c|c|c|c|c|}
\hline & \multicolumn{7}{|c|}{ Average |CP-90 (\%)| } \\
\cline { 2 - 8 } & \multicolumn{2}{|c|}{ NLR } & \multicolumn{2}{|c|}{ Bootstrap } & \multicolumn{2}{c|}{ MLE } & $\begin{array}{c}\text { Lowest } \\
\mid \text { CP-90| }\end{array}$ & $\begin{array}{c}\text { Lowest } \\
\text { SD }\end{array}$ \\
\hline Case A & $7.87 \%$ & 1.43 & $6.91 \%$ & 8.48 & $12.51 \%$ & 8.27 & Boot & NLR \\
\hline Case B & $2.17 \%$ & 1.80 & $2.35 \%$ & 3.06 & $8.55 \%$ & 7.86 & NLR & NLR \\
\hline Case C & $3.38 \%$ & 0.28 & $7.09 \%$ & 2.60 & $9.55 \%$ & 3.43 & NLR & NLR \\
\hline Case D & $8.14 \%$ & 0.50 & $5.12 \%$ & 4.97 & $9.71 \%$ & 10.04 & Boot & NLR \\
\hline Average & $\mathbf{5 . 3 9 \%}$ & $\mathbf{1 . 0 0}$ & $\mathbf{5 . 3 7 \%}$ & $\mathbf{4 . 7 8}$ & $\mathbf{1 0 . 0 8} \%$ & 7.40 & Boot & NLR \\
\hline
\end{tabular}

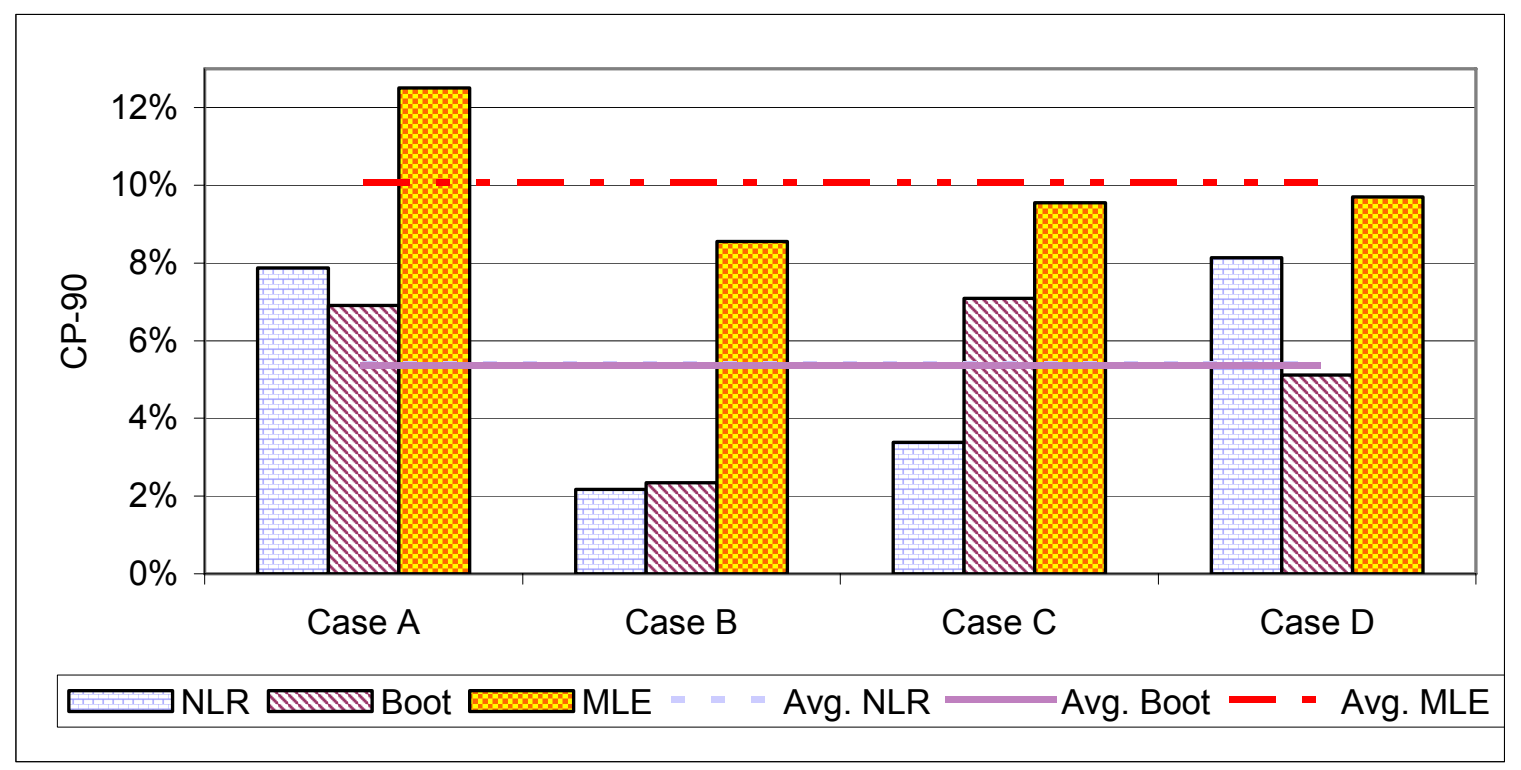

Figure 5.2 CP - 90 Results for Each Technique for Respective Data Sets Shown in this Order: NLR, Bootstrapping and MLE

The time taken for calculation was also a criterion to judge the performance of these models. The situations where the confidence intervals are most important are in mission-critical or safety-critical situations. Therefore, the time needed to calculate the confidence intervals ideally should be small. Bootstrapping estimation took a large amount of time for each run, as it was trained $B$ times, where $B$ is the number of bootstrap samples. The MLE model also took more time than the non-linear regression, as two nets are trained for the calculation of the confidence intervals. The non-linear regression was the fastest, as it is trained only once, and it 
obtained more consistent results in all cases and was the best method in one-half of the cases studied.

\subsection{Future Study}

The focus of this research was to model, analyze, and compare three popular confidence estimation techniques on the output of neural networks. This research was limited to four cases studied for each technique. The following future studies are suggested for this research:

1. If possible, refine the non-linear regression technique so that the problem of singular matrix or a large matrix does not occur.

2. Develop a better methodology than the coverage probability to compare the results calculated by the confidence estimation techniques, as CP involves using different nominal probabilities to compare the techniques.

3. Develop a rationale for choosing the training parameters for the $\mathrm{NN}$ with residuals as target values for the maximum likelihood estimation as,

4. Determine the effect of more hidden neurons in a layer and more layers upon the output of the network.

The most critical problem faced in this research was of the computation of the inverse of the Jacobian matrix; hence, if possible, refinement of the existing non-linear regression technique should be done. Different nominal probabilities (95\% and 90\%) were used in this research to compare the techniques using the coverage probability technique, which demands extra time and efforts. Therefore, a better method to compare the techniques in a single run would save time and effort. 


\section{References}

1. X. Yao and Y. Liu, "Neural networks for breast cancer diagnosis," Proceedings of the 1999 Congress on Evolutionary Computation, pp. 1760-1767, IEEE Press, New York, NY, USA, July 1999.

2. E. Smith and A. K. Mason, "Cost estimation predictive modeling: Regression versus neural network,", The Engineering Economist, vol. 42, no. 2, pp. 137-161, Winter 1997.

3. M. Scardi, "Empirical models in ecology: some applications of artificial neural networks", VII International Congress of Ecology, July 1998.

4. Encyclopædia $\odot$ Britannica, Inc., http://www.britannica.com/, 2004.

5. Laurene Fausett, Fundamentals of Neural Networks: Architectures, Algorithms, and Applications, New Jersey: Prentice-Hall, pp 5-7, 1994.

6. Data \& Analysis Center for Software's (DACS) Artificial Neural Networks Technology Web page, http://www.dacs.dtic.mil/techs/neural/neural4.html, 1992.

7. Laurene Fausett, Fundamentals of Neural Networks: Architectures, Algorithms, and Applications, New Jersey: Prentice-Hall, pp 22-24, 1994.

8. Saint Louis University's Web page on Neural Networks, http://hem.hj.se/ de96klda/NeuralNetworks.htm, November 1998.

9. Mathworks' Neural Network Toolbox Documentation, http://www.mathworks.com/access/helpdesk/help/toolbox/nnet/percept8.shtml\#14820, 2004.

10. West Virginia University's Course Web page on Neural Networks, http://www.csee.wvu.edu/classes/cpe320/presentations/Intro-ArtificialNeural.pdf, pp 27, 2000.

11. Mathworks' Neural Network Toolbox Documentation, http://www.mathworks.com/access/helpdesk/help/toolbox/nnet/backpr5.shtml, 2004.

12. S. M. Weiss, C.A. Kulikowski, Computer Systems That Learn, Morgan Kaufmann, San Mateo, 1991.

13. R.D. De Veaux, J.Schumi, J. Schweinsberg, and L.H. Ungar, Prediction intervals for neural networks via nonlinear regression, Technometrics, 1998, 40, 273-282. 
14. R. Shao, E. B. Martin, J. Zhang, and A. J. Morris, Confidence bounds for neural network representations, Computers \& Chemical Engineering, 1997, Vol. 21(SS), S1173-S1178.

15. L.Yang, T. Kavli, M. Carlin, S. Clausen and P. F. M. de Groot, An evaluation of confidence bound estimation methods for neural networks, Proceedings of European Symposium Intelligent Techniques, 2000, pp 322-329.

16. G. Chryssolouris, M. Lee, and A. Ramsey, IEEE Transactions on Neural Networks, v 7, n 1, Jan, 1996, pp 229-232.

17. L. H. Ungar, R. D. D. Veaux, and E. Rosengarten. Estimating prediction intervals for artificial neural networks, Proceedings of the 9th Yale Workshop on Adaptive and Learning Systems, 1996.

18. I. Rivals and L. Personnaz, Construction of confidence intervals for neural networks based on least squares estimation, Neural Networks, v 13, n 4, May, 2000, pp 463-484

19. J. E. Freud and R. E. Walpole, Mathematical Statistics, Englewood Cliffs, New Jersey: Prentice-Hall, 1987.

20. B. Efron and R. J. Tibshirani, An introduction to the bootstrap, New York: Chapman and Hall, 1993.

21. K. N. Lankalpalli and R. C. Creese, Cost estimating using neural networks, Transactions of 13th International Cost Engineering Congress, Association of Cost Engineers, 1994, pp CE17.1-CE17.15.

22. W. G. Baxt and H. White, Bootstrapping confidence intervals for clinical input variable effects in network trained to identify the presence of acute myocardial infarction, Neural Computation, 7, pp 624-638.

23. G. Papadopoulos, P. J. Edwards, and A. F. Murray, Confidence estimation methods for neural networks: A practical comparison, IEEE Transactions on Neural Networks, v 12, n 6, November, 2001, pp 1278-1287.

24. R. Tibshirani, A comparison of some error estimates for neural network models, Neural Computation, v 8, n 1, Jan, 1996, pp 152-163.

25. R. Dybowski and S. Roberts, Confidence intervals and prediction intervals for feedforward neural networks, Clinical Applications of Artificial Neural Networks, Cambridge: Cambridge University Press, 2001, pp 298-326. 
26. R.E. Walpole, R.H. Myers, and S.L. Myers, Probability and statistics for engineers and scientists, New Jersey: Prentice-Hall, 1998.

27. J. Schumann, P. Gupta and S. Nelson, On verification \& validation of neural network based controllers, Proceedings of Engineering Applications of Neural Networks (EANN '03), September, 2003.

28. V. J. Easton and J. H. McColl, http://www.cas.lancs.ac.uk/glossary_v1.1/confint.html, Online Statistics Glossary v1.1, 2004.

29. D. Lowe and K. Zapart, Point-wise confidence interval estimation by neural networks: A comparative study based on automotive engine calibration, Neural Computation Application, v 8, no. 1, pp. 77-85, 1999.

30. T. Heskes, Practical confidence and prediction intervals, Advances in Neural Information Processing Systems, 9, 176-182, 1997.

31. J. G. Carney, P. Cunningham and U. Bhagwan, Confidence and prediction intervals for neural network ensembles, In Proceedings of the International Joint Conference on Neural Networks 1999, paper 2090, 1999.

32. R. Dybowski, Assigning Confidence intervals to neural networks Predictions, Technical Report, Division of Infection (St Thomas' Hospital), King's College, London, March, 1997.

33. J. R. Donaldson and R. B. Scnabel, Computational experience with confidence regions and confidence intervals for nonlinear least squares, Technometrics, vol. 29, pp. 67-82, 1987.

34. J. T. G. Hwang and A. A. Ding, Prediction intervals for artificial neural networks, Journal of American Statistical Association, vol. 92, no 438, pp. 748-757, June, 1997.

35. H. Haraldsson, L. Edenbrandt, and M. Ohlsson, Detecting acute myocardial infarction in the 12-lead ECG using Hermite expansions and neural networks, Artificial Intelligence in Medicine, vol. 32, pp. 127-136, 2004.

36. S. N. Londhe and M. C. Deo, Artificial neural networks for wave propagation, Journal of Coastal Research, vol. 20, no 4, pp. 1061-1069, 2004.

37. A. Silva and L. Moulin, Confidence intervals for neural network based short-term load forecasting, IEEE Transactions On Power Systems, vol. 15, no. 4, pp 1191 - 1196, 2000. 
38. C. Hwang, K. Seok, and D. Cho, A prediction interval estimation method for KMSE, ICNC, LNCS 3610, pp. 536-545, 2005.

39. I. Rivals and L. Personnaz, Jacobian conditioning analysis for model validation, Neural Computation, vol. 16, pp 401-418, 2004.

40. I. Rivals and L. Personnaz ,Construction of confidence intervals in Neural Modeling using a linear Taylor Expansion, Proceedings of the International workshop on Advanced Black Box Techniques for Nonlinear Modeling, July, p 463-84, 1998.

41. S. L. Ho, M. Xie, L. C. Tang, K. Xu, and T. N. Goh, Neural Network Modeling With Confidence Bounds: A Case Study on the Solder Paste Deposition Process, IEEE Transactions On Electronics Packaging Manufacturing, Vol. 24, No. 4, pp 323 - 332, October, 2001.

42. V. M. Bhide and M. J. Piovoso, Statistics on reliability of neural network estimates, Proceedings of the American Control Conference, pp. 1877-1881, June, 1995.

43. W. Y. Goh, C. P. Lim, K. K. Peh and K. Subari, Application of a recurrent neural network to prediction of drug dissolution profiles, Neural Computing and Applications, vol. 10, pp. 311-317, 2002.

44. D. Nguyen and B. Widrow, Improving the learning speed of 2-layer neural networks by choosing initial values of the adaptive weights, Proceedings of the International Joint Conference on Neural Networks, vol. 3, pp. 21-26, 1990.

45. J. Neter, W. Wasserman, and M. Kutner, Applied Linear Regression Models, Irwin Publications, $2^{\text {nd }}$ edition, pp 82-83, 1989.

46. R. A. McKim, Neural Network Applications to Cost Engineering, Cost Engineering, pp 31-35, July 1993.

47. SAS's Sample Data Sets-Tree Data, http://support.sas.com/training/forms/hecftp1.html 48. National Institute of Standards and Technology (NIST), Online Engineering Statistics Handbook, Two-Sample t-Test for Equal Means, http://www.itl.nist.gov/div898/handbook/eda/section3/eda353.htm 


\section{Appendix A Data Tables}

Table A-1 The Data Used for Developing Neural Network in Section 3.2.1-

\begin{tabular}{|c|c|c|c|c|c|c|}
\hline S.N. & $\mathbf{x}_{1}$ & $\mathbf{x}_{2}$ & $\mathbf{x}_{3}$ & $\mathbf{x}_{4}$ & $x_{5}$ & Actual y \\
\hline 1 & 0.0239 & 0.2281 & 0.2837 & 0.9457 & 0.6764 & 4.8494 \\
\hline 2 & 0.1571 & 0.5192 & 0.5285 & 0.7370 & 0.6364 & 5.9876 \\
\hline 3 & 0.6795 & 0.6443 & 0.8955 & 0.3714 & 0.9198 & 9.5968 \\
\hline 4 & 0.9631 & 0.2735 & 0.3764 & 0.1937 & 0.7892 & 7.0193 \\
\hline 5 & 0.8691 & 0.4995 & 0.4351 & 0.0631 & 0.7287 & 6.7905 \\
\hline 6 & 0.4740 & 0.4804 & 0.8204 & 0.6564 & 0.9313 & 8.5989 \\
\hline 7 & 0.1256 & 0.6800 & 0.1080 & 0.0453 & 0.2924 & 2.7894 \\
\hline 8 & 0.3469 & 0.6336 & 0.5620 & 0.1417 & 0.6948 & 6.4071 \\
\hline 9 & 0.1467 & 0.5378 & 0.8104 & 0.5658 & 0.0556 & 4.7262 \\
\hline 10 & 0.7358 & 0.8559 & 0.8393 & 0.5282 & 0.7486 & 10.0420 \\
\hline 11 & 0.2762 & 0.1367 & 0.7559 & 0.4707 & 0.8531 & 7.2822 \\
\hline 12 & 0.6781 & 0.7725 & 0.7578 & 0.2065 & 0.5496 & 7.9863 \\
\hline 13 & 0.6267 & 0.0449 & 0.0119 & 0.0594 & 0.7131 & 3.9293 \\
\hline 14 & 0.1339 & 0.3106 & 0.5082 & 0.2049 & 0.7556 & 5.6334 \\
\hline 15 & 0.5132 & 0.1146 & 0.3071 & 0.9322 & 0.2545 & 3.7428 \\
\hline 16 & 0.2137 & 0.3644 & 0.9563 & 0.6002 & 0.1824 & 5.5661 \\
\hline 17 & 0.0397 & 0.3595 & 0.8112 & 0.2297 & 0.4008 & 5.4701 \\
\hline 18 & 0.8914 & 0.8178 & 0.4779 & 0.0551 & 0.2130 & 6.2285 \\
\hline 19 & 0.5203 & 0.2147 & 0.8441 & 0.1063 & 0.3700 & 5.7536 \\
\hline 20 & 0.9312 & 0.3654 & 0.3229 & 0.6691 & 0.8939 & 7.5658 \\
\hline 21 & 0.1268 & 0.6412 & 0.7021 & 0.3286 & 0.2128 & 5.0560 \\
\hline 22 & 0.4048 & 0.7625 & 0.6609 & 0.7150 & 0.8542 & 8.5634 \\
\hline 23 & 0.8937 & 0.7554 & 0.8679 & 0.8709 & 0.0785 & 7.6090 \\
\hline 24 & 0.8071 & 0.4231 & 0.3670 & 0.3018 & 0.0260 & 3.6514 \\
\hline 25 & 0.0426 & 0.7968 & 0.3431 & 0.7370 & 0.6933 & 6.5253 \\
\hline 26 & 0.2718 & 0.4407 & 0.4416 & 0.7126 & 0.2619 & 4.0625 \\
\hline 27 & 0.2627 & 0.9230 & 0.3075 & 0.0553 & 0.0480 & 4.1544 \\
\hline 28 & 0.7823 & 0.5018 & 0.7328 & 0.8124 & 0.1230 & 5.9276 \\
\hline 29 & 0.0192 & 0.7359 & 0.1902 & 0.0783 & 0.1991 & 3.0333 \\
\hline 30 & 0.8913 & 0.8169 & 0.8083 & 0.0748 & 0.3980 & 8.3226 \\
\hline 31 & 0.0134 & 0.6790 & 0.7415 & 0.8339 & 0.3749 & 6.3185 \\
\hline
\end{tabular}


Table A-1 The Data Used for Developing Neural Network in Section 3.2.1-Cont'd

\begin{tabular}{|c|c|c|c|c|c|c|}
\hline S.N. & $\mathbf{x}_{1}$ & $\mathbf{x}_{2}$ & $\mathbf{x}_{3}$ & $\mathbf{x}_{4}$ & $\mathbf{x}_{5}$ & Actual y \\
\hline 32 & 0.0734 & 0.4884 & 0.9802 & 0.7821 & 0.7508 & 8.1685 \\
\hline 33 & 0.3949 & 0.8804 & 0.2933 & 0.1133 & 0.6062 & 6.2937 \\
\hline 34 & 0.7339 & 0.4585 & 0.7351 & 0.4932 & 0.7389 & 7.9643 \\
\hline 35 & 0.7656 & 0.0137 & 0.6070 & 0.7290 & 0.0064 & 4.4799 \\
\hline 36 & 0.3444 & 0.7594 & 0.2260 & 0.4149 & 0.7128 & 5.9503 \\
\hline 37 & 0.8015 & 0.3532 & 0.9486 & 0.2659 & 0.2815 & 6.8527 \\
\hline 38 & 0.6184 & 0.8659 & 0.5598 & 0.7140 & 0.0068 & 5.8240 \\
\hline 39 & 0.6136 & 0.6239 & 0.7199 & 0.4452 & 0.5346 & 7.1668 \\
\hline 40 & 0.0948 & 0.3264 & 0.4926 & 0.1900 & 0.0998 & 2.9277 \\
\hline 41 & 0.1180 & 0.9481 & 0.2577 & 0.8953 & 0.1227 & 5.0521 \\
\hline 42 & 0.3469 & 0.4105 & 0.0513 & 0.0285 & 0.5911 & 3.1869 \\
\hline 43 & 0.5559 & 0.9643 & 0.4943 & 0.6764 & 0.5592 & 8.3444 \\
\hline 44 & 0.9051 & 0.6873 & 0.2058 & 0.6894 & 0.1003 & 4.6675 \\
\hline 45 & 0.3950 & 0.5173 & 0.3644 & 0.2676 & 0.2310 & 3.6262 \\
\hline 46 & 0.6230 & 0.2508 & 0.2118 & 0.9758 & 0.1386 & 3.2128 \\
\hline 47 & 0.4411 & 0.8772 & 0.3807 & 0.6505 & 0.4002 & 6.3442 \\
\hline 48 & 0.7975 & 0.4668 & 0.0856 & 0.5023 & 0.6999 & 5.4278 \\
\hline 49 & 0.8092 & 0.3320 & 0.9264 & 0.3814 & 0.4807 & 7.6654 \\
\hline 50 & 0.2221 & 0.1583 & 0.6054 & 0.1641 & 0.2355 & 3.8794 \\
\hline 51 & 0.9471 & 0.4417 & 0.4604 & 0.8783 & 0.1391 & 5.3875 \\
\hline 52 & 0.9126 & 0.8013 & 0.9857 & 0.7792 & 0.0232 & 8.1276 \\
\hline 53 & 0.9093 & 0.9862 & 0.7828 & 0.5770 & 0.9999 & 12.4218 \\
\hline 54 & 0.5680 & 0.7294 & 0.1067 & 0.3431 & 0.6226 & 5.3126 \\
\hline 55 & 0.5209 & 0.9931 & 0.0804 & 0.4290 & 0.4198 & 6.1361 \\
\hline 56 & 0.6801 & 0.8100 & 0.2017 & 0.9051 & 0.4014 & 5.8832 \\
\hline 57 & 0.3100 & 0.9004 & 0.8872 & 0.8204 & 0.1096 & 7.2752 \\
\hline 58 & 0.6777 & 0.1640 & 0.1855 & 0.3466 & 0.4621 & 4.1113 \\
\hline 59 & 0.9112 & 0.1917 & 0.6855 & 0.0547 & 0.4423 & 6.4267 \\
\hline 60 & 0.3117 & 0.9877 & 0.4843 & 0.3650 & 0.3965 & 7.2122 \\
\hline 61 & 0.2280 & 0.0467 & 0.1026 & 0.9404 & 0.2700 & 2.5646 \\
\hline 62 & 0.2951 & 0.2270 & 0.1909 & 0.5251 & 0.3857 & 3.2405 \\
\hline 63 & 0.2512 & 0.6615 & 0.3822 & 0.5652 & 0.3585 & 4.7090 \\
\hline 64 & 0.1628 & 0.0218 & 0.7591 & 0.9421 & 0.4239 & 5.7553 \\
\hline 65 & 0.8647 & 0.4620 & 0.3933 & 0.3864 & 0.4532 & 5.7985 \\
\hline
\end{tabular}


Table A-1 The Data Used for Developing Neural Network in Section 3.2.1-Cont'd

\begin{tabular}{|c|c|c|c|c|c|c|}
\hline S.N. & $\mathbf{x}_{1}$ & $\mathbf{x}_{2}$ & $\mathbf{x}_{3}$ & $\mathbf{x}_{4}$ & $\mathbf{x}_{5}$ & Actual y \\
\hline 66 & 0.6335 & 0.5478 & 0.6324 & 0.0639 & 0.1765 & 4.7845 \\
\hline 67 & 0.9811 & 0.5597 & 0.4834 & 0.0639 & 0.8187 & 7.9126 \\
\hline 68 & 0.7080 & 0.1566 & 0.9612 & 0.2189 & 0.7393 & 8.2838 \\
\hline 69 & 0.7386 & 0.4789 & 0.0463 & 0.9596 & 0.2925 & 3.7556 \\
\hline 70 & 0.3404 & 0.3797 & 0.9975 & 0.2679 & 0.9131 & 8.5558 \\
\hline 71 & 0.8566 & 0.7577 & 0.0272 & 0.2721 & 0.2793 & 4.5204 \\
\hline 72 & 0.3696 & 0.9260 & 0.0232 & 0.5718 & 0.0189 & 3.5792 \\
\hline 73 & 0.5100 & 0.5620 & 0.6927 & 0.3615 & 0.0258 & 4.5278 \\
\hline 74 & 0.2969 & 0.0253 & 0.6400 & 0.3067 & 0.7892 & 6.4468 \\
\hline 75 & 0.8339 & 0.9618 & 0.6412 & 0.3240 & 0.0971 & 7.5821 \\
\hline 76 & 0.0684 & 0.6194 & 0.6557 & 0.8244 & 0.5986 & 6.6475 \\
\hline 77 & 0.9727 & 0.5761 & 0.1997 & 0.7980 & 0.3587 & 5.5930 \\
\hline 78 & 0.6869 & 0.8771 & 0.3365 & 0.1055 & 0.4638 & 6.4941 \\
\hline 79 & 0.3473 & 0.9077 & 0.0337 & 0.2185 & 0.3502 & 4.4881 \\
\hline 80 & 0.3200 & 0.3063 & 0.7559 & 0.1178 & 0.4499 & 5.4575 \\
\hline 81 & 0.3940 & 0.7924 & 0.5717 & 0.1294 & 0.6936 & 7.2240 \\
\hline 82 & 0.6238 & 0.6501 & 0.5776 & 0.3082 & 0.2359 & 5.4118 \\
\hline 83 & 0.8650 & 0.0365 & 0.6956 & 0.2370 & 0.9106 & 8.4079 \\
\hline 84 & 0.2149 & 0.4066 & 0.5051 & 0.2656 & 0.8267 & 6.1368 \\
\hline 85 & 0.1775 & 0.6390 & 0.3491 & 0.9226 & 0.2894 & 4.3601 \\
\hline 86 & 0.2020 & 0.0233 & 0.9229 & 0.7632 & 0.2136 & 5.5011 \\
\hline 87 & 0.9238 & 0.3293 & 0.1232 & 0.6243 & 0.3323 & 4.4262 \\
\hline 88 & 0.2562 & 0.3846 & 0.6441 & 0.5276 & 0.2604 & 4.6464 \\
\hline 89 & 0.9465 & 0.6304 & 0.5181 & 0.9919 & 0.1338 & 6.1468 \\
\hline 90 & 0.5620 & 0.3794 & 0.9842 & 0.6549 & 0.3658 & 7.0046 \\
\hline 91 & 0.8507 & 0.8384 & 0.8734 & 0.1001 & 0.5907 & 9.3880 \\
\hline 92 & 0.1984 & 0.5782 & 0.4140 & 0.5404 & 0.8026 & 6.2603 \\
\hline 93 & 0.6868 & 0.8578 & 0.3759 & 0.7524 & 0.7593 & 8.2448 \\
\hline 94 & 0.2944 & 0.9174 & 0.6105 & 0.1310 & 0.6146 & 7.7518 \\
\hline 95 & 0.1202 & 0.3986 & 0.6082 & 0.2452 & 0.3633 & 4.6000 \\
\hline 96 & 0.3092 & 0.1226 & 0.3854 & 0.3724 & 0.5265 & 4.4548 \\
\hline 97 & 0.1859 & 0.4205 & 0.6849 & 0.9640 & 0.4316 & 5.7401 \\
\hline 98 & 0.9842 & 0.2275 & 0.4219 & 0.2407 & 0.9888 & 8.1059 \\
\hline 99 & 0.2862 & 0.3026 & 0.6762 & 0.1097 & 0.5313 & 5.4082 \\
\hline
\end{tabular}


Table A-1 The Data Used for Developing Neural Network in Section 3.2.1-Cont'd

\begin{tabular}{|c|c|c|c|c|c|c|}
\hline S.N. & $\mathbf{x}_{1}$ & $\mathbf{x}_{2}$ & $\mathbf{x}_{3}$ & $\mathbf{x}_{4}$ & $\mathbf{x}_{5}$ & Actual y \\
\hline 100 & 0.3964 & 0.1493 & 0.4643 & 0.7676 & 0.9578 & 6.8887 \\
\hline 101 & 0.0071 & 0.8208 & 0.8392 & 0.5430 & 0.0840 & 6.0881 \\
\hline 102 & 0.8158 & 0.3934 & 0.9404 & 0.4607 & 0.2343 & 6.8912 \\
\hline 103 & 0.2749 & 0.0008 & 0.5066 & 0.9608 & 0.4835 & 5.0919 \\
\hline 104 & 0.4890 & 0.1120 & 0.6724 & 0.8636 & 0.2614 & 5.1469 \\
\hline 105 & 0.1810 & 0.9914 & 0.3332 & 0.9942 & 0.6214 & 7.8042 \\
\hline 106 & 0.1079 & 0.8806 & 0.4427 & 0.5065 & 0.3362 & 5.8988 \\
\hline 107 & 0.8181 & 0.6815 & 0.8889 & 0.2395 & 0.3648 & 7.7920 \\
\hline 108 & 0.5097 & 0.3612 & 0.8775 & 0.0385 & 0.4585 & 6.2014 \\
\hline 109 & 0.4348 & 0.2717 & 0.5575 & 0.5584 & 0.8244 & 6.7130 \\
\hline 110 & 0.3425 & 0.9309 & 0.8669 & 0.8539 & 0.3047 & 8.2653 \\
\hline 111 & 0.7452 & 0.0928 & 0.5085 & 0.5488 & 0.3653 & 5.3491 \\
\hline 112 & 0.3319 & 0.0906 & 0.4034 & 0.2624 & 0.8145 & 5.6063 \\
\hline 113 & 0.0407 & 0.5712 & 0.6190 & 0.0699 & 0.4435 & 5.0764 \\
\hline 114 & 0.6785 & 0.6325 & 0.0723 & 0.3515 & 0.0855 & 2.9040 \\
\hline 115 & 0.1224 & 0.7119 & 0.2399 & 0.6785 & 0.5409 & 5.0595 \\
\hline 116 & 0.0773 & 0.5078 & 0.5643 & 0.6446 & 0.7030 & 6.2772 \\
\hline 117 & 0.6357 & 0.0396 & 0.6937 & 0.1212 & 0.2191 & 4.8075 \\
\hline 118 & 0.7104 & 0.2313 & 0.2838 & 0.6047 & 0.7401 & 5.9198 \\
\hline 119 & 0.7296 & 0.0281 & 0.1633 & 0.5545 & 0.5701 & 4.7427 \\
\hline 120 & 0.7405 & 0.3688 & 0.1491 & 0.8700 & 0.8939 & 6.3522 \\
\hline 121 & 0.6587 & 0.4608 & 0.6283 & 0.4940 & 0.7414 & 7.3429 \\
\hline 122 & 0.6300 & 0.8905 & 0.1533 & 0.7308 & 0.2405 & 5.3428 \\
\hline 123 & 0.4181 & 0.1814 & 0.8571 & 0.9921 & 0.3026 & 6.0025 \\
\hline 124 & 0.4075 & 0.0294 & 0.4287 & 0.5155 & 0.5689 & 5.0405 \\
\hline 125 & 0.3004 & 0.7691 & 0.7138 & 0.2311 & 0.1105 & 5.3233 \\
\hline 126 & 0.4735 & 0.2910 & 0.8175 & 0.2037 & 0.1535 & 4.8579 \\
\hline 127 & 0.9722 & 0.9219 & 0.8917 & 0.6238 & 0.4208 & 10.2802 \\
\hline 128 & 0.4668 & 0.7926 & 0.5638 & 0.5911 & 0.6917 & 7.7209 \\
\hline 129 & 0.8029 & 0.9061 & 0.1782 & 0.7836 & 0.4943 & 7.0969 \\
\hline 130 & 0.1191 & 0.7769 & 0.7423 & 0.6156 & 0.7220 & 8.0770 \\
\hline 131 & 0.4114 & 0.2153 & 0.7511 & 0.6300 & 0.5954 & 6.5481 \\
\hline 132 & 0.7276 & 0.7320 & 0.7102 & 0.6282 & 0.4134 & 7.5220 \\
\hline 133 & 0.2569 & 0.3297 & 0.6233 & 0.9762 & 0.2045 & 4.5389 \\
\hline
\end{tabular}


Table A-1 The Data Used for Developing Neural Network in Section 3.2.1-Cont'd

\begin{tabular}{|c|c|c|c|c|c|c|}
\hline S.N. & $\mathbf{x}_{1}$ & $\mathbf{x}_{2}$ & $\mathbf{x}_{3}$ & $\mathbf{x}_{4}$ & $\mathbf{x}_{5}$ & Actual y \\
\hline 134 & 0.5201 & 0.7981 & 0.5460 & 0.4931 & 0.7296 & 7.8709 \\
\hline 135 & 0.0844 & 0.9684 & 0.5018 & 0.4287 & 0.0943 & 5.7775 \\
\hline 136 & 0.1304 & 0.2845 & 0.4544 & 0.2136 & 0.9012 & 5.9878 \\
\hline 137 & 0.7163 & 0.7403 & 0.5449 & 0.2579 & 0.0409 & 5.0939 \\
\hline 138 & 0.6686 & 0.5257 & 0.3850 & 0.0464 & 0.3138 & 4.3407 \\
\hline 139 & 0.2552 & 0.6929 & 0.9831 & 0.6398 & 0.7074 & 8.6901 \\
\hline 140 & 0.2494 & 0.6695 & 0.7755 & 0.9577 & 0.7273 & 8.0145 \\
\hline 141 & 0.0931 & 0.6636 & 0.1045 & 0.3358 & 0.1434 & 2.4649 \\
\hline 142 & 0.5527 & 0.4078 & 0.4254 & 0.7132 & 0.7351 & 6.3009 \\
\hline 143 & 0.8479 & 0.1602 & 0.6971 & 0.0533 & 0.1590 & 5.1055 \\
\hline 144 & 0.1886 & 0.3155 & 0.2074 & 0.5268 & 0.1944 & 2.4988 \\
\hline 145 & 0.8582 & 0.0152 & 0.3994 & 0.2757 & 0.4730 & 5.4876 \\
\hline 146 & 0.5354 & 0.5035 & 0.1505 & 0.1208 & 0.4132 & 3.5587 \\
\hline 147 & 0.5329 & 0.6784 & 0.6925 & 0.5082 & 0.2088 & 5.8226 \\
\hline 148 & 0.5740 & 0.5394 & 0.2210 & 0.5600 & 0.9368 & 6.5090 \\
\hline 149 & 0.2175 & 0.2864 & 0.3200 & 0.2001 & 0.9305 & 5.6145 \\
\hline 150 & 0.5359 & 0.1086 & 0.3636 & 0.1453 & 0.2174 & 3.2833 \\
\hline 151 & 0.0119 & 0.4085 & 0.4363 & 0.2094 & 0.7796 & 5.5261 \\
\hline 152 & 0.7644 & 0.1965 & 0.1484 & 0.7671 & 0.8295 & 5.9789 \\
\hline 153 & 0.0674 & 0.2795 & 0.3926 & 0.0248 & 0.1376 & 2.3531 \\
\hline 154 & 0.6001 & 0.7814 & 0.7365 & 0.2785 & 0.6445 & 8.2034 \\
\hline 155 & 0.5402 & 0.8785 & 0.0665 & 0.5590 & 0.7503 & 6.6326 \\
\hline 156 & 0.7044 & 0.9223 & 0.4359 & 0.4430 & 0.6627 & 8.4063 \\
\hline 157 & 0.6319 & 0.2204 & 0.9289 & 0.5939 & 0.5202 & 7.3974 \\
\hline 158 & 0.6073 & 0.4399 & 0.0490 & 0.4452 & 0.9869 & 5.8036 \\
\hline 159 & 0.1536 & 0.9651 & 0.4113 & 0.2516 & 0.0902 & 5.2514 \\
\hline 160 & 0.1905 & 0.7917 & 0.5573 & 0.5785 & 0.0322 & 4.6798 \\
\hline 161 & 0.0420 & 0.8044 & 0.0564 & 0.9220 & 0.9976 & 6.7411 \\
\hline 162 & 0.7442 & 0.6157 & 0.9454 & 0.5453 & 0.5918 & 8.6949 \\
\hline 163 & 0.4474 & 0.9662 & 0.9673 & 0.0853 & 0.7087 & 10.1020 \\
\hline 164 & 0.1288 & 0.5301 & 0.2301 & 0.5638 & 0.5983 & 4.5445 \\
\hline 165 & 0.4196 & 0.8213 & 0.2681 & 0.8713 & 0.3679 & 5.4915 \\
\hline 166 & 0.6343 & 0.2961 & 0.9048 & 0.0549 & 0.1813 & 5.4612 \\
\hline 167 & 0.1907 & 0.4504 & 0.2713 & 0.5807 & 0.6317 & 4.7210 \\
\hline
\end{tabular}


Table A-1 The Data Used for Developing Neural Network in Section 3.2.1-Cont'd

\begin{tabular}{|c|c|c|c|c|c|c|}
\hline S.N. & $x_{1}$ & $\mathbf{x}_{2}$ & $\mathbf{x}_{3}$ & $\mathbf{x}_{4}$ & $x_{5}$ & Actual y \\
\hline 168 & 0.5135 & 0.1835 & 0.5221 & 0.2426 & 0.2151 & 3.9876 \\
\hline 169 & 0.9579 & 0.9167 & 0.3560 & 0.3826 & 0.9830 & 10.1209 \\
\hline 170 & 0.2054 & 0.9116 & 0.2765 & 0.5485 & 0.6124 & 6.6535 \\
\hline 171 & 0.8182 & 0.3308 & 0.4105 & 0.8696 & 0.3685 & 5.4959 \\
\hline 172 & 0.9750 & 0.8725 & 0.6025 & 0.1045 & 0.8472 & 10.0155 \\
\hline 173 & 0.3113 & 0.2304 & 0.3960 & 0.7194 & 0.1274 & 3.1724 \\
\hline 174 & 0.4886 & 0.0289 & 0.0229 & 0.3573 & 0.7044 & 3.9847 \\
\hline 175 & 0.2581 & 0.1801 & 0.6694 & 0.6382 & 0.6542 & 6.2441 \\
\hline 176 & 0.1128 & 0.6585 & 0.0555 & 0.4213 & 0.5148 & 3.8123 \\
\hline 177 & 0.1402 & 0.2739 & 0.8277 & 0.3332 & 0.9574 & 7.8188 \\
\hline 178 & 0.0337 & 0.0412 & 0.1765 & 0.4780 & 0.7614 & 4.4454 \\
\hline 179 & 0.8596 & 0.8034 & 0.7434 & 0.3772 & 0.2451 & 7.6021 \\
\hline 180 & 0.3910 & 0.4500 & 0.8571 & 0.1771 & 0.9643 & 8.2858 \\
\hline 181 & 0.3812 & 0.0335 & 0.4742 & 0.9616 & 0.3131 & 4.4204 \\
\hline 182 & 0.0932 & 0.7859 & 0.3164 & 0.6562 & 0.9324 & 7.2789 \\
\hline 183 & 0.6427 & 0.5295 & 0.7836 & 0.1365 & 0.9827 & 8.7062 \\
\hline 184 & 0.8294 & 0.1850 & 0.3612 & 0.0970 & 0.3930 & 4.7227 \\
\hline 185 & 0.3806 & 0.9405 & 0.4711 & 0.7344 & 0.3295 & 6.8443 \\
\hline 186 & 0.9313 & 0.7566 & 0.7370 & 0.2105 & 0.5830 & 8.7730 \\
\hline 187 & 0.3264 & 0.8321 & 0.8396 & 0.4447 & 0.6439 & 8.5421 \\
\hline 188 & 0.4405 & 0.3639 & 0.4695 & 0.4465 & 0.6871 & 5.8276 \\
\hline 189 & 0.6709 & 0.6583 & 0.0355 & 0.6759 & 0.8252 & 6.0206 \\
\hline 190 & 0.4836 & 0.1906 & 0.6240 & 0.6970 & 0.5428 & 5.9906 \\
\hline 191 & 0.1842 & 0.0782 & 0.8574 & 0.1993 & 0.8308 & 7.2687 \\
\hline 192 & 0.3924 & 0.3597 & 0.5523 & 0.7894 & 0.2094 & 4.3832 \\
\hline 193 & 0.0327 & 0.8136 & 0.3620 & 0.9500 & 0.8606 & 7.4826 \\
\hline 194 & 0.2393 & 0.6213 & 0.4343 & 0.2957 & 0.7943 & 6.2922 \\
\hline 195 & 0.9090 & 0.3484 & 0.2768 & 0.9190 & 0.2461 & 4.8296 \\
\hline 196 & 0.8749 & 0.7321 & 0.0916 & 0.9134 & 0.2724 & 5.1198 \\
\hline 197 & 0.5651 & 0.9125 & 0.1096 & 0.3481 & 0.1310 & 4.4704 \\
\hline 198 & 0.3391 & 0.6068 & 0.4231 & 0.9952 & 0.6479 & 6.1816 \\
\hline 199 & 0.3286 & 0.5211 & 0.3222 & 0.3325 & 0.0500 & 2.7058 \\
\hline 200 & 0.8462 & 0.9062 & 0.5152 & 0.5494 & 0.0233 & 6.5594 \\
\hline 201 & 0.0476 & 0.7180 & 0.0509 & 0.4954 & 0.0118 & 2.0701 \\
\hline
\end{tabular}


Table A-1 The Data Used for Developing Neural Network in Section 3.2.1-Cont'd

\begin{tabular}{|c|c|c|c|c|c|c|}
\hline S.N. & $\mathbf{x}_{1}$ & $\mathbf{x}_{2}$ & $\mathbf{x}_{3}$ & $\mathbf{x}_{4}$ & $\mathbf{x}_{5}$ & Actual y \\
\hline 202 & 0.4931 & 0.3284 & 0.1354 & 0.8006 & 0.7770 & 5.1368 \\
\hline 203 & 0.8645 & 0.2591 & 0.6253 & 0.0123 & 0.4648 & 6.0181 \\
\hline 204 & 0.8264 & 0.5410 & 0.4129 & 0.7736 & 0.2639 & 5.4281 \\
\hline 205 & 0.3900 & 0.7750 & 0.9228 & 0.1586 & 0.9456 & 9.5727 \\
\hline 206 & 0.5144 & 0.6677 & 0.0217 & 0.3597 & 0.2637 & 3.1637 \\
\hline 207 & 0.9533 & 0.2478 & 0.8995 & 0.1411 & 0.7346 & 8.7750 \\
\hline 208 & 0.5291 & 0.6454 & 0.3536 & 0.5909 & 0.0597 & 3.7880 \\
\hline 209 & 0.4940 & 0.0885 & 0.4985 & 0.8502 & 0.7788 & 6.5215 \\
\hline 210 & 0.0896 & 0.1014 & 0.1736 & 0.1966 & 0.9558 & 4.9803 \\
\hline 211 & 0.4932 & 0.2320 & 0.4239 & 0.4991 & 0.2663 & 3.9914 \\
\hline 212 & 0.6681 & 0.4161 & 0.4060 & 0.1667 & 0.8554 & 6.5625 \\
\hline 213 & 0.0439 & 0.6841 & 0.1388 & 0.8801 & 0.6208 & 4.9409 \\
\hline 214 & 0.3526 & 0.9460 & 0.8034 & 0.1935 & 0.7740 & 9.5377 \\
\hline 215 & 0.4857 & 0.1074 & 0.0688 & 0.7523 & 0.7981 & 4.8105 \\
\hline 216 & 0.4901 & 0.6029 & 0.0443 & 0.9058 & 0.6762 & 4.9713 \\
\hline 217 & 0.1889 & 0.2873 & 0.5162 & 0.5899 & 0.7016 & 5.7816 \\
\hline 218 & 0.5348 & 0.5360 & 0.9350 & 0.0104 & 0.8186 & 8.1508 \\
\hline 219 & 0.1610 & 0.0418 & 0.8916 & 0.1728 & 0.2197 & 4.9126 \\
\hline 220 & 0.5544 & 0.3034 & 0.1748 & 0.6127 & 0.3957 & 3.7633 \\
\hline 221 & 0.5053 & 0.4064 & 0.7773 & 0.9292 & 0.1089 & 5.2206 \\
\hline 222 & 0.5106 & 0.8627 & 0.8675 & 0.5647 & 0.3349 & 8.0086 \\
\hline 223 & 0.1902 & 0.7687 & 0.2353 & 0.8232 & 0.2655 & 4.3453 \\
\hline 224 & 0.8050 & 0.5386 & 0.5477 & 0.2707 & 0.1147 & 4.9347 \\
\hline 225 & 0.4309 & 0.4760 & 0.8922 & 0.2891 & 0.8811 & 8.3256 \\
\hline 226 & 0.1322 & 0.7747 & 0.5724 & 0.1743 & 0.8014 & 7.3427 \\
\hline 227 & 0.8862 & 0.1516 & 0.8259 & 0.9947 & 0.7942 & 9.0585 \\
\hline 228 & 0.8577 & 0.6988 & 0.8926 & 0.1442 & 0.0134 & 6.4986 \\
\hline 229 & 0.9791 & 0.7953 & 0.6163 & 0.8461 & 0.3546 & 8.2297 \\
\hline 230 & 0.9920 & 0.8411 & 0.4349 & 0.3904 & 0.2068 & 6.9450 \\
\hline 231 & 0.6576 & 0.6502 & 0.7534 & 0.5948 & 0.7933 & 8.6476 \\
\hline 232 & 0.9582 & 0.3646 & 0.2848 & 0.5764 & 0.5002 & 5.8808 \\
\hline 233 & 0.2932 & 0.7870 & 0.2097 & 0.0027 & 0.3066 & 3.7510 \\
\hline 234 & 0.0651 & 0.2372 & 0.9718 & 0.1690 & 0.3072 & 5.5755 \\
\hline 235 & 0.9474 & 0.6717 & 0.6880 & 0.2959 & 0.0241 & 6.0966 \\
\hline
\end{tabular}


Table A-1 The Data Used for Developing Neural Network in Section 3.2.1-Cont'd

\begin{tabular}{|c|c|c|c|c|c|c|}
\hline S.N. & $\mathbf{x}_{\mathbf{1}}$ & $\mathbf{x}_{\mathbf{2}}$ & $\mathbf{x}_{\mathbf{3}}$ & $\mathbf{x}_{\mathbf{4}}$ & $\mathbf{x}_{\mathbf{5}}$ & Actual $\mathbf{y}$ \\
\hline 236 & 0.8553 & 0.6513 & 0.1895 & 0.5220 & 0.8510 & 7.1761 \\
\hline 237 & 0.6433 & 0.9528 & 0.9217 & 0.1119 & 0.3222 & 8.7330 \\
\hline 238 & 0.9234 & 0.6475 & 0.7131 & 0.1951 & 0.8499 & 9.2132 \\
\hline 239 & 0.8055 & 0.8589 & 0.4852 & 0.4797 & 0.3707 & 7.3146 \\
\hline 240 & 0.5252 & 0.2896 & 0.3573 & 0.5916 & 0.3944 & 4.4008 \\
\hline 241 & 0.0918 & 0.8950 & 0.3130 & 0.4616 & 0.9318 & 7.8265 \\
\hline 242 & 0.1734 & 0.6991 & 0.0159 & 0.9045 & 0.5308 & 4.2228 \\
\hline 243 & 0.2934 & 0.1362 & 0.8896 & 0.2738 & 0.8035 & 7.4756 \\
\hline 244 & 0.5374 & 0.9794 & 0.4441 & 0.3150 & 0.4848 & 7.6721 \\
\hline 245 & 0.1378 & 0.6899 & 0.4213 & 0.6860 & 0.9035 & 7.1505 \\
\hline 246 & 0.7730 & 0.3125 & 0.3326 & 0.8647 & 0.5728 & 5.8385 \\
\hline 247 & 0.1304 & 0.3856 & 0.7780 & 0.5945 & 0.0144 & 4.1466 \\
\hline 248 & 0.9500 & 0.5251 & 0.6080 & 0.1306 & 0.8014 & 8.2386 \\
\hline 249 & 0.0328 & 0.5564 & 0.7400 & 0.8584 & 0.9934 & 8.3791 \\
\hline 250 & 0.2637 & 0.4944 & 0.1119 & 0.5518 & 0.0026 & 1.7028 \\
\hline
\end{tabular}


Table A-2 Tree Data Set [47]-

\begin{tabular}{|c|c|c|c|}
\hline Obs. No. & Rootstock & Trunk Girth & Weight \\
\hline 1 & I & 358 & 760 \\
\hline 2 & I & 375 & 821 \\
\hline 3 & I & 393 & 928 \\
\hline 4 & I & 394 & 1009 \\
\hline 5 & I & 360 & 766 \\
\hline 6 & I & 351 & 726 \\
\hline 7 & I & 398 & 1209 \\
\hline 8 & I & 362 & 750 \\
\hline 9 & II & 409 & 1036 \\
\hline 10 & II & 406 & 1094 \\
\hline 11 & II & 487 & 1635 \\
\hline 12 & II & 498 & 1517 \\
\hline 13 & II & 438 & 1197 \\
\hline 14 & II & 465 & 1244 \\
\hline 15 & II & 469 & 1495 \\
\hline 16 & II & 440 & 1026 \\
\hline 17 & III & 376 & 912 \\
\hline 18 & III & 444 & 1398 \\
\hline 19 & III & 438 & 1197 \\
\hline 20 & III & 467 & 1613 \\
\hline 21 & III & 448 & 1475 \\
\hline 22 & III & 478 & 1571 \\
\hline 23 & III & 457 & 1506 \\
\hline 24 & III & 456 & 1458 \\
\hline 25 & IV & 389 & 944 \\
\hline 26 & IV & 405 & 1241 \\
\hline 27 & IV & 405 & 1023 \\
\hline
\end{tabular}


Table A-2 Tree Data Set [47]-Cont'd

\begin{tabular}{|c|c|c|c|}
\hline Obs. No. & Rootstock & Trunk Girth & Weight \\
\hline 28 & IV & 392 & 1067 \\
\hline 29 & IV & 327 & 693 \\
\hline 30 & IV & 395 & 1085 \\
\hline 31 & IV & 427 & 1242 \\
\hline 32 & IV & 385 & 1017 \\
\hline 33 & $\mathrm{~V}$ & 404 & 1084 \\
\hline 34 & $\mathrm{~V}$ & 416 & 1151 \\
\hline 35 & V & 479 & 1381 \\
\hline 36 & $\mathrm{~V}$ & 442 & 1242 \\
\hline 37 & $\mathrm{~V}$ & 347 & 673 \\
\hline 38 & V & 441 & 1137 \\
\hline 39 & $\mathrm{~V}$ & 464 & 1455 \\
\hline 40 & $\mathrm{~V}$ & 457 & 1325 \\
\hline 41 & VI & 376 & 800 \\
\hline 42 & VI & 314 & 606 \\
\hline 43 & VI & 375 & 790 \\
\hline 44 & VI & 399 & 853 \\
\hline 45 & VI & 334 & 610 \\
\hline 46 & VI & 321 & 562 \\
\hline 47 & VI & 363 & 707 \\
\hline 48 & VI & 395 & 952 \\
\hline 49 & VII & 266 & 414 \\
\hline 50 & VII & 241 & 335 \\
\hline 51 & VII & 380 & 885 \\
\hline 52 & VII & 401 & 1012 \\
\hline 53 & VII & 296 & 489 \\
\hline 54 & VII & 315 & 616 \\
\hline 55 & VII & 358 & 788 \\
\hline
\end{tabular}


Table A-2 Tree Data Set [47]-Cont'd

\begin{tabular}{|c|c|c|c|}
\hline Obs. No. & Rootstock & Trunk Girth & Weight \\
\hline 56 & VII & 343 & 733 \\
\hline 57 & IX & 231 & 375 \\
\hline 58 & IX & 250 & 410 \\
\hline 59 & IX & 219 & 335 \\
\hline 60 & IX & 275 & 560 \\
\hline 61 & IX & 205 & 251 \\
\hline 62 & IX & 213 & 272 \\
\hline 63 & IX & 266 & 478 \\
\hline 64 & IX & 226 & 278 \\
\hline 65 & $\mathrm{X}$ & 299 & 506 \\
\hline 66 & $\mathrm{X}$ & 381 & 882 \\
\hline 67 & $\mathrm{X}$ & 362 & 737 \\
\hline 68 & $\mathrm{X}$ & 372 & 772 \\
\hline 69 & $\mathrm{X}$ & 369 & 827 \\
\hline 70 & $\mathrm{X}$ & 368 & 821 \\
\hline 71 & $\mathrm{X}$ & 408 & 1149 \\
\hline 72 & $\mathrm{X}$ & 410 & 1035 \\
\hline 73 & XII & 431 & 1609 \\
\hline 74 & XII & 465 & 1658 \\
\hline 75 & XII & 484 & 1789 \\
\hline 76 & XII & 527 & 2375 \\
\hline 77 & XII & 463 & 1556 \\
\hline 78 & XII & 412 & 1418 \\
\hline 79 & XII & 514 & 2266 \\
\hline 80 & XII & 522 & 2508 \\
\hline 81 & XIII & 387 & 1052 \\
\hline 82 & XIII & 414 & 1167 \\
\hline 83 & XIII & 387 & 981 \\
\hline
\end{tabular}


Table A-2 Tree Data Set [47]-Cont'd

\begin{tabular}{|c|c|c|c|}
\hline Obs. No. & Rootstock & Trunk Girth & Weight \\
\hline 84 & XIII & 390 & 944 \\
\hline 85 & XIII & 327 & 737 \\
\hline 86 & XIII & 424 & 1392 \\
\hline 87 & XIII & 421 & 1326 \\
\hline 88 & XIII & 382 & 1052 \\
\hline 89 & $\mathrm{XV}$ & 448 & 1258 \\
\hline 90 & $\mathrm{XV}$ & 435 & 1304 \\
\hline 91 & $\mathrm{XV}$ & 451 & 1290 \\
\hline 92 & $\mathrm{XV}$ & 450 & 1288 \\
\hline 93 & $\mathrm{XV}$ & 428 & 1176 \\
\hline 94 & XV & 424 & 1177 \\
\hline 95 & $\mathrm{XV}$ & 482 & 1331 \\
\hline 96 & $\mathrm{XV}$ & 469 & 1490 \\
\hline 97 & XVI & 452 & 1499 \\
\hline 98 & XVI & 412 & 1412 \\
\hline 99 & XVI & 425 & 1488 \\
\hline 100 & XVI & 460 & 1751 \\
\hline 101 & XVI & 464 & 1937 \\
\hline 102 & XVI & 457 & 1823 \\
\hline 103 & XVI & 463 & 1838 \\
\hline 104 & XVI & 473 & 1817 \\
\hline
\end{tabular}




\title{
Appendix B VBA Code for the NN-CI Program
}

\author{
Option Compare Database \\ Option Explicit \\ Private Sub cmbCItech_AfterUpdate() \\ Select Case Me.cmbcItech \\ Case 1 ' Non-linear regression \\ Me.txtbootsamples.Visible = False \\ Me.Label5.Visible = False \\ Case 2 ' Bootstrap \\ Me.txtbootsamples.Visible = True \\ Me.Label5.Visible = True \\ Case 3 'MLE \\ Me.txtbootsamples.Visible = False \\ Me.Label5.Visible = False \\ End Select \\ End Sub
}

Private Sub cmdCalculate_Click()

On Error GoTo errorhandler

Dim i As Long, j As Long, $k$ As Long

Dim IParrscaled() As Double, minvalx() As Double, maxvalx() As Double

Dim matcal As New cMathLib

IParrscaled () = pmtsIP() (0) 'Input array

$\operatorname{minvalX}()=\operatorname{pmtsIP}()(1)$

$\operatorname{maxvalX}()=\operatorname{pmtsIP}()(2)$

Dim Oparrscaled() As Double, minvaly() As Double, maxvaly() As Double

OParrscaled ()$=$ pmtsoP() (0) 'Output array

$\operatorname{minvaly}()=\operatorname{pmtsOP}()(1)$

maxvaly() = pmtsop () (2)

Dim novars As Long, noobs As Long, noitrs As Long, alpha As 
Double, nohn As Byte, CIlevel As Double novars = Forms ("frm_Main"). lstIPFlds.ItemsSelected. Count noobs = Forms ("frm_Main").txtNOB noitrs = Forms ("frm_Main").txtNoIti nohn = Forms ("frm_Main").txtNOHLN alpha = Forms ("frm_Main").txtAlpha CIlevel = Me.cmbCIlevel Dim wts() As Variant, V() As Double, W() As Double, Ypred() As Double, YpredSB() As Double

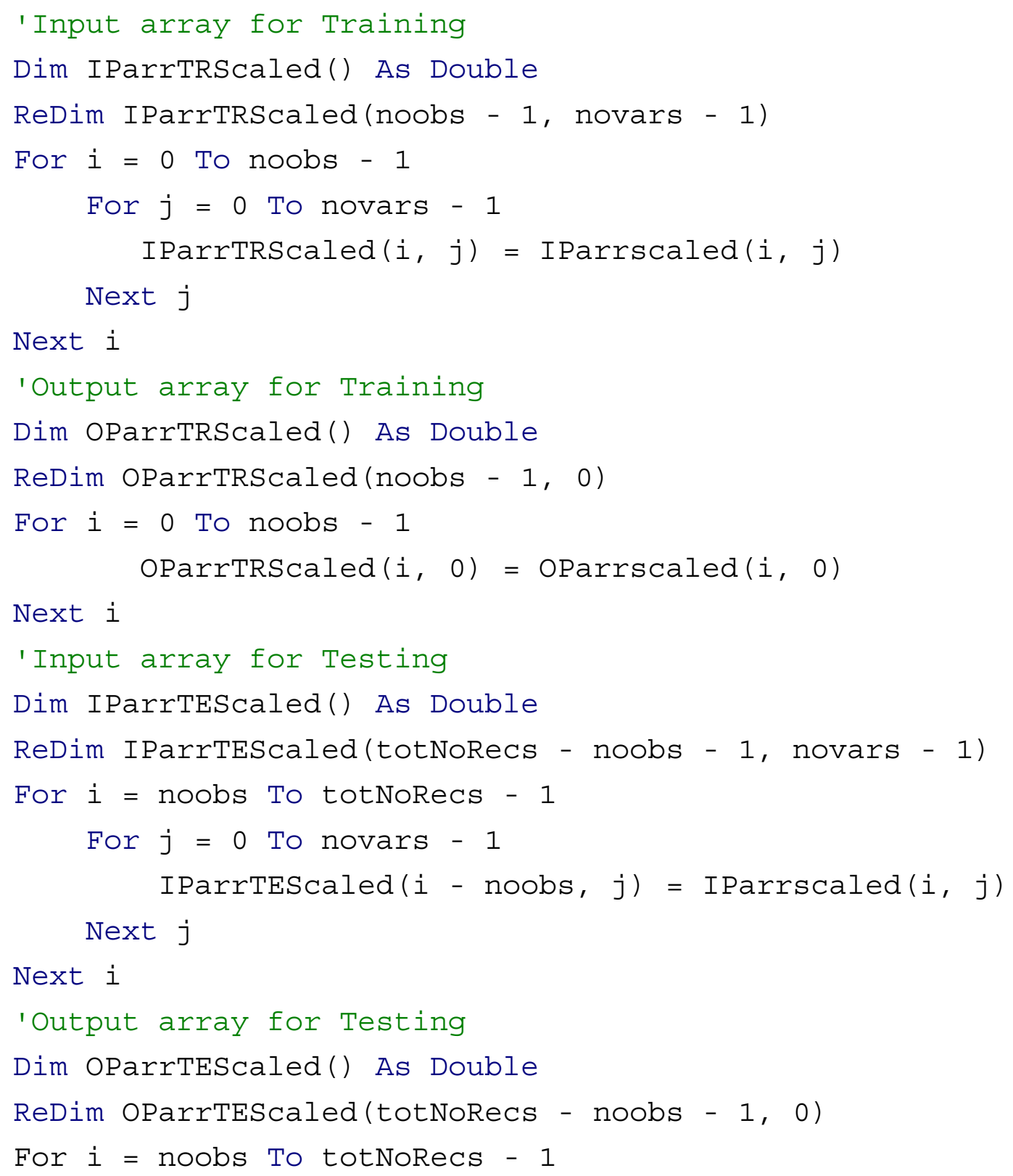


OparrTEScaled $(i-$ noobs, 0$)=$ OParrscaled $(i, 0)$

Next i

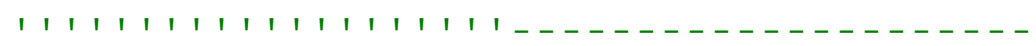

'intFileHandle = FreeFile

'Open wkfolderpath \& ExtractFilename(wkbkpathnname) \& ".txt" For Output As \#intFileHandle

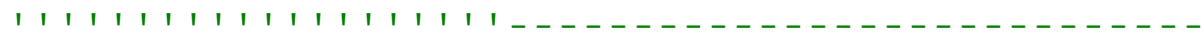

' ' log file

Dim FileNo As Byte

FileNo = FreeFile ' file no for log

open LogFile For Append As \#FileNo

1 1 1 ।

Dim T_dist_Val As Double

Select Case Me.cmbCItech

Case 1 ' Non-linear regression

Call CloseWarning

Dim sigmasQ As Double, sigma As Double, dof_NLR As Long

Dim TotNoParams As Long

TotNoParams $=($ nohn $*($ novars +2$))+1$

For $i=0$ To noobs - 1

'Debug.Print saved_Output(i)

sigmaSQ = sigmaSQ + (saved_Output_SB $(i)$ - Yorg_saved $(i))$

$\wedge 2$

Next i

If noobs $<=$ (nohn * (novars +2$)+1$ ) Then

sigma $=\operatorname{Sqr}($ sigmaSQ)

dof_NLR $=1$

Else

dof_NLR = noobs - TotNoParams

sigma $=\operatorname{Sqr}($ sigmaSQ / dof_NLR)

End If

'Debug.Print sigma

Dim JacobianMatTR() As Double: ReDim JacobianMatTR(noobs - 1 , TotNoParams - 1)

Dim JacobianMatTE_trans() As Double: ReDim

JacobianMatTE_trans (totNoRecs - noobs - 1, TotNoParams - 1) 
Dim JacobianMatAll() As Double: ReDim JacobianMatAll (totNoRecs - 1, TotNoParams - 1)

Dim ZsTR() As Double: ReDim ZsTR (noobs - 1, nohn - 1)

Dim ZsTe() As Double: ReDim ZsTE(totNoRecs - noobs - 1, nohn $-1)$

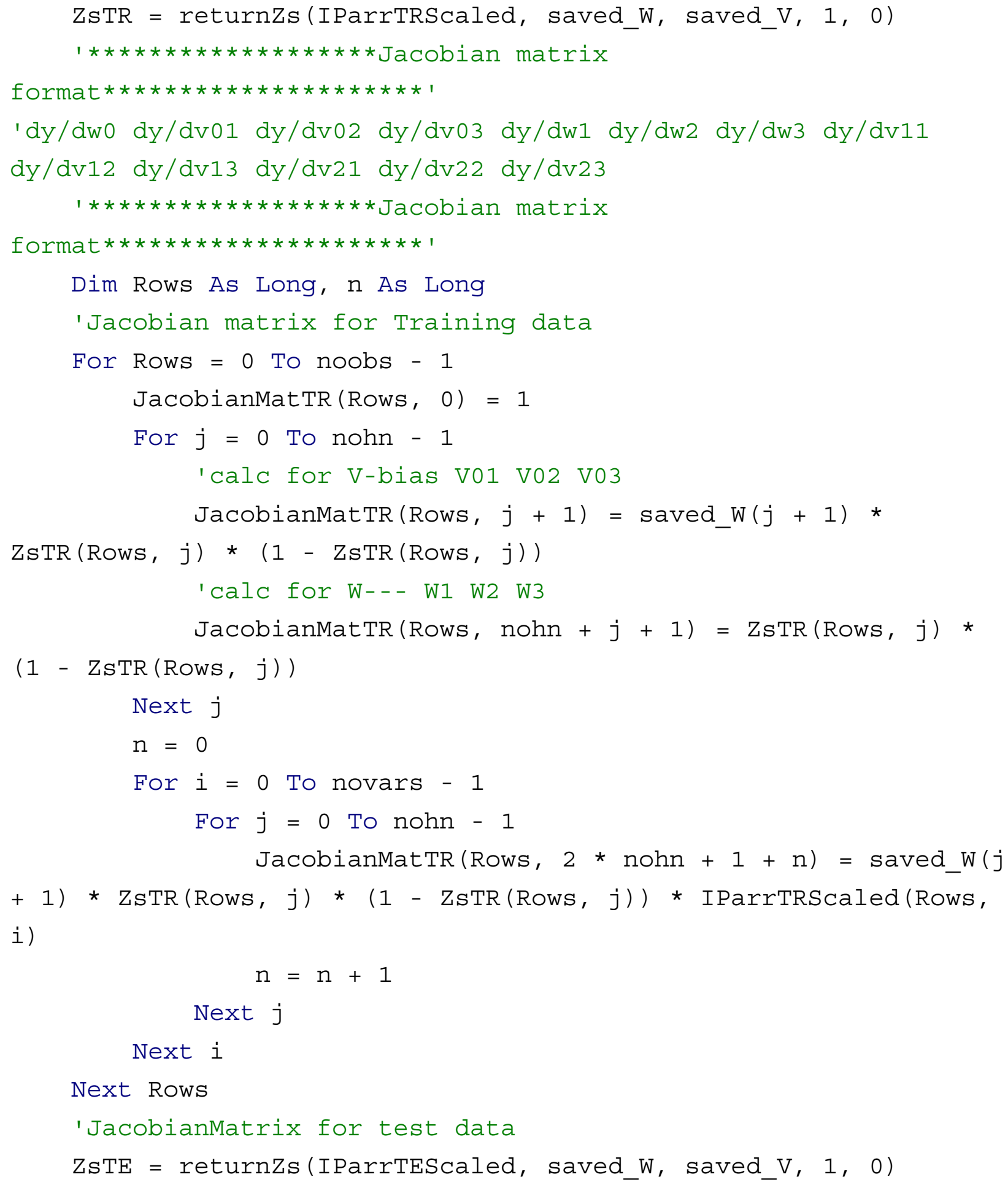




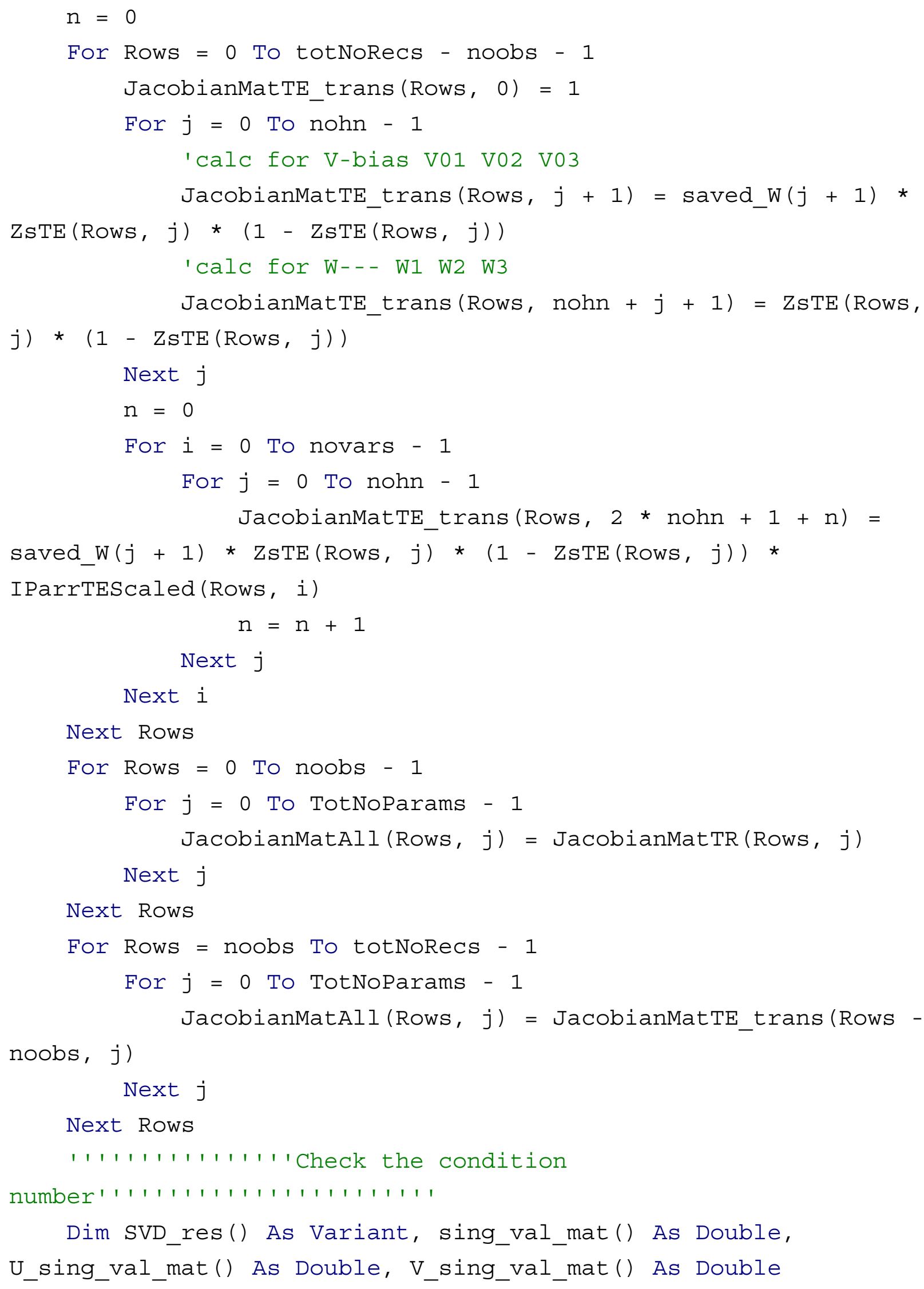

Dim SVD_res() As Variant, sing_val_mat() As Double, U_sing_val_mat() As Double, V_sing_val_mat() As Double 
SVD_res $=$ SVDF (JacobianMatAll) 'finding the SVD for $Z$ or Jacobian Matrix

U_sing_val_mat $=$ SVD_res ()$(0)$
sing_val_mat $=$ SVD_res ()$(1)$
V_sing_val_mat $=$ SVD_res ()$(2)$

Dim CondNo As Double ' condition number

If MinMaxVector(sing_val_mat) $<0$ Then

CondNo = MinMaxVector (sing_val_mat, False) /

MinMaxVector(sing_val_mat)

End If

Dim rhat As Long ' estimated rank of Jacobian matrix

Dim eps As Double, tol As Double

eps $=2^{\wedge}(-52)$ 'Floating-point relative accuracy

tol = MinMaxVector (sing_val_mat, False) * eps

For $i=0$ To UBound (sing_val_mat, 1)

If sing_val_mat(i) > tol Then

rhat $=$ rhat +1

End If

Next i

'note that if CondNo $>10^{\wedge} 8$ and rhat < TotNoParams the CIs are useless

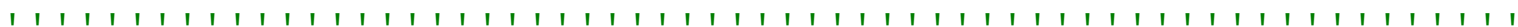

$1 \quad 1 \quad 1 \quad 1 \quad 1$

' Dim JacotransJacoInv() As Double

' Dim SigmaTransSigmaInv() As Double: ReDim

SigmaTransSigmaInv (TotNoParams - 1, TotNoParams - 1)

' If CondNo $>10^{\wedge} 8$ Or rhat < TotNoParams Then

MsgBox "Warning: An error might be generated becauseCondition number of Jacobian matrix $=" \&$ CondNo \& vbCrLf \& _ ' "Rank of Jacobian matrix is = " \& rhat \& " and Total no of parameters are $="$ \& TotNoParams \& vbCrLf, vbInformation, Prog_title

1 End If

$1 * * * * * * * * * * * * * * * * * * * * * \log \operatorname{ing}$ 


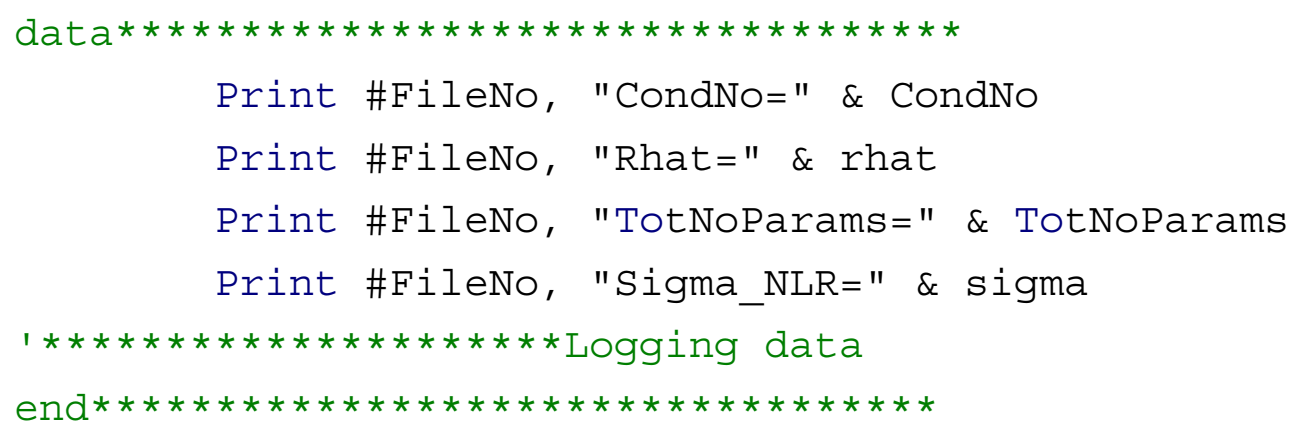

Dim DiagHatmatrix() As Double: ReDim DiagHatmatrix(totNoRecs $-1)$

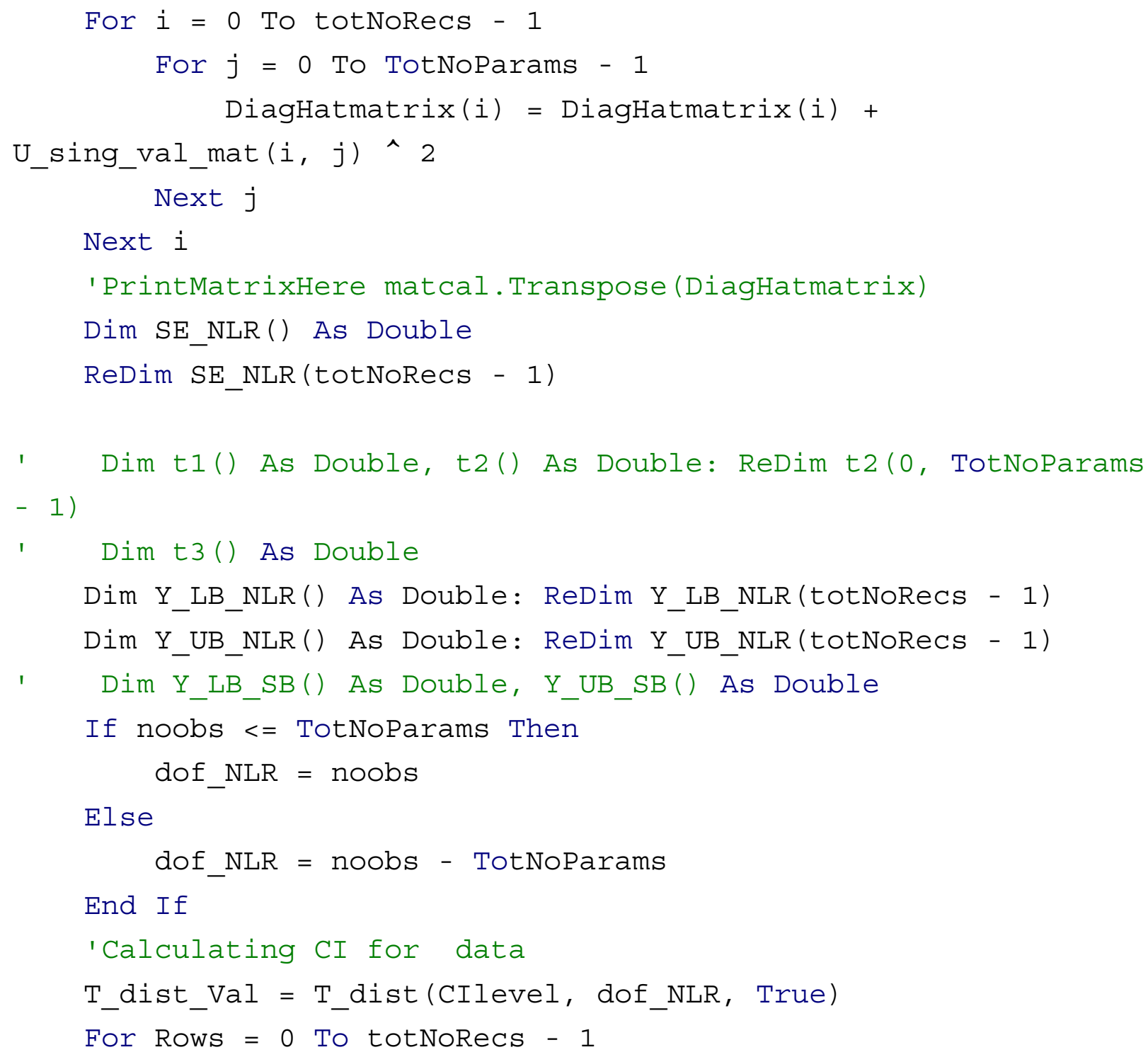




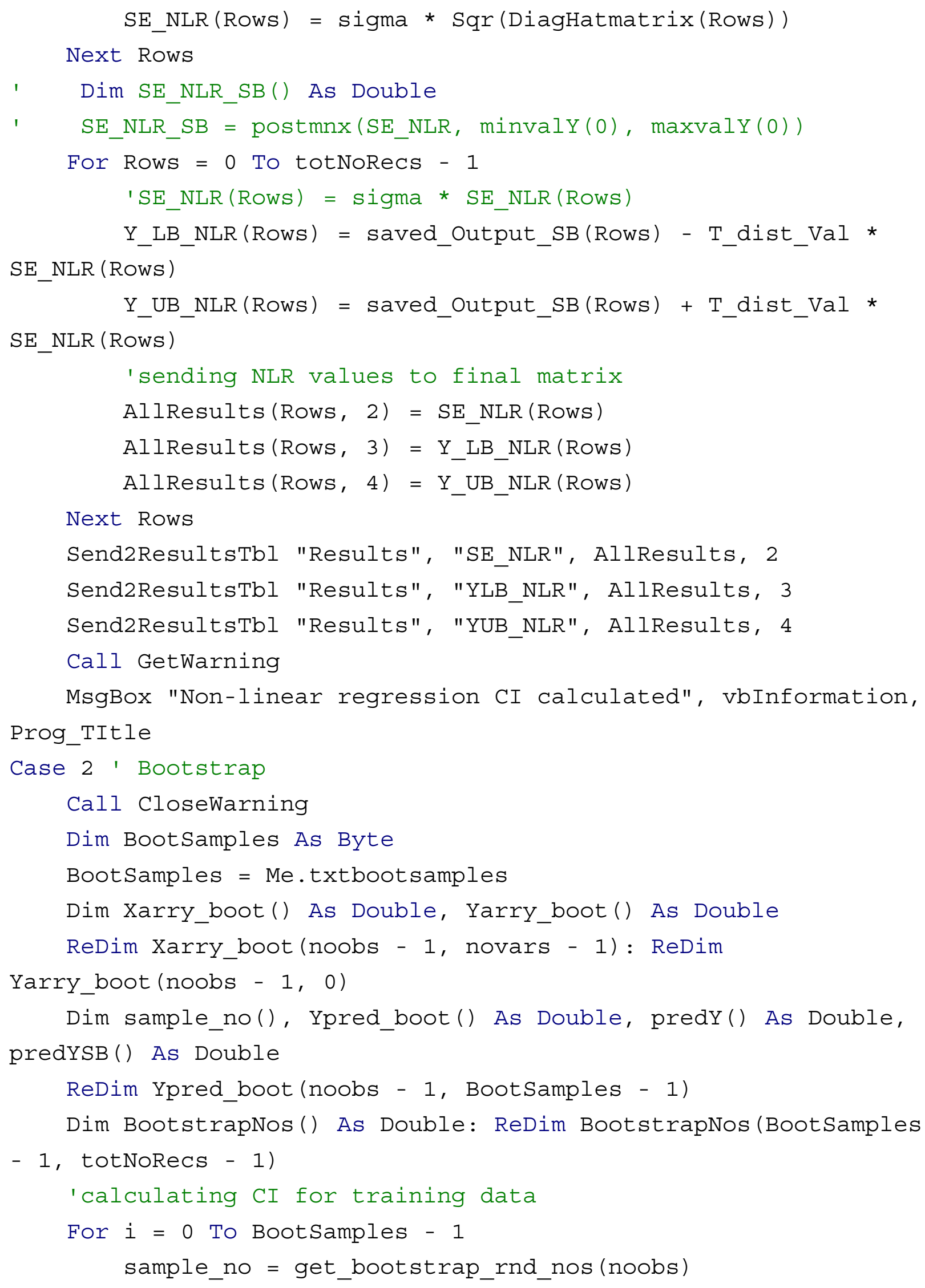




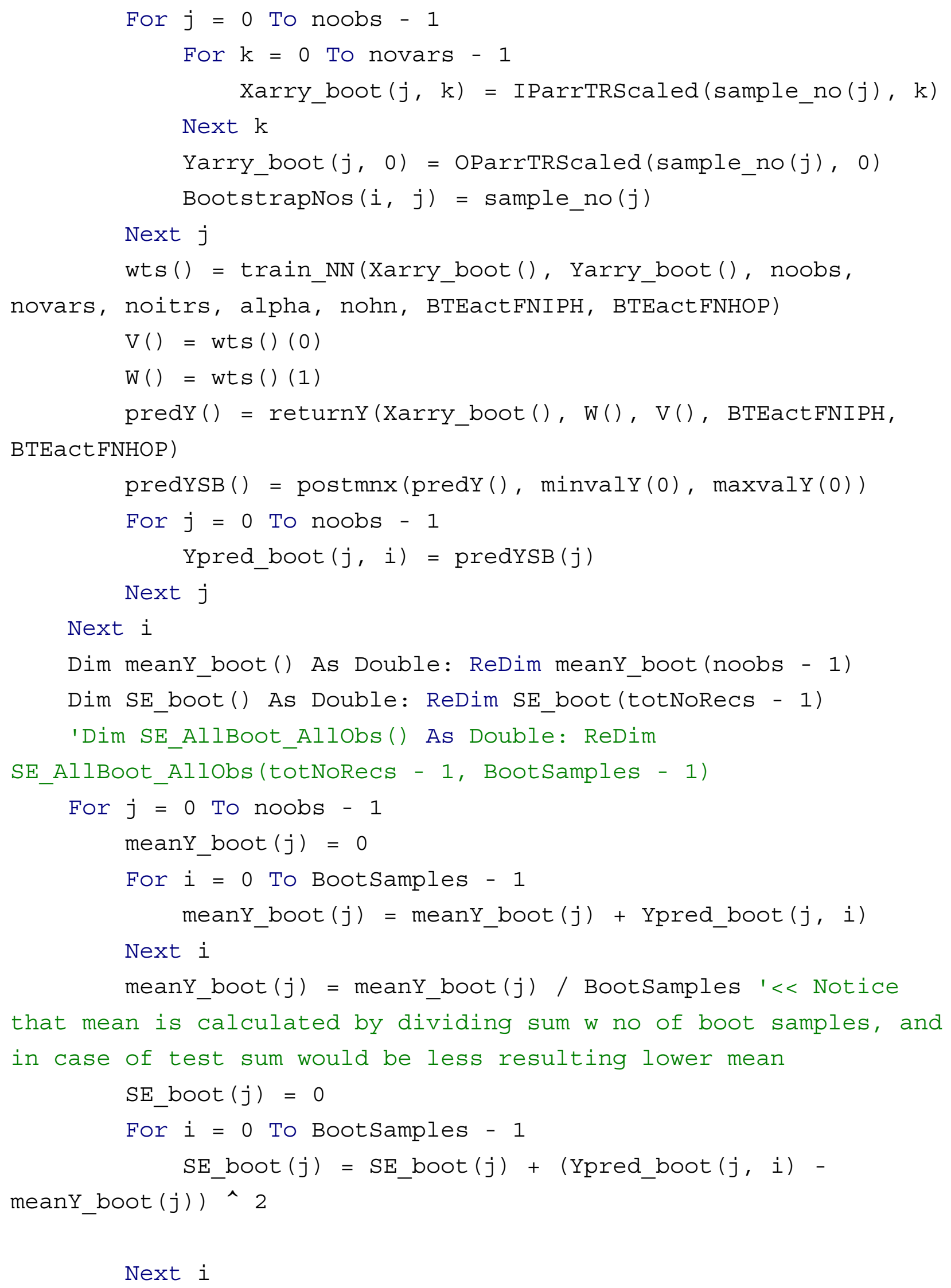




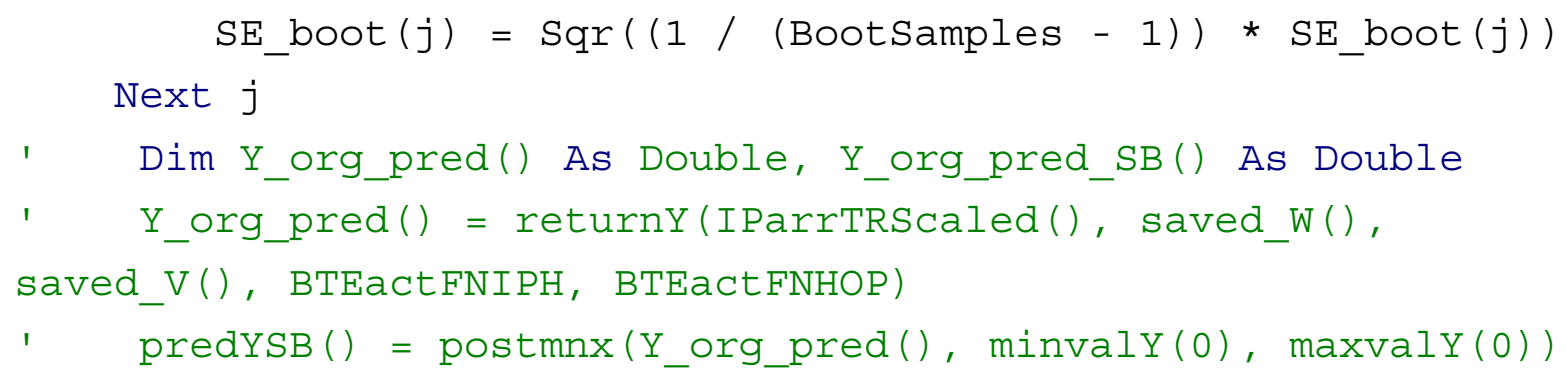

' Code for NN starts

Option Compare Database

Option Explicit

Public TblSelected As Boolean

Public counterwksht As Long

Private Sub cmdCI_Click()

DoCmd.OpenForm "frm_CI_params"

End Sub

Private Sub cmdExport_Click()

counterwksht $=$ counterwksht +1

On Error GoTo errorhandler1

Dim strOPFilename As Variant

Dim obj1 As Object

Dim wb As Object

Dim ws As Object

If fIsAppRunning("Excel") Then

Set obj1 = GetObject(, "Excel.Application")

Else

Set obj1 = CreateObject("Excel.Application")

End If

strOPFilename = obj1.GetSaveAsFilename(, "Excel files (*.xls), *.xls", , "Select the file name/location")

If strOPFilename $=$ "'" Or strOPFilename $=$ False Then 
MsgBox "Not a valid filename, try again", vbCritical, Prog_TItle

GoTo errorhandler1

End If

DoCmd.TransferSpreadsheet acExport, acSpreadsheetTypeExce19, "Results", strOPFilename Set $\mathrm{wb}=$ obj1.Workbooks.Open(strOPFilename)

Set ws = wb.Worksheets("Results")

ws. Name $=$ "sim" \& counterwksht

wb.Save

wb.Close

Set ws $=$ Nothing

Set $\mathrm{wb}=$ Nothing

Set obj $1=$ Nothing

MsgBox "Results exported to " \& strOPFilename \& " succesfully!", vbInformation

Exit Sub 'if there is no error exit the sub

errorhandler1:

If Err.Number $<>0$ Then

Dim msg As String

$\mathrm{msg}=$ "Error \# " \& Str(Err.Number) \& " was generated by "

\& Err.Source \& Chr(13) \& Err.Description

MsgBox msg, vbCritical, Prog_TItle \& "-Error", Err.HelpFile, Err.HelpContext

End If

Set ws $=$ Nothing

Set $\mathrm{wb}=$ Nothing

Set obj $1=$ Nothing

End Sub

Private Sub cmdTest_Click()

Call CloseWarning

Dim IParr() As Variant, strSQL As String, noobs As Long, IPTEarrScaled() As Double noobs $=$ Me.txtNOB

Dim IParrscaled() As Double 
IParrscaled ()$=\operatorname{pmtsIP}()(0)$

Dim IPfldsSelected As Byte

IPfldsSelected $=$ Me.1stIPFlds.ItemsSelected.Count

ReDim IPTEarrScaled(totNoRecs - noobs - 1, IPfldsSelected - 1)

Dim i As Long, j As Long

For $\mathrm{i}=$ noobs To totNoRecs -1

For $\mathrm{j}=0$ To IPfldsSelected -1

IPTEarrScaled $(\mathrm{i}-$ noobs, $\mathrm{j})=\operatorname{IParrscaled}(\mathrm{i}, \mathrm{j})$

Next j

Next i

Dim OParrscaled() As Double, minvalY() As Double, maxvalY() As Double

$\operatorname{minval} \mathrm{Y}()=\operatorname{pmts} \mathrm{OP}()(1)$

$\operatorname{maxvalY}()=\operatorname{pmtsOP}()(2)$

Dim Ypred() As Double

Ypred ()$=$ returnY $($ IPTEarrScaled () , saved_W(), saved_V(), BTEactFNIPH, BTEactFNHOP $)$

Dim YpredSB() As Double

YpredSB ()$=\operatorname{postmnx}($ Ypred, minvalY $(0)$, maxvalY $(0))$

TeMSE $=0$

For $\mathrm{i}=0$ To UBound(Ypred)

TeMSE $=$ TeMSE $+(\text { Yorg_saved }(\mathrm{i}+\text { noobs })-\text { YpredSB }(\mathrm{i}))^{\wedge} 2$

saved_Output $(\mathrm{i}+$ noobs $)=$ Ypred(i)

saved_Output_SB $(\mathrm{i}+$ noobs $)=$ YpredSB $(\mathrm{i})$

AllResults $(i+$ noobs, 1$)=$ YpredSB(i) 'Sending Ypred vals to Allresults matrix

Next i

'***************'"'"'Sending values to results table

Send2ResultsTbl "Results", "Yorg", AllResults, 0

Send2ResultsTbl "Results", "YPred", AllResults, 1

'sending values done

$* * * * * * * * * * * * * * * * * * * * *$ Logging data $* * * * * * * * * * * * * * * * * * * * * * * * * * * * * * * * * *$

Dim FileNo As Byte

FileNo $=$ FreeFile 
Open LogFile For Append As \#FileNo

Print \#FileNo, "TeMSE=" \& TeMSE

Close FileNo

$1 * * * * * * * * * * * * * * * * * * * * * \log \operatorname{lin} g$ data $\operatorname{end} * * * * * * * * * * * * * * * * * * * * * * * * * * * * * * * * * *$

'Call sendvals2XL(wkbkpathnname, YpredSB, noobs +2 , totNoRecs +1 , col2sendData2, wkshtname)

Call GetWarning

MsgBox "Test Complete!", vbInformation, Prog_TItle

If IsFormOpen("frmChart") Then

DoCmd.Close acForm, "frmChart"

DoCmd.OpenForm "frmChart", acNormal

Forms("frmchart").Requery

Else

DoCmd.OpenForm "frmChart", acNormal

Forms("frmchart").Requery

End If

Me.cmdCI.Enabled $=$ True

End Sub

Private Sub cmdTrain_Click()

Call CloseWarning

'If Me.txtwkshtname = "'" Or Me.txtcolnumber = "" Then

' MsgBox "Worksheet name or column number is missing", vbCritical, Prog_TItle

1. Exit Sub

'End If

'wkbkpathnname $=$ Me.txtwkbkpathname

'wkshtname $=$ Me.txtwkshtname

'col2sendData2 = val(Me.txtcolnumber)

inputflds $=$ ""

If Me.lstIPFlds.ItemsSelected.Count $=0$ Then

MsgBox "You have not selected any input field, please select one.", vbCritical, Prog_TItle 


\section{Exit Sub}

End If

If Me.lstOPflds.ItemsSelected.Count $=0$ Then

MsgBox "You have not selected any output field, please select one.", vbCritical, Prog_TItle Exit Sub

End If

Dim inputarr() As Variant, outputarr() As Variant

Dim noobs As Long, noIrs As Long, nohn As Byte, alpha As Double, intsSelected As Long Dim blNW As Boolean 'Nguyen-widrow initilization

intsSelected $=$ Me.1stIPFlds.ItemsSelected.Count

noobs $=$ Me.txtNOB

noIrs $=$ Me.txtNoIti

nohn $=$ Me.txtNOHLN

alpha $=$ Me.txtAlpha

BTEactFNIPH $=$ Me.cmbActFnIPH

BTEactFNHOP $=$ Me.cmdActFnHOP

strSelectedTblName $=$ Me.lstTbls

blNW $=$ Me.cmbNW

Dim cn As New ADODB.Connection

Dim rs As ADODB.Recordset

Set rs $=$ New ADODB.Recordset

Set $\mathrm{cn}=$ CurrentProject.Connection

Dim strSQL As String

inputflds = get_input_flds(Me.lstIPFlds)

strSQL = "SELECT " \& inputflds \& " FROM " \& strSelectedTblName \& ";"

rs.Open strSQL, cn, 1

totNoRecs $=$ rs.RecordCount

ReDim saved_Output(totNoRecs - 1)

ReDim saved_Output_SB(totNoRecs - 1)

ReDim AllResults(totNoRecs - 1, 13)

ReDim Yorg_saved(totNoRecs - 1) 
inputarr $=$ rs.GetRows $($ totNoRecs $)$

Dim inputarrDbl() As Double, IParrscaled() As Double, matcal As New cMathLib

inputarrDbl = mat_transpose_getrows(inputarr())

'Debug.Print NumberOfDimensions(inputarrDbl())

$\operatorname{pmtsIP}()=\operatorname{premnx}($ inputarrDbl $)$

Dim minvalIP_arr() As Double, maxvalIP_arr() As Double, IParrTRScaled() As Double

IParrscaled ()$=\operatorname{pmtsIP}()(0)$ 'Input array

minvalIP_arr ()$=\operatorname{pmtsIP}()(1)$

maxvalIP_arr ()$=\operatorname{pmtsIP}()(2)$

'PrintMatrixHere matcal.Transpose(IParrscaled)

Dim i As Long, j As Long

ReDim IParrTRScaled(noobs - 1, intsSelected - 1)

For $\mathrm{i}=0$ To noobs -1

For $\mathrm{j}=0$ To intsSelected - 1

IParrTRScaled $(\mathrm{i}, \mathrm{j})=\operatorname{IParrscaled}(\mathrm{i}, \mathrm{j})$

Next j

Next i

'For $\mathrm{i}=0$ To UBound(arrB, 1)

' Debug.Print $\operatorname{arrB}(\mathrm{i}) \&$ " " \& $\operatorname{arrC}(\mathrm{i})$

'Next i

rs.Close

OPFld $=$ Me.lstOPflds

strSQL = "SELECT " \& OPFld \& " FROM " \& strSelectedTblName \& ";"

rs.Open strSQL, cn

outputarr $=$ rs.GetRows $($ totNoRecs $)$

Dim OParrscaled() As Double

outputarrDbl = mat_transpose_getrows(outputarr())

'Sending Yorg values to the Final array

For $\mathrm{i}=0$ To totNoRecs -1

AllResults $(\mathrm{i}, 0)=$ outputarrDbl(i, 0)

Yorg_saved(i) = outputarrDbl(i, 0) 
Next i

$\operatorname{pmtsOP}()=\operatorname{premnx}($ outputarrDbl $)$

Dim minvalOP() As Double, maxvalOP() As Double, OParrTRScaled() As Double

OParrscaled ()$=\operatorname{pmtsOP}()(0)$ 'Target Array

$\operatorname{minvalOP}()=\operatorname{pmtsOP}()(1)$

$\operatorname{maxvalOP}()=\operatorname{pmtsOP}()(2)$

'PrintMatrixHere matcal.Transpose(OParrscaled)

ReDim OParrTRScaled(noobs - 1,0)

For $\mathrm{i}=0$ To noobs -1

OParrTRScaled $(\mathrm{i}, 0)=$ OParrscaled $(\mathrm{i}, 0)$

Next i

'For $\mathrm{i}=0$ To UBound(OParrScaled, 1)

'Debug.Print OParrScaled(i)

'Next i

Dim wts() As Variant, V() As Double, W() As Double, Ypred() As Double

wts ()$=$ train_NN(IParrTRScaled(), OParrTRScaled(), noobs, intsSelected, noIrs, alpha, nohn, BTEactFNIPH, BTEactFNHOP, blNW)

$\mathrm{V}()=\mathrm{wts}()(0)$

$\mathrm{W}()=\mathrm{wts}()(1)$

Ypred() = returnY(IParrTRScaled(), W(), V(), BTEactFNIPH, BTEactFNHOP)

Dim YPred_SB() As Double

YPred_SB ()$=\operatorname{postmnx}(\operatorname{Ypred}(), \operatorname{minvalOP}(0), \operatorname{maxvalOP}(0))$

TrMSE $=0$

For $\mathrm{i}=0$ To UBound(Ypred)

TrMSE $=$ TrMSE $+($ Yorg_saved(i) - YPred_SB(i) $) \wedge 2$

saved_Output(i) $=$ Ypred(i)

saved_Output_SB(i)=YPred_SB(i)

AllResults(i, 1) = YPred_SB(i) 'Sending Ypred vals to Allresults matrix

Next i

'Call sendvals2XL(wkbkpathnname, YPred_SB, 2, noobs + 1, col2sendData2, wkshtname) 
$1 * * * * * * * * * * * * * * * * * * * * * \operatorname{Logging} \operatorname{data} * * * * * * * * * * * * * * * * * * * * * * * * * * * * * * * * * *$

LogFile $=$ WkFolderPath \& strSelectedTblName \& ".txt"

Dim FileNo As Byte

FileNo $=$ FreeFile

Open LogFile For Output As \#FileNo

Print \#FileNo, "W"

PrintMatrix matcal.Transpose(W), FileNo

Print \#FileNo, "V"

PrintMatrix matcal.Transpose(V), FileNo

Print \#FileNo, "TrMSE=" \& TrMSE

Close FileNo

$1 * * * * * * * * * * * * * * * * * * * * * \log \operatorname{king}$ data $\operatorname{end} * * * * * * * * * * * * * * * * * * * * * * * * * * * * * * * * * *$

saved_W $=\mathrm{W}$

saved_V $=\mathrm{V}$

rs.Close

Set $\mathrm{rs}=$ Nothing: Set $\mathrm{cn}=$ Nothing

DoCmd.RunSQL "DELETE * FROM Results;" ' Cleaning the results table

Call resetAutoNum("Results", "OBsNo")

Call GetWarning

MsgBox "Training Complete!", vbInformation, Prog_TItle

Me.cmdTest.Enabled $=$ True

End Sub

Private Sub cmdWkShtName_Click()

wkbkpathnname = GetOpenFile("C: Thesis Work", "Select file to output")

Me.txtwkbkpathname $=$ wkbkpathnname

'Dim wkshtnames() As String

'wkshtnames = Getwkshtnames()

'Dim i As Long 
'For $\mathrm{i}=0$ To UBound(wkshtnames, 1)

' Debug.Print wkshtnames(i)

'Next i

End Sub

Private Sub Form_Load()

Dim cnn As New ADODB.Connection

Dim cat As New ADOX.Catalog

Set $\mathrm{cnn}=$ CurrentProject.Connection

Set cat.ActiveConnection $=\mathrm{cnn}$

Dim tbl As ADOX.Table

With Me.lstTbls

For Each tbl In cat.Tables

If tbl.Type $=$ "TABLE" Then

.AddItem Item:=tbl.Name

End If

Next

End With

Set cnn $=$ Nothing

Set cat $=$ Nothing

Me.cmdTest.Enabled $=$ False

Me.cmdTrain. Enabled $=$ False

Me.cmdCI.Enabled $=$ False

End Sub

Private Sub 1stIPFlds_AfterUpdate()

Dim varItem As Variant, ctrl1 As Control

Set ctrl1 $=$ Me.lstIPFlds

Dim intI As Long, intNumColumns As Long

With ctrl1

intNumColumns $=$.ListCount 
If TblSelected Then

For intI $=0$ To intNumColumns -1

Me.1stOPflds.AddItem Item:=.ItemData(intI)

Next intI

TblSelected $=$ False

End If

intNumColumns $=$.ItemsSelected.Count

For Each varItem In .ItemsSelected

If IsValThere(Me.lstOPflds, .ItemData(varItem)) Then

Me.lstOPflds.RemoveItem Index:=.ItemData(varItem)

End If

Next varItem

intNumColumns $=$.ListCount

Dim strval As String

For intI $=0$ To intNumColumns -1

strval $=$.ItemData $($ intI $)$

If Not IsItemSelected(Me.lstIPFlds, strval) And Not IsValThere(Me.lstOPflds, strval) Then Me.lstOPflds.AddItem Item:=.ItemData(intI)

End If

Next intI

End With

End Sub

Private Sub 1stTbls_AfterUpdate()

If Not table_name_exists(Me.lstTbls) Then

MsgBox "Table with name " \& Me.lstTbls \& " does not exists. Please select a table", vbCritical, Prog_TItle

Forms!frm_main!lstTbls.SetFocus

Exit Sub

End If

strSelectedTblName $=$ Me.lstTbls

TblSelected $=$ True 
Me. cmdTrain. Enabled $=$ True

Dim cnn As New ADODB.Connection

Dim rs As ADODB.Recordset

Dim fld As ADODB.Field

Set $\mathrm{cnn}=$ CurrentProject.Connection

Set $\mathrm{rs}=$ New ADODB.Recordset

Me.lstIPFlds.SetFocus

rs.Open strSelectedTblName, cnn, , adCmdTable

Call clear_list(Me.lstIPFlds)

With Me.lstIPFlds

For Each fld In rs.Fields

.AddItem Item:=fld.Name

Next fld

End With

rs.Close

Set cnn $=$ Nothing

Set $\mathrm{rs}=$ Nothing

Set fld $=$ Nothing

Call clear_list(Me.1stOPflds)

End Sub 


\section{Appendix C Simulation Results}

Table C-1 Simulation Results for Case A with Nominal Probability of 95\%

\begin{tabular}{|c|c|c|c|c|c|c|}
\hline \multirow[b]{2}{*}{ 氞 } & \multicolumn{2}{|c|}{ Non-linear Regression } & \multicolumn{2}{|c|}{ Bootstrap } & \multicolumn{2}{|c|}{ MLE } \\
\hline & $\begin{array}{c}\text { Covered } \\
\text { Points }\end{array}$ & $\begin{array}{c}\text { Coverage } \\
\text { Probability } \\
\text { (\%) }\end{array}$ & $\begin{array}{c}\text { Covered } \\
\text { Points }\end{array}$ & $\begin{array}{c}\text { Coverage } \\
\text { Probability } \\
\text { (\%) }\end{array}$ & $\begin{array}{c}\text { Covered } \\
\text { Points }\end{array}$ & $\begin{array}{c}\text { Coverage } \\
\text { Probability } \\
\text { (\%) }\end{array}$ \\
\hline 1 & 239 & 95.60 & 245 & 98.00 & 230 & 92.00 \\
\hline 2 & 239 & 95.60 & 243 & 97.20 & 228 & 91.20 \\
\hline 3 & 240 & 96.00 & 226 & 90.40 & 224 & 89.60 \\
\hline 4 & 242 & 96.80 & 245 & 98.00 & 230 & 92.00 \\
\hline 5 & 238 & 95.20 & 239 & 95.60 & 231 & 92.40 \\
\hline 6 & 241 & 96.40 & 250 & 100.00 & 232 & 92.80 \\
\hline 7 & 239 & 95.60 & 245 & 98.00 & 250 & 100.00 \\
\hline 8 & 239 & 95.60 & 237 & 94.80 & 235 & 94.00 \\
\hline 9 & 240 & 96.00 & 239 & 95.60 & 228 & 91.20 \\
\hline 10 & 239 & 95.60 & 250 & 100.00 & 231 & 92.40 \\
\hline 11 & 238 & 95.20 & 226 & 90.40 & 250 & 100.00 \\
\hline 12 & 235 & 94.00 & 225 & 90.00 & 223 & 89.20 \\
\hline 13 & 239 & 95.60 & 250 & 100.00 & 228 & 91.20 \\
\hline 14 & 239 & 95.60 & 250 & 100.00 & 231 & 92.40 \\
\hline 15 & 239 & 95.60 & 244 & 97.60 & 232 & 92.80 \\
\hline Avg & 239 & 95.63 & 241 & 96.37 & 232 & 92.88 \\
\hline
\end{tabular}


Table C-2 Simulation Results for Case A with Nominal Probability of $\mathbf{9 0 \%}$

\begin{tabular}{|c|c|c|c|c|c|c|}
\hline \multirow[b]{2}{*}{ 总 } & \multicolumn{2}{|c|}{ Non-linear Regression } & \multicolumn{2}{|c|}{ Bootstrap } & \multicolumn{2}{|c|}{ MLE } \\
\hline & $\begin{array}{c}\text { Covered } \\
\text { Points }\end{array}$ & $\begin{array}{c}\text { Coverage } \\
\text { Probability } \\
\text { (\%) }\end{array}$ & $\begin{array}{c}\text { Covered } \\
\text { Points }\end{array}$ & $\begin{array}{c}\text { Coverage } \\
\text { Probability } \\
\text { (\%) }\end{array}$ & $\begin{array}{c}\text { Covered } \\
\text { Points }\end{array}$ & $\begin{array}{c}\text { Coverage } \\
\text { Probability } \\
\text { (\%) }\end{array}$ \\
\hline 1 & 206 & 82.40 & 228 & 91.20 & 193 & 77.20 \\
\hline 2 & 203 & 81.20 & 219 & 87.60 & 191 & 76.40 \\
\hline 3 & 205 & 82.00 & 191 & 76.40 & 188 & 75.20 \\
\hline 4 & 205 & 82.00 & 231 & 92.40 & 192 & 76.80 \\
\hline 5 & 207 & 82.80 & 206 & 82.40 & 197 & 78.80 \\
\hline 6 & 203 & 81.20 & 248 & 99.20 & 193 & 77.20 \\
\hline 7 & 204 & 81.60 & 229 & 91.60 & 250 & 100.00 \\
\hline 8 & 217 & 86.80 & 208 & 83.20 & 207 & 82.80 \\
\hline 9 & 202 & 80.80 & 213 & 85.20 & 189 & 75.60 \\
\hline 10 & 207 & 82.80 & 242 & 96.80 & 194 & 77.60 \\
\hline 11 & 206 & 82.40 & 183 & 73.20 & 250 & 100.00 \\
\hline 12 & 203 & 81.20 & 192 & 76.80 & 186 & 74.40 \\
\hline 13 & 203 & 81.20 & 248 & 99.20 & 193 & 77.20 \\
\hline 14 & 205 & 82.00 & 244 & 97.60 & 192 & 76.80 \\
\hline 15 & 204 & 81.60 & 224 & 89.60 & 191 & 76.40 \\
\hline Avg & 205 & 82.13 & 220 & 88.16 & 200 & 80.16 \\
\hline
\end{tabular}


Table C-3 Simulation Results for Case B with Nominal Probability of $\mathbf{9 5 \%}$

\begin{tabular}{|c|c|c|c|c|c|c|c|}
\hline \multirow[b]{2}{*}{ 离 } & \multirow[b]{2}{*}{$\begin{array}{c}\text { MSE } \\
\text { Training }\end{array}$} & \multicolumn{2}{|c|}{ Non-linear Regression } & \multicolumn{2}{|c|}{ Bootstrap } & \multicolumn{2}{|c|}{ MLE } \\
\hline & & $\begin{array}{c}\text { Covered } \\
\text { Points }\end{array}$ & $\begin{array}{c}\text { Coverage } \\
\text { Probability } \\
\text { (\%) }\end{array}$ & $\begin{array}{c}\text { Covered } \\
\text { Points }\end{array}$ & $\begin{array}{c}\text { Coverage } \\
\text { Probability } \\
\text { (\%) }\end{array}$ & $\begin{array}{c}\text { Covered } \\
\text { Points }\end{array}$ & $\begin{array}{c}\text { Coverage } \\
\text { Probability } \\
(\%)\end{array}$ \\
\hline 1 & 652,950 & 22 & 95.65 & 22 & 95.65 & 22 & 95.65 \\
\hline 2 & 753,655 & 22 & 95.65 & 21 & 91.30 & 19 & 82.61 \\
\hline 3 & 690,692 & 22 & 95.65 & 22 & 95.65 & 21 & 91.30 \\
\hline 4 & 783,757 & 22 & 95.65 & 22 & 95.65 & 22 & 95.65 \\
\hline 5 & 839,712 & 22 & 95.65 & 21 & 91.30 & 22 & 95.65 \\
\hline 6 & 690,745 & 22 & 95.65 & 22 & 95.65 & 20 & 86.96 \\
\hline 7 & 880,970 & 22 & 95.65 & 22 & 95.65 & 21 & 91.30 \\
\hline 8 & 737,897 & 22 & 95.65 & 21 & 91.30 & 21 & 91.30 \\
\hline 9 & 752,819 & 22 & 95.65 & 22 & 95.65 & 20 & 86.96 \\
\hline 10 & 783,204 & 22 & 95.65 & 22 & 95.65 & 20 & 86.96 \\
\hline 11 & 815,248 & 22 & 95.65 & 21 & 91.30 & 21 & 91.30 \\
\hline 12 & 854,376 & 22 & 95.65 & 21 & 91.30 & 21 & 91.30 \\
\hline 13 & 686,712 & 22 & 95.65 & 22 & 95.65 & 22 & 95.65 \\
\hline 14 & 696,833 & 22 & 95.65 & 22 & 95.65 & 21 & 91.30 \\
\hline 15 & 689,696 & 22 & 95.65 & 22 & 95.65 & 20 & 86.96 \\
\hline Avg & 753,951 & 22 & 95.65 & 22 & 94.20 & 21 & 90.72 \\
\hline
\end{tabular}


Table C-4 Simulation Results for Case B with Nominal Probability of $\mathbf{9 0 \%}$

\begin{tabular}{|c|c|c|c|c|c|c|c|}
\hline \multirow[b]{2}{*}{ 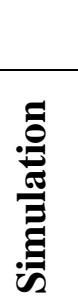 } & \multirow[b]{2}{*}{$\begin{array}{c}\text { MSE } \\
\text { Training }\end{array}$} & \multicolumn{2}{|c|}{ Non-linear Regression } & \multicolumn{2}{|c|}{ Bootstrap } & \multicolumn{2}{|c|}{ MLE } \\
\hline & & $\begin{array}{c}\text { Covered } \\
\text { Points }\end{array}$ & $\begin{array}{c}\text { Coverage } \\
\text { Probability } \\
(\%)\end{array}$ & $\begin{array}{c}\text { Covered } \\
\text { Points }\end{array}$ & $\begin{array}{c}\text { Coverage } \\
\text { Probability } \\
\text { (\%) }\end{array}$ & $\begin{array}{c}\text { Covered } \\
\text { Points }\end{array}$ & $\begin{array}{c}\text { Coverage } \\
\text { Probability } \\
\text { (\%) }\end{array}$ \\
\hline 1 & 652,950 & 21 & 91.30 & 21 & 91.30 & 22 & 95.65 \\
\hline 2 & 753,655 & 22 & 95.65 & 19 & 82.61 & 18 & 78.26 \\
\hline 3 & 690,692 & 21 & 91.30 & 20 & 86.96 & 21 & 91.30 \\
\hline 4 & 783,757 & 22 & 95.65 & 20 & 86.96 & 22 & 95.65 \\
\hline 5 & 839,712 & 21 & 91.30 & 21 & 91.30 & 22 & 95.65 \\
\hline 6 & 690,745 & 21 & 91.30 & 21 & 91.30 & 18 & 78.26 \\
\hline 7 & 880,970 & 21 & 91.30 & 21 & 91.30 & 17 & 73.91 \\
\hline 8 & 737,897 & 21 & 91.30 & 21 & 91.30 & 19 & 82.61 \\
\hline 9 & 752,819 & 21 & 91.30 & 21 & 91.30 & 18 & 78.26 \\
\hline 10 & 783,204 & 21 & 91.30 & 21 & 91.30 & 19 & 82.61 \\
\hline 11 & 815,248 & 21 & 91.30 & 21 & 91.30 & 19 & 82.61 \\
\hline 12 & 854,376 & 21 & 91.30 & 21 & 91.30 & 19 & 82.61 \\
\hline 13 & 686,712 & 21 & 91.30 & 21 & 91.30 & 22 & 95.65 \\
\hline 14 & 696,833 & 21 & 91.30 & 20 & 86.96 & 18 & 78.26 \\
\hline 15 & 689,696 & 22 & 95.65 & 22 & 95.65 & 18 & 78.26 \\
\hline Avg & 753,951 & 21 & 92.17 & 21 & 90.14 & 19 & 84.64 \\
\hline
\end{tabular}


Table C-5 Simulation Results for Case C with Nominal Probability of 95\%

\begin{tabular}{|c|c|c|c|c|c|c|c|}
\hline \multirow[b]{2}{*}{ 总 } & \multirow[b]{2}{*}{$\begin{array}{c}\text { MSE } \\
\text { Training }\end{array}$} & \multicolumn{2}{|c|}{ Non-linear Regression } & \multicolumn{2}{|c|}{ Bootstrap } & \multicolumn{2}{|c|}{ MLE } \\
\hline & & $\begin{array}{c}\text { Covered } \\
\text { Points }\end{array}$ & $\begin{array}{c}\text { Coverage } \\
\text { Probability } \\
(\%)\end{array}$ & $\begin{array}{c}\text { Covered } \\
\text { Points }\end{array}$ & $\begin{array}{c}\text { Coverage } \\
\text { Probability } \\
(\%)\end{array}$ & $\begin{array}{c}\text { Covered } \\
\text { Points }\end{array}$ & $\begin{array}{c}\text { Coverage } \\
\text { Probability } \\
\text { (\%) }\end{array}$ \\
\hline 1 & 0.01148 & 1807 & 90 & 1933 & 97 & 2000 & 100 \\
\hline 2 & 0.01384 & 1797 & 90 & 1983 & 99 & 2000 & 100 \\
\hline 3 & 0.01397 & 1795 & 90 & 1963 & 98 & 2000 & 100 \\
\hline 4 & 0.01223 & 1804 & 90 & 2000 & 100 & 2000 & 100 \\
\hline 5 & 0.01350 & 1802 & 90 & 1999 & 100 & 2000 & 100 \\
\hline 6 & 0.01140 & 1806 & 90 & 1987 & 99 & 2000 & 100 \\
\hline 7 & 0.02401 & 1796 & 90 & 1877 & 94 & 2000 & 100 \\
\hline 8 & 0.01170 & 1802 & 90 & 2000 & 100 & 2000 & 100 \\
\hline 9 & 0.01221 & 1801 & 90 & 1915 & 96 & 2000 & 100 \\
\hline 10 & 0.01317 & 1799 & 90 & 1995 & 100 & 2000 & 100 \\
\hline 11 & 0.01131 & 1802 & 90 & 1997 & 100 & 2000 & 100 \\
\hline 12 & 0.02711 & 1797 & 90 & 1974 & 99 & 2000 & 100 \\
\hline 13 & 0.01254 & 1797 & 90 & 1998 & 100 & 2000 & 100 \\
\hline 14 & 0.01263 & 1797 & 90 & 1978 & 99 & 1795 & 90 \\
\hline 15 & 0.01201 & 1803 & 90 & 1994 & 100 & 2000 & 100 \\
\hline Avg & 0.01421 & 1800 & 90 & 1973 & 99 & 1986 & 99 \\
\hline
\end{tabular}


Table C-6 Simulation Results for Case C with Nominal Probability of $90 \%$

\begin{tabular}{|c|c|c|c|c|c|c|c|}
\hline \multirow[b]{2}{*}{ 氞 } & \multirow[b]{2}{*}{$\begin{array}{c}\text { MSE } \\
\text { Training }\end{array}$} & \multicolumn{2}{|c|}{ Non-linear Regression } & \multicolumn{2}{|c|}{ Bootstrap } & \multicolumn{2}{|c|}{ MLE } \\
\hline & & $\begin{array}{c}\text { Covered } \\
\text { Points }\end{array}$ & $\begin{array}{c}\text { Coverage } \\
\text { Probability } \\
\text { (\%) }\end{array}$ & $\begin{array}{c}\text { Covered } \\
\text { Points }\end{array}$ & $\begin{array}{c}\text { Coverage } \\
\text { Probability } \\
(\%)\end{array}$ & $\begin{array}{c}\text { Covered } \\
\text { Points }\end{array}$ & $\begin{array}{c}\text { Coverage } \\
\text { Probability } \\
\text { (\%) }\end{array}$ \\
\hline 1 & 0.01148 & 1732 & 87 & 1886 & 94 & 2000 & 100 \\
\hline 2 & 0.01384 & 1737 & 87 & 1946 & 97 & 2000 & 100 \\
\hline 3 & 0.01397 & 1738 & 87 & 1925 & 96 & 2000 & 100 \\
\hline 4 & 0.01223 & 1729 & 86 & 1998 & 100 & 2000 & 100 \\
\hline 5 & 0.01350 & 1741 & 87 & 1993 & 100 & 2000 & 100 \\
\hline 6 & 0.01140 & 1733 & 87 & 1950 & 98 & 2000 & 100 \\
\hline 7 & 0.02401 & 1724 & 86 & 1821 & 91 & 2000 & 100 \\
\hline 8 & 0.01170 & 1731 & 87 & 1995 & 100 & 2000 & 100 \\
\hline 9 & 0.01221 & 1732 & 87 & 1857 & 93 & 2000 & 100 \\
\hline 10 & 0.01317 & 1737 & 87 & 1957 & 98 & 2000 & 100 \\
\hline 11 & 0.01131 & 1734 & 87 & 1983 & 99 & 2000 & 100 \\
\hline 12 & 0.02711 & 1720 & 86 & 1932 & 97 & 2000 & 100 \\
\hline 13 & 0.01254 & 1736 & 87 & 1982 & 99 & 2000 & 100 \\
\hline 14 & 0.01263 & 1734 & 87 & 1943 & 97 & 1734 & 87 \\
\hline 15 & 0.01201 & 1728 & 86 & 1960 & 98 & 2000 & 100 \\
\hline Avg & 0.01421 & 1732 & 87 & 1942 & 97 & 1982 & 99 \\
\hline
\end{tabular}


Table C-7 Simulation Results for Case D with Nominal Probability of $\mathbf{9 5 \%}$

\begin{tabular}{|c|c|c|c|c|c|c|c|}
\hline \multirow[b]{2}{*}{ 总 } & \multirow[b]{2}{*}{$\begin{array}{c}\text { MSE } \\
\text { Training }\end{array}$} & \multicolumn{2}{|c|}{ Non-linear Regression } & \multicolumn{2}{|c|}{ Bootstrap } & \multicolumn{2}{|c|}{ MLE } \\
\hline & & $\begin{array}{c}\text { Covered } \\
\text { Points }\end{array}$ & $\begin{array}{c}\text { Coverage } \\
\text { Probability } \\
(\%)\end{array}$ & $\begin{array}{c}\text { Covered } \\
\text { Points }\end{array}$ & $\begin{array}{c}\text { Coverage } \\
\text { Probability } \\
\text { (\%) }\end{array}$ & $\begin{array}{c}\text { Covered } \\
\text { Points }\end{array}$ & $\begin{array}{c}\text { Coverage } \\
\text { Probability } \\
\text { (\%) }\end{array}$ \\
\hline 1 & 17,013 & 90 & 87 & 92 & 88 & 91 & 88 \\
\hline 2 & 19,449 & 89 & 86 & 104 & 100 & 87 & 84 \\
\hline 3 & 21,885 & 91 & 88 & 103 & 99 & 104 & 100 \\
\hline 4 & 20,484 & 91 & 88 & 104 & 100 & 104 & 100 \\
\hline 5 & 17,002 & 89 & 86 & 104 & 100 & 104 & 100 \\
\hline 6 & 17,118 & 89 & 86 & 104 & 100 & 90 & 87 \\
\hline 7 & 19,964 & 89 & 86 & 104 & 100 & 104 & 100 \\
\hline 8 & 17,789 & 90 & 87 & 94 & 90 & 104 & 100 \\
\hline 9 & 17,759 & 90 & 87 & 104 & 100 & 87 & 84 \\
\hline 10 & 17,827 & 89 & 86 & 104 & 100 & 104 & 100 \\
\hline 11 & 17,053 & 91 & 88 & 104 & 100 & 104 & 100 \\
\hline 12 & 26,580 & 95 & 91 & 100 & 96 & 92 & 88 \\
\hline 13 & 19,992 & 89 & 86 & 99 & 95 & 104 & 100 \\
\hline 14 & 22,350 & 92 & 88 & 104 & 100 & 90 & 87 \\
\hline 15 & 17,405 & 89 & 86 & 100 & 96 & 90 & 87 \\
\hline Avg & 19,311 & 90 & 87 & 102 & 98 & 97 & 94 \\
\hline
\end{tabular}


Table C-8 Simulation Results for Case D with Nominal Probability of $\mathbf{9 0 \%}$

\begin{tabular}{|c|c|c|c|c|c|c|c|}
\hline \multirow[b]{2}{*}{ 总 } & \multirow[b]{2}{*}{$\begin{array}{c}\text { MSE } \\
\text { Training }\end{array}$} & \multicolumn{2}{|c|}{ Non-linear Regression } & \multicolumn{2}{|c|}{ Bootstrap } & \multicolumn{2}{|c|}{ MLE } \\
\hline & & $\begin{array}{c}\text { Covered } \\
\text { Points }\end{array}$ & $\begin{array}{c}\text { Coverage } \\
\text { Probability } \\
(\%)\end{array}$ & $\begin{array}{c}\text { Covered } \\
\text { Points }\end{array}$ & $\begin{array}{c}\text { Coverage } \\
\text { Probability } \\
\text { (\%) }\end{array}$ & $\begin{array}{c}\text { Covered } \\
\text { Points }\end{array}$ & $\begin{array}{c}\text { Coverage } \\
\text { Probability } \\
\text { (\%) }\end{array}$ \\
\hline 1 & 17,013 & 85 & 82 & 89 & 86 & 85 & 82 \\
\hline 2 & 19,449 & 85 & 82 & 99 & 95 & 85 & 82 \\
\hline 3 & 21,885 & 85 & 82 & 96 & 92 & 104 & 100 \\
\hline 4 & 20,484 & 85 & 82 & 104 & 100 & 104 & 100 \\
\hline 5 & 17,002 & 85 & 82 & 96 & 92 & 104 & 100 \\
\hline 6 & 17,118 & 85 & 82 & 99 & 95 & 85 & 82 \\
\hline 7 & 19,964 & 85 & 82 & 102 & 98 & 104 & 100 \\
\hline 8 & 17,789 & 85 & 82 & 87 & 84 & 104 & 100 \\
\hline 9 & 17,759 & 85 & 82 & 103 & 99 & 83 & 80 \\
\hline 10 & 17,827 & 85 & 82 & 99 & 95 & 104 & 100 \\
\hline 11 & 17,053 & 85 & 82 & 97 & 93 & 104 & 100 \\
\hline 12 & 26,580 & 87 & 84 & 97 & 93 & 84 & 81 \\
\hline 13 & 19,992 & 85 & 82 & 91 & 88 & 104 & 100 \\
\hline 14 & 22,350 & 85 & 82 & 102 & 98 & 81 & 78 \\
\hline 15 & 17,405 & 85 & 82 & 92 & 88 & 84 & 81 \\
\hline Avg & 19,311 & 85 & 82 & 97 & 93 & 95 & 91 \\
\hline
\end{tabular}




\section{Appendix D Gradient or First Order Derivatives Calculation Code}

deltatmp $=1$

For Rows $=0$ To totNoRecs -1

For $\mathrm{j}=0$ To nohn -1

GradientW $(\mathrm{j}+1)=\operatorname{AllZs}($ Rows, $\mathrm{j}) *$ deltatmp

$\operatorname{DelHid}(\mathrm{j})=$ saved_W $(\mathrm{j}+1) *$ deltatmp

$\operatorname{DelHid}(\mathrm{j})=\operatorname{DelHid}(\mathrm{j}) * \operatorname{actfunction\_ der}($ AllZs(Rows, j), BTEactFNIPH)

Next $\mathrm{j}$

GradientW $(0)=1$

For $\mathrm{j}=0$ To nohn -1

For $\mathrm{i}=0$ To novars -1

GradientV(i, j) = IParrFull(Rows, i) * DelHid(j)

Next i

GradientVb(j) = DelHid(j)

Next j

tmpXX $=$ Put_grads_vector(GradientW, GradientV, GradientVb, novars, TotNoParams, nohn)

'PrintMatrixHere tmpXX

'********************Jacobian matrix format $* * * * * * * * * * * * * * * * * * * * * 1$

'dy/dw0 dy/dw1 dy/dw2 dy/dw3 dy/dv01 dy/dv02 dy/dv03 dy/dv11 dy/dv12 dy/dv13 dy/dv21 $\mathrm{dy} / \mathrm{dv} 22 \mathrm{dy} / \mathrm{dv} 23$

For $\mathrm{i}=0$ To TotNoParams -1

JacobianMatAll(Rows, i) $=\operatorname{tmpXX(i)~}$

Next i

Next Rows 


\section{Appendix E Program Manual}

There are various input boxes and drop-down lists where the neural network parameters are specified and the data table is selected. The inputs to the program are (see Figure E-1):

1. Number of observations used for training (remaining observations of the data set will be used for testing)

2. Number of hidden layer neurons

3. Number of iterations

4. Learning rate

5. Usage of Nguyen-Widrow random weight initialization,

6. Activation function for the hidden layer

7. Activation function for the output layer

8. Data table

9. Input fields (variables)

10. Output field (variable)

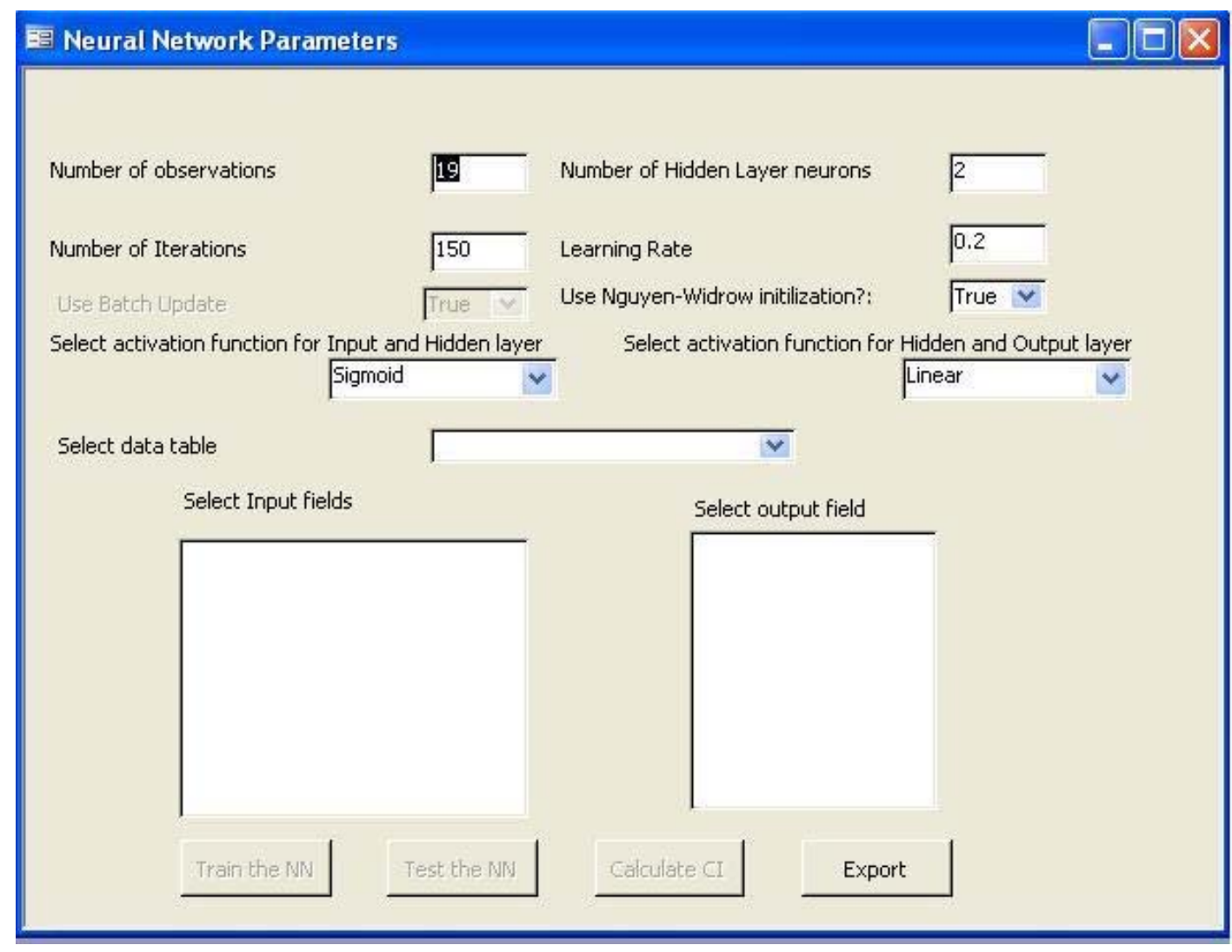

Figure E-1 Main Screen of the Program 
The possible values of these inputs are given in Table E-1. It should be noted that these possible values are just recommendations or tested values with this program. Experiments can be done using different values with the inputs- number of iterations, number of hidden layer neurons, learning rate, and activation functions.

Table E-1 Inputs and the Possible Values to the Program

\begin{tabular}{|c|c|c|}
\hline Input & Possible Values & Data Type \\
\hline $\begin{array}{l}\text { Number of observations used for } \\
\text { training }\end{array}$ & $\begin{array}{l}\text { About } 80-90 \% \text { of the total number of observations in } \\
\text { the data set }\end{array}$ & Integer \\
\hline Number of hidden layer neurons & From 1 to 15 based on the complexity of the data & Integer \\
\hline Number of iterations & $>10$ & Integer \\
\hline Learning rate & Between 0.1 and 0.9 & Double \\
\hline $\begin{array}{l}\text { Usage of Nguyen-Widrow random } \\
\text { weight initialization }\end{array}$ & True/False & Boolean \\
\hline Activation function for the hidden layer & Linear/Sigmoid/Bi-polar Sigmoid/Tanh & Text \\
\hline Activation function for the output layer & Linear/Sigmoid/Bi-polar Sigmoid/Tanh & Text \\
\hline Data table & Different data tables in the database & Text \\
\hline Input fields (variables) and & Fields in the data table & Text \\
\hline Output field (variable) & Fields in the data table & Text \\
\hline
\end{tabular}

After the input fields are selected, the button 'Train the NN' can be used. Once the training is complete, a message box with the information that the training is complete will pop up. After completion of training, "Test the NN" button can be used. Testing should be fast compared to the training and after completion of testing, a message box with the information that "Test is complete" will pop up. Clicking "OK" on this message box will generate two plots of actual data vs. the output of the NN as shown in Figure E-2. The plot on the left side is a scatter plot between actual data and the output of the NN. The plot on the right side is a line plot with two different series of actual data points and the output of the NN. This window will also show Mean Squared Error (MSE) for the training and the test data set.

Once the training and the testing of the NN is complete, confidence intervals can be computed using the "Calculate Cl" button. Clicking this button will bring one more window, where the confidence interval estimation technique and the confidence level should be selected. This screen is shown in Figure E-3. 


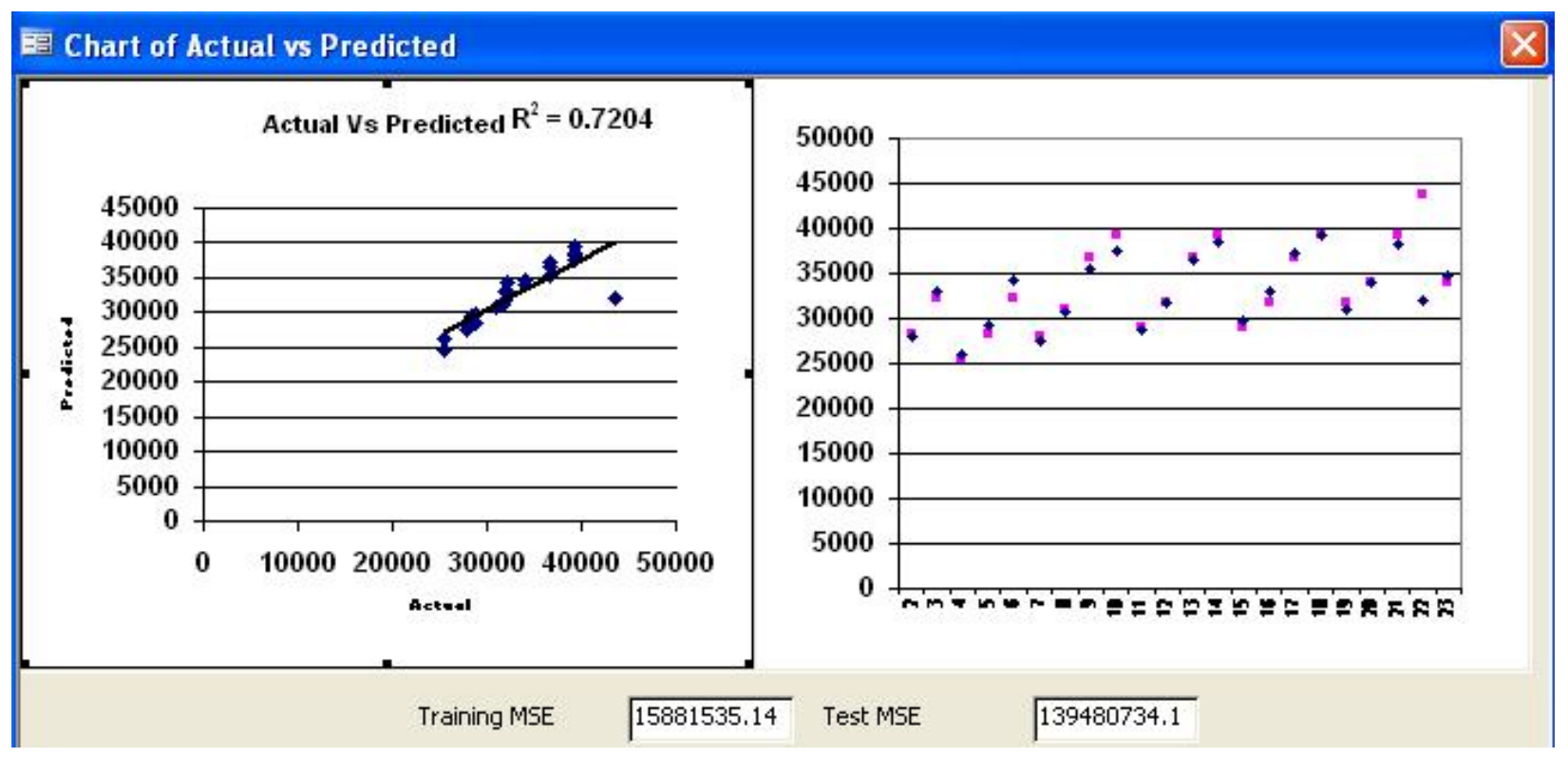

Figure E-2 Plots Generated by the Program

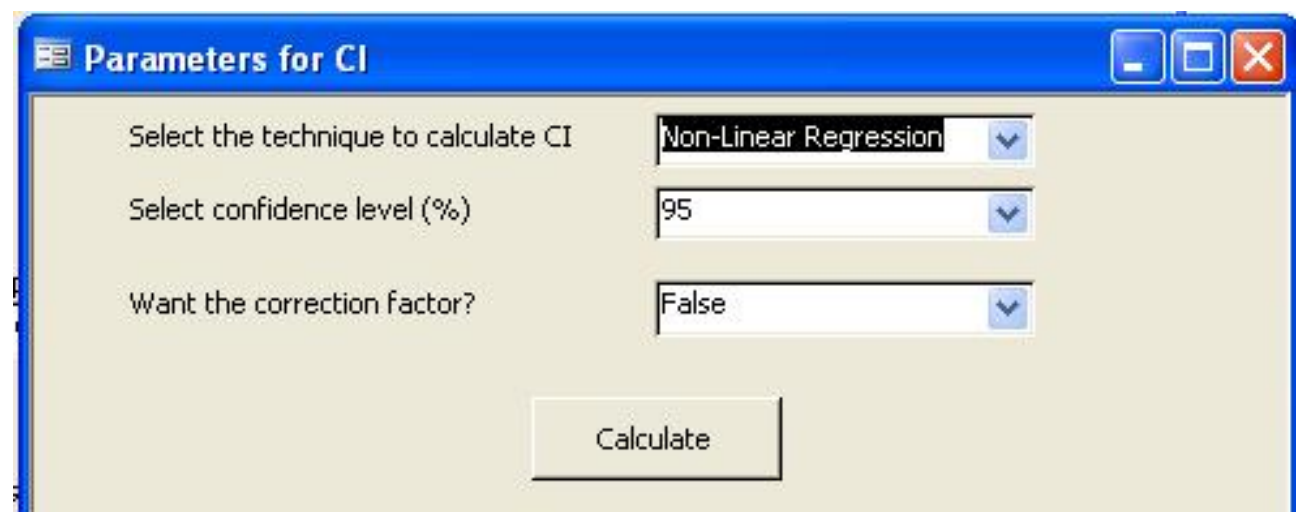

Figure E-3 Confidence Intervals Parameters Form

For the non-linear regression technique, there is an option of using a correction factor (by default, this value is set to false). If bootstrapping as a technique is selected, then there is an option of entering the number of bootstrapping samples. Generally, this value should be greater than 20. After calculation of the intervals, a message box pops up and the results should be saved in the "Results" table. This program will create a text file with the name of the data table selected in the same folder where this file is kept. This text file will have the final weights, training and test MSE, and the condition number and rhat for the non-linear regression technique. 


\section{Appendix F T-test for the Means of NLR and Bootstrap}

T-tests were conducted to test if the average $\mid$ CP-90| and $\mid$ CP-95| obtained by NLR and bootstrap were equal for critical alpha levels of 0.05 and 0.1 . The tests conducted are shown in Figure F-1 [48]. The test results for $\alpha=0.05$ and $\alpha=0.1$ are shown in Table F-1 and Table F-2 respectively.

$H_{0}: \mu_{N L R}=\mu_{B o o t}$
$H_{a}: \mu_{N L R} \neq \mu_{B o o t}$
Test-Statistic: $\mathrm{T}=\frac{\bar{Y}_{N L R}-\bar{Y}_{B o o t}}{\sqrt{s_{N L R}^{2} / N_{N L R}+s_{B o o t}^{2} / N_{B o o t}}}$
where: $\bar{Y}_{N L R}$ and $\bar{Y}_{B o o t}$ are the sample means and $s_{N L R}^{2}$ and $s_{B o o t}^{2}$ are the variances
Significance Level: $\alpha$
Conclusion: If $|\mathrm{T}| \leq t(\alpha, v)$, conclude $H_{0}$, otherwise conclude $H_{a}$
where: $v=\frac{\left(s_{N L R}^{2} / N_{N L R}+s_{B o o t}^{2} / N_{B o o t}\right)^{2}}{\left(s_{N L R}^{2} / N_{N L R}\right)^{2} /\left(N_{N L R}-1\right)+\left(s_{B o o t}^{2} / N_{B o o t}\right)^{2} /\left(N_{B o o t}-1\right)}$

Figure F-1 T-test for Comparing the Means

Table F-1 T-tests Results for the Averages of |CP-95|

\begin{tabular}{|c|c|c|c|c|c|c|c|c|c|c|}
\hline & \multicolumn{2}{|c|}{ NLR } & \multicolumn{2}{|c|}{ Boot } & \multirow{2}{*}{$\begin{array}{l}\text { T- } \\
\text { stat }\end{array}$} & \multirow{2}{*}{ dof } & \multirow{2}{*}{$\begin{array}{l}\text { T-critical } \\
\alpha=0.05\end{array}$} & \multirow{2}{*}{$\begin{array}{c}\text { T-critical } \\
\alpha=0.1\end{array}$} & \multirow{2}{*}{$\begin{array}{c}\text { Conclude } \\
\alpha=0.05\end{array}$} & \multirow{2}{*}{$\begin{array}{c}\text { Conclude } \\
\alpha=0.1\end{array}$} \\
\hline & Mean & SD & Mean & SD & & & & & & \\
\hline Case A & 0.76 & 0.61 & 3.29 & 3.57 & -2.71 & 15 & 1.75 & 1.34 & $\mathrm{H}_{\mathrm{A}}$ & $\mathrm{H}_{\mathrm{A}}$ \\
\hline Case B & 0.65 & 0.00 & 1.67 & 2.12 & -1.85 & 14 & 1.76 & 1.35 & $\mathrm{H}_{\mathrm{A}}$ & $\mathrm{H}_{\mathrm{A}}$ \\
\hline Case C & 4.98 & 0.19 & 3.80 & 1.84 & 2.49 & 15 & 1.75 & 1.34 & $\mathrm{H}_{\mathrm{A}}$ & $\mathrm{H}_{\mathrm{A}}$ \\
\hline Case D & 8.27 & 1.59 & 4.18 & 3.77 & 3.87 & 19 & 1.73 & 1.33 & $\mathrm{H}_{\mathrm{A}}$ & $\mathrm{H}_{\mathrm{A}}$ \\
\hline
\end{tabular}

Table F-2 T-tests Results for the Averages of |CP-90|

\begin{tabular}{|c|c|c|c|c|c|c|c|c|c|c|}
\hline & \multicolumn{2}{|c|}{ NLR } & \multicolumn{2}{|c|}{ Boot } & \multirow{2}{*}{$\begin{array}{l}\text { T- } \\
\text { stat }\end{array}$} & \multirow{2}{*}{ dof } & \multirow{2}{*}{$\begin{array}{l}\text { T-critical } \\
\alpha=0.05\end{array}$} & \multirow{2}{*}{$\begin{array}{c}\text { T-critical } \\
\alpha=0.1\end{array}$} & \multirow{2}{*}{$\begin{array}{l}\text { Conclude } \\
\alpha=0.05\end{array}$} & \multirow{2}{*}{$\begin{array}{c}\text { Conclude } \\
\alpha a=0.1\end{array}$} \\
\hline & Mean & SD & Mean & SD & & & & & & \\
\hline Case A & 7.87 & 1.43 & 6.91 & 8.48 & 0.43 & 15 & 1.75 & 1.34 & $\mathrm{H}_{0}$ & $\mathrm{H}_{0}$ \\
\hline Case B & 2.17 & 1.80 & 2.35 & 3.06 & -0.19 & 23 & 1.71 & 1.32 & $\mathrm{H}_{0}$ & $\mathrm{H}_{0}$ \\
\hline Case C & 3.38 & 0.28 & 7.09 & 2.60 & -5.50 & 15 & 1.75 & 1.34 & $\mathrm{H}_{\mathrm{A}}$ & $\mathrm{H}_{\mathrm{A}}$ \\
\hline Case D & 8.14 & 0.50 & 5.12 & 4.97 & 2.35 & 15 & 1.75 & 1.34 & $\mathrm{H}_{\mathrm{A}}$ & $\mathrm{H}_{\mathrm{A}}$ \\
\hline
\end{tabular}

